University of Nebraska - Lincoln

DigitalCommons@University of Nebraska - Lincoln

U.S. National Park Service Publications and

Papers

National Park Service

3-2021

\title{
Ecological Risk Assessment of Managed Relocation as a Climate Change Adaptation Strategy
}

\author{
Aviv Karasov-Olson \\ University of California, Davis \\ Mark W. Schwartz \\ University of California, Davis \\ Julian D. Olden \\ University of Washington - Seattle Campus \\ Sarah Skikne \\ Follow this and additional works at: https://digitalcommons.unl.edu/natlpark \\ University of Minnesota - Twin Cities \\ Part of the Atmospheric Sciences Commons, Climate Commons, Environmental Education Commons, \\ Jessica J. Hellmann \\ Environmentall lndicators and Impact Assessment Commons, Environmental Monitoring Commons, \\ University of Minnesota - Twin Cities \\ Environmental Policy Commons, Environmental Studies Commons, Fire Science and Firefighting \\ Commons, Leisure Studies Commons, Natural Resource Economics Commons, Natural Resources

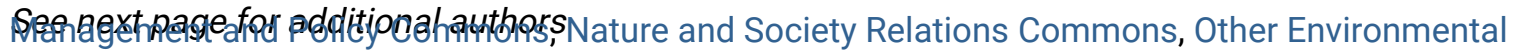 \\ Sciences Commons, Physical and Environmental Geography Commons, Public Administration Commons, \\ Recreation, Parks and Tourism Administration Commons, Science and Technology Policy Commons, and \\ the Science and Technology Studies Commons
}

Karasov-Olson, Aviv; Schwartz, Mark W.; Olden, Julian D.; Skikne, Sarah; Hellmann, Jessica J.; Allen, Sarah; Brigham, Christy; Buttke, Danielle; Lawrence, David J.; Miller-Rushing, Abraham J.; Morisette, Jeffrey T.; Schuurman, Gregor W.; Trammell, Melissa; and Hoffman, Cat Hawkins, "Ecological Risk Assessment of Managed Relocation as a Climate Change Adaptation Strategy" (2021). U.S. National Park Service Publications and Papers. 226.

https://digitalcommons.unl.edu/natlpark/226

This Article is brought to you for free and open access by the National Park Service at DigitalCommons@University of Nebraska - Lincoln. It has been accepted for inclusion in U.S. National Park Service Publications and Papers by an authorized administrator of DigitalCommons@University of Nebraska - Lincoln. 


\section{Authors}

Aviv Karasov-Olson, Mark W. Schwartz, Julian D. Olden, Sarah Skikne, Jessica J. Hellmann, Sarah Allen, Christy Brigham, Danielle Buttke, David J. Lawrence, Abraham J. Miller-Rushing, Jeffrey T. Morisette, Gregor W. Schuurman, Melissa Trammell, and Cat Hawkins Hoffman 
Service

U.S. Department of the Interior

\section{Ecological Risk Assessment of Managed Relocation as a Climate Change Adaptation Strategy}

Natural Resource Report NPS/NRSS/CCRP/NRR—2021/2241

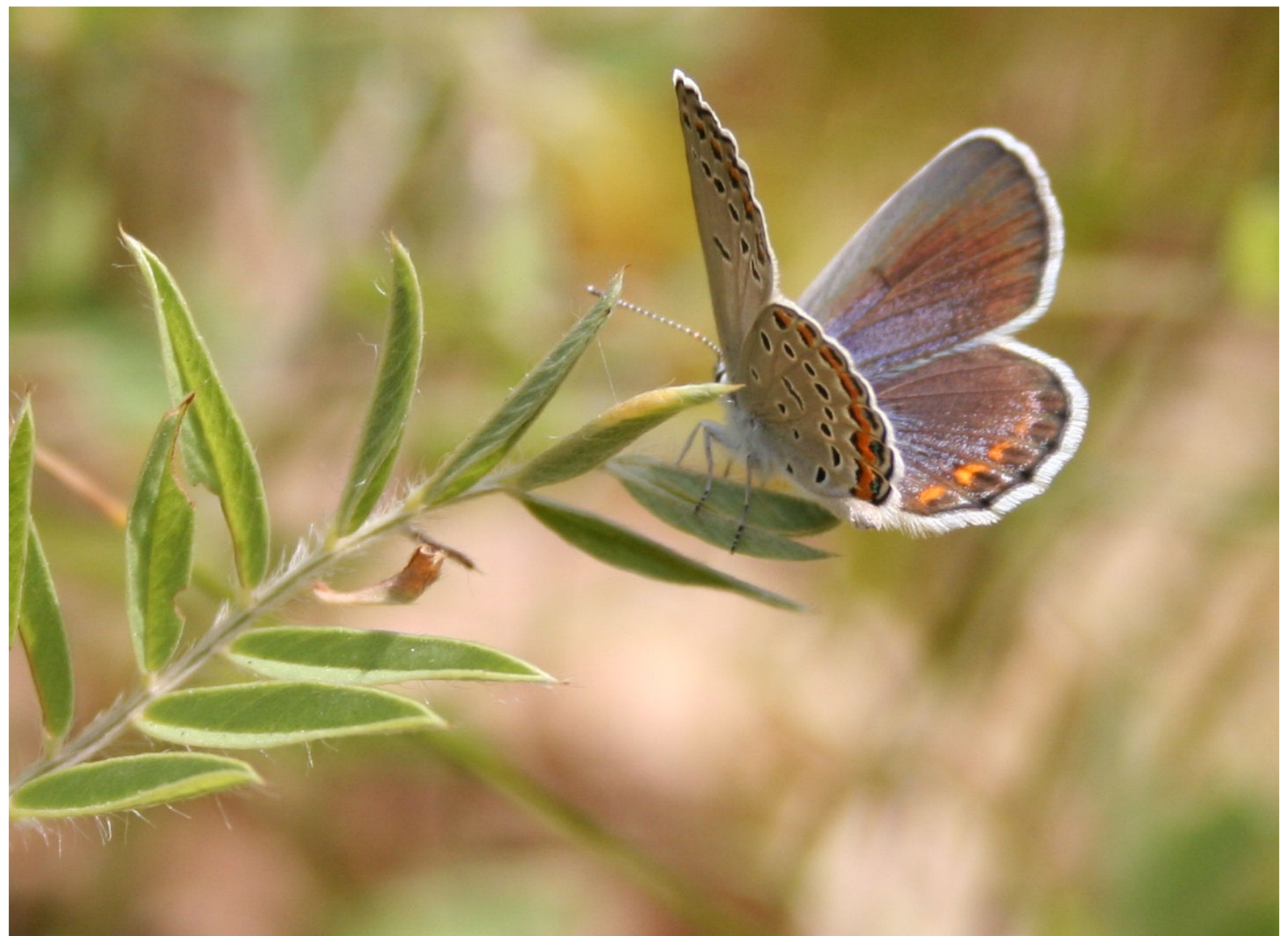


The production of this document cost $\$ 37,286$, including costs associated with data collection, processing, analysis, and subsequent authoring, editing, and publication.

\section{ON THE COVER}

Karner blue butterfly (Plebejus melissa samuelis)

Credit: NPS 


\section{Ecological Risk Assessment of Managed Relocation as a Climate Change Adaptation Strategy}

Natural Resource Report NPS/NRSS/CCRP/NRR—2021/2241

Aviv Karasov-Olson ${ }^{1}$, Mark W. Schwartz ${ }^{1}$, Julian D. Olden ${ }^{2}$, Sarah Skikne ${ }^{3}$, Jessica J. Hellmann ${ }^{3}$, Sarah Allen $^{4 \mathrm{a}}$, Christy Brigham ${ }^{4 \mathrm{~b}}$, Danielle Buttke ${ }^{4 \mathrm{c}}$, David J. Lawrence ${ }^{4 \mathrm{~d}}$, Abraham J. MillerRushing $^{4 \mathrm{e}}$, Jeffrey T. Morisette ${ }^{5}$, Gregor W. Schuurman ${ }^{4 \mathrm{~d}}$, Melissa Trammell ${ }^{4 \mathrm{f}}$, and Cat Hawkins Hoffman $^{4 \mathrm{~d}}$

${ }^{1}$ University of California, Davis, Department of Environmental Science and Policy, Davis, CA 95616

${ }^{2}$ School of Aquatic and Fishery Sciences, University of Washington, Seattle, WA 98105

${ }^{3}$ Institute on the Environment, University of Minnesota, Saint Paul, MN 55108

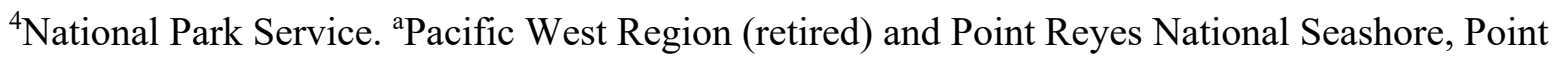
Reyes Station, CA 94956; 'Sequoia and Kings Canyon National Parks, Three Rivers, CA 93633; ${ }^{\mathrm{c}}$ Biological Resources Division, Fort Collins, CO 80525; ${ }^{\mathrm{d} C l i m a t e}$ Change Response Program, Fort Collins, CO 80525; ${ }^{\mathrm{e} A c a d i a}$ National Park, Bar Harbor, ME 04609; ${ }^{\mathrm{D} D O I}$ Regions 6/7/8, Grand Junction, CO 81506

${ }^{5}$ U.S. Department of the Interior, National Invasive Species Council, Fort Collins, CO 80524

March 2021

U.S. Department of the Interior

National Park Service

Natural Resource Stewardship and Science

Fort Collins, Colorado 
The National Park Service, Natural Resource Stewardship and Science office in Fort Collins, Colorado, publishes a range of reports that address natural resource topics. These reports are of interest and applicability to a broad audience in the National Park Service and others in natural resource management, including scientists, conservation and environmental constituencies, and the public.

The Natural Resource Report Series is used to disseminate comprehensive information and analysis about natural resources and related topics concerning lands managed by the National Park Service. The series supports the advancement of science, informed decision-making, and the achievement of the National Park Service mission. The series also provides a forum for presenting more lengthy results that may not be accepted by publications with page limitations.

All manuscripts in the series receive the appropriate level of peer review to ensure that the information is scientifically credible, technically accurate, appropriately written for the intended audience, and designed and published in a professional manner.

This report received formal peer review by subject-matter experts who were not directly involved in the collection, analysis, or reporting of the data, and whose background and expertise put them on par technically and scientifically with the authors of the information.

Views, statements, findings, conclusions, recommendations, and data in this report do not necessarily reflect views and policies of the National Park Service, U.S. Department of the Interior. Mention of trade names or commercial products does not constitute endorsement or recommendation for use by the U.S. Government.

This report is available in digital format from the Natural Resource Publications Management website. If you have difficulty accessing information in this publication, particularly if using assistive technology, please email irma@nps.gov.

Please cite this publication as:

Karasov-Olson, A., M. W. Schwartz, J. D. Olden, S. Skikne, J. J. Hellmann, S. Allen, C. Brigham, D. Buttke, D. J. Lawrence, A. J. Miller-Rushing, J. T. Morisette, G. W. Schuurman, M. Trammell, and C. Hawkins Hoffman. 2021. Ecological risk assessment of managed relocation as a climate change adaptation strategy. Natural Resource Report NPS/NRSS/CCRP/NRR—2021/2241. National Park Service, Fort Collins, Colorado. https://doi.org/10.36967/nrr-2284919.

NPS 999/175141, March 2021 


\section{Contents}

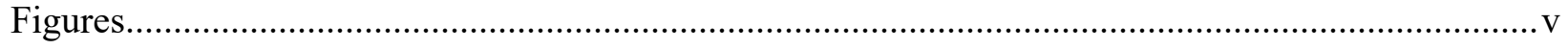

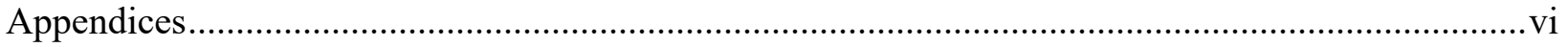

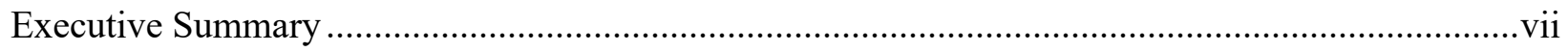

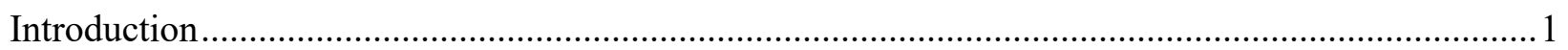

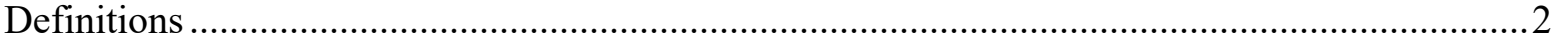

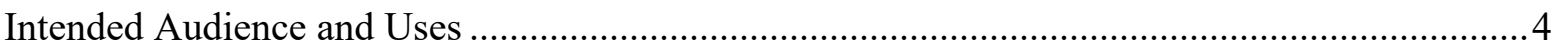

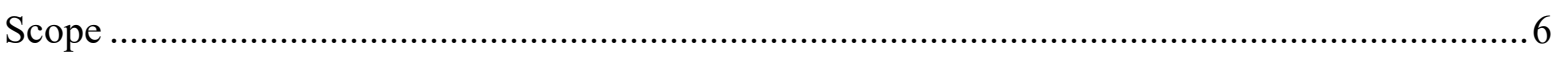

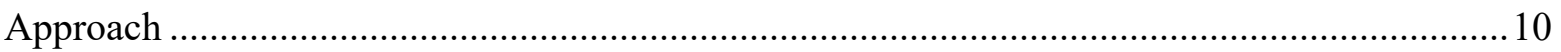

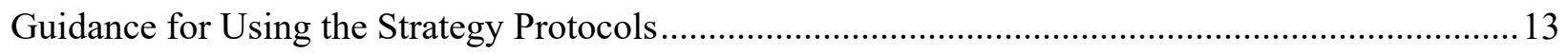

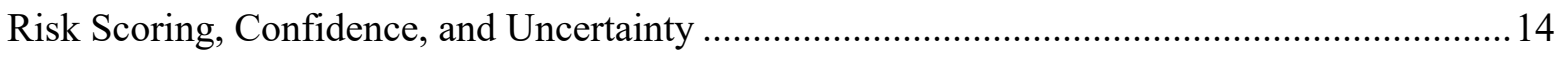

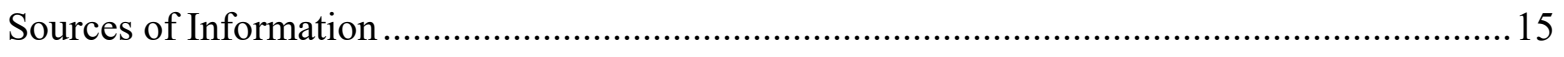

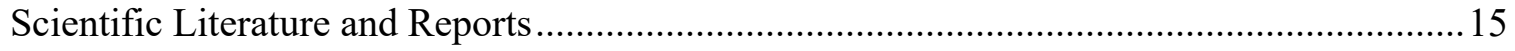

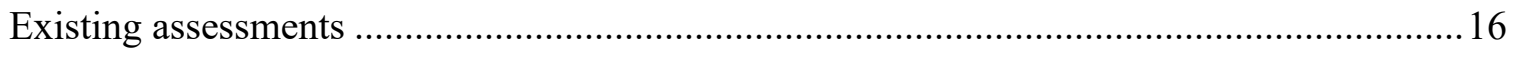

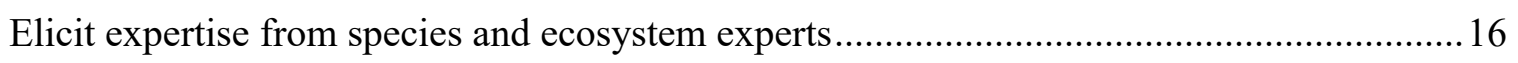

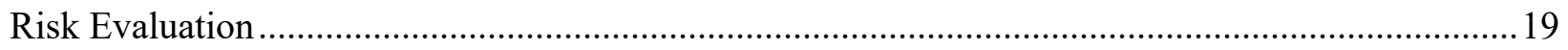

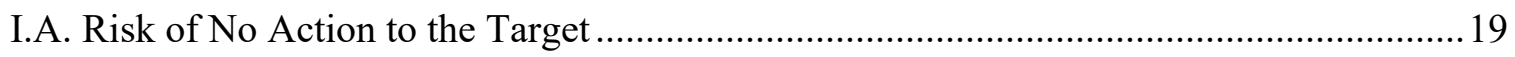

I.B. Risk of No Action to the Recipient Ecosystem ....................................................... 21

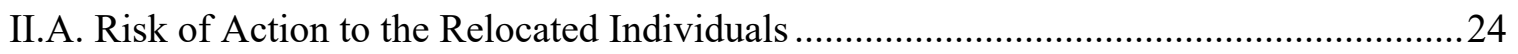

II.B. Risk That the Target Source Population Cannot Withstand Diminished

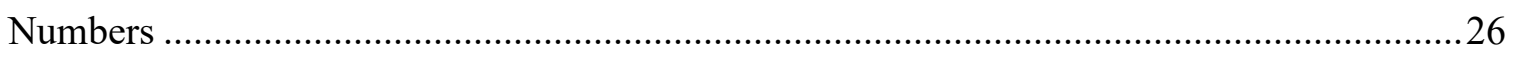

II.C. Risk That Removing Individuals of the Target Will Negatively Impact a

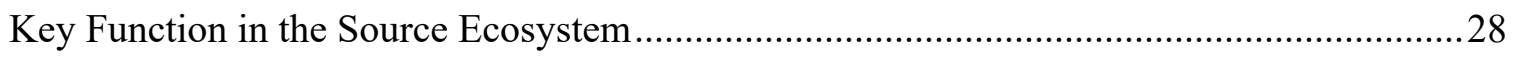

II.D. Risk of Causing Undesired Evolution in the Target ..................................................29

III.A. Risk of Target Transmitting Novel Disease or Associated Pest..................................31

III.B. Risk of Competitive Interaction Negatively Affecting the Distribution or

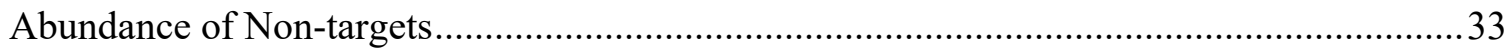




\section{Contents (continued)}

III.C. Risk of Consumptive Effects Reducing the Abundance or Distribution of Non-targets

III.D. Risk of Driving Undesired Evolution in Non-targets ..................................................35

IV.A. Risk of Indirect and Negative Impacts on Ecosystem Structure …..............................37

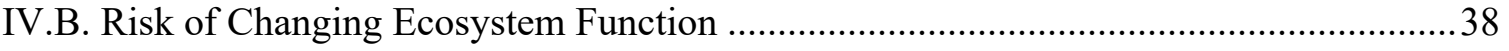

V.A. Risk of Invasion Within the Intended Recipient Ecosystem ....................................41

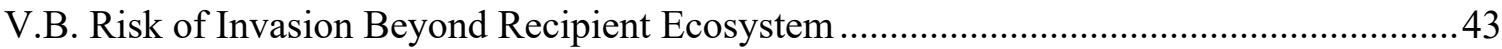

V.C. Risk of Irreversibility of The Managed Relocation Action ..........................................44

VI.A. Risk to a Culturally or Economically Important Species .........................................47

VI.B. Risk to a Valued Ecosystem Service .................................................................... 48

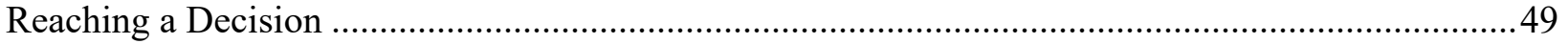

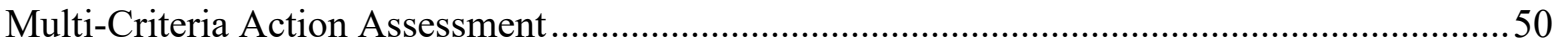

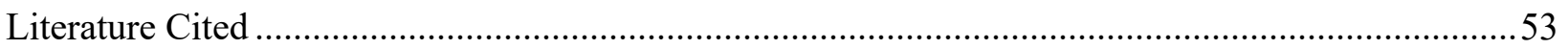




\section{Figures}

Page

Figure 1. Climate smart conservation cycle modified from Stein et al. (2014). .............................. 7

Figure 2. Five critical questions that must be evaluated before making a managed relocation decision.

Figure 3. Screen capture of the managed relocation risk assessment tool presented in a simple spreadsheet format. 13

Figure 4. Confidence is a function of the uncertainty that comes from the amount, relevance, and quality of evidence, and whether the available evidence is in agreement (IPCC 2013).

Figure 5. Screen capture of Section I of the managed relocation risk assessment spreadsheet presented in a simple spreadsheet format.

Figure 6. Screen capture of Section II of the managed relocation risk assessment spreadsheet presented in a simple spreadsheet format.

Figure 7. Screen capture of Section III of the managed relocation risk assessment protocol presented in a simple spreadsheet format.

Figure 8. Screen capture of Section IV of the managed relocation risk assessment protocol presented in a simple spreadsheet format.

Figure 9. Screen capture of Section V of the managed relocation risk assessment protocol presented in a simple spreadsheet format.

Figure 10. Screen capture of Section VI of the managed relocation risk assessment protocol presented in a simple spreadsheet format.

Figure 11. Schematic table of a visual risk summary where 17 boxes represent the individual risk assessments within the six risk sections (rows) and specific criteria as described above (columns). Boxes are color coded for assessed risk from green (low risk) to red (very high risk) 


\section{Tables}

Table 1. An outline of the expert elicitation process based on Hemming et al. (2018).................... 17

Table 2. Representative characteristics of many invasive species (Baker 1965; Roy 1990;

Sakai et al. 2001; Marchetti et al. 2004; Hayes and Barry 2008) and how those traits may be related to invasion (modified from Olden et al. 2011) ............................................................... 42

Table 3. Representative (hypothetical) decision issues that could be used to create alternative action scenarios for a managed relocation project.

\section{Appendices}

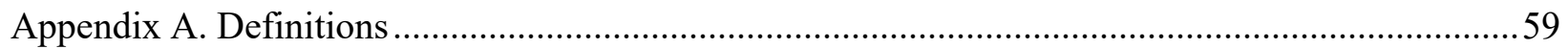

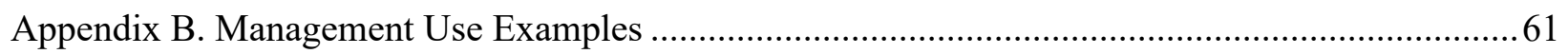

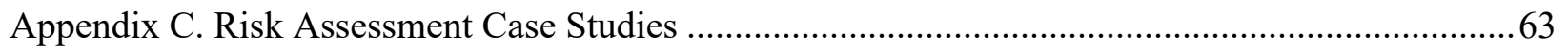




\section{Executive Summary}

Changing climate and introduced species are placing an increasing number of species at risk of extinction. Increasing extinction risk is increasing calls to protect species by relocating, or translocating, them to locations with more favorable biotic or climatic conditions. Managed relocation, or assisted migration, of species entails risks to both the conservation target organisms being moved as well as the recipient ecosystems into which they are moved.

Recognizing this risk, calls have been made for practitioners interested in considering a managed relocation project to engage in a serious risk assessment prior to advancing a project. We engaged a team of researchers and resource managers to create risk assessment protocols that could be used by natural resource managers within U.S. National Parks, or elsewhere, to help inform a decision of whether the risks involved in managed relocation are warranted. These protocols facilitate evaluation of the ecological risk of species managed relocation as part of planning and decision making. This is not a policy document. It neither introduces new policy, nor serves to interpret or resolve current policies regarding managed relocation (or assisted migration) as a natural resource management strategy.

We assembled a team of five university researchers and ten federal resource management researchers and staff to develop a practical management-oriented risk assessment strategy. We jointly agreed to a set of principles to guide this managed relocation risk assessment strategy. This protocol and accompanying spreadsheet would be used to help a decision-maker structure a decision process but would not strive to provide a formulaic decision output. Identifying, evaluating, and managing risk is a subjective decision that is the responsibility of the decision authority.

We began by defining the scope of this work to include moving populations or species for the purpose of conserving the target populations or species that are threatened by climate or invasive species. We also included species movements for the purpose of retaining some critical ecosystem function. We did not include management actions such as planned ecosystem re-alignment for climate change or other kinds of translocations associated with ecosystem manipulation (e.g., habitat restoration), although these protocols may be useful for some of those management actions with minor modification.

We adopted the premise that risk decisions are inherently subjective and that different aspects of risk (e.g., the risk of a moved species introducing a novel pathogen to an ecosystem, the risk of unwanted evolution in the moved species) are non-additive. Hence, our strategy is designed to encourage managers to think broadly and comprehensively about risk in order to make the best possible decision given alternate opposing risks (i.e., the risk of extinction versus the risk of causing unintended harm to other species and ecosystems in the process of trying to save a species).

We identified six major areas of risk, with a total of seventeen sub-categories. These are:

- Risks of no managed relocation action. Risk of:

$\circ$ no action on the target 
○ no action on the recipient ecosystem

- Risks of managed relocation action to the target. Risks of:

$\circ$ action on the translocated individuals

○ target source population extirpation through diminished numbers

$\circ$ reduced ecological functioning of the source ecosystem

○ causing undesired evolution in the target

- Risks of action on non-targets in the recipient ecosystem. Risks of:

o target transmitting novel disease or associated pest

$\circ$ negative competitive interactions on non-target populations

$\bigcirc$ predation, herbivory, or allelopathic effects on non-target populations

$\circ$ driving undesirable evolution in non-target species

- Risks of action on higher order attributes of the recipient ecosystem. Risks of:

$\circ$ indirect and negative impacts on ecosystem structure

$\circ$ changing ecosystem function

- Risks associated with invasion. Risks of:

○ invasion within the intended recipient ecosystem

o invasion beyond recipient ecosystem

$\circ$ irreversibility of the managed relocation action

- Risks associated with socio-economic values. Risks to:

○ culturally or economically important species

○ valued ecosystem services

For each risk category we provide guidance on risk scoring. Risk scoring is comprised of a risk rank category (low, moderate, high, very high) and a confidence score (low, medium, high). Confidence is a combined attribute of the strength of evidence and the agreement of that evidence.

The protocols are presented in an accompanying Excel spreadsheet that uses a graphical tool to allow users to visualize a composite of risk and confidence. We are adamant about not summing across risk categories. Instead, we provide a graphing tool that summarizes risk within categories. We suggest that users could find risks posed by a proposed action to be acceptable if:

1) Confidence scores are sufficient that managers feel confident that the risk assessment is informative;

2) There is no single risk category that is so high and so important as to make the project unacceptably risky; and

3) The general distribution of risk is not so high as to exceed some level of expectation that one of many potential problems could arise and lead to decision regret.

We frame this risk assessment within the context of other critical questions that need to be answered in order to proceed toward strategic planning for a managed relocation action. These include justifying ecological need, assessing technical feasibility, cost, management priority and social 
acceptability. If all these criteria are met, then these same protocols can be used in a multi-criteria assessment to compare across different strategic plans for managed relocation (e.g., relocation location, relocation numbers, source and husbandry of relocated individuals). We provide brief guidance on how that may be completed with no presumption of final decision determination.

Finally, in the process of developing these risk scoring protocols, we tested them on a suite of four case studies (bull trout, Karner blue butterfly, giant sequoia and Pitcher's thistle). These are provided in this document as examples of the logic and process that we outline for assessing risk. These were, however, done without broad consultation and should be taken not as definitive risk assessments of managed relocation for these species, but as examples of how one might use our strategy for an assessment of ecological risk associated with managed relocation.

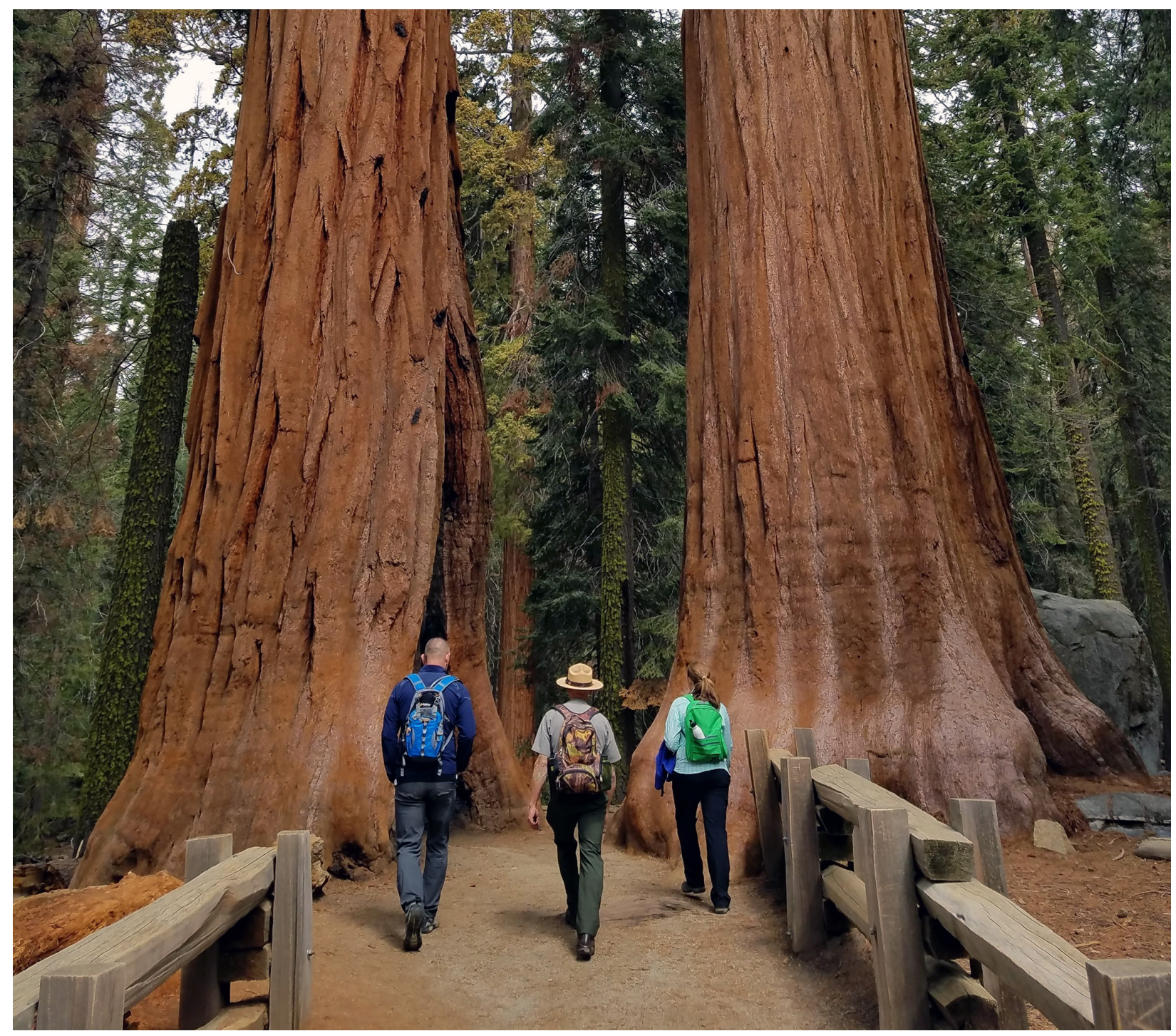

Giant sequoia trees line a trail in Giant Forest in Sequoia National Park. Credit: M. Holly, NPS 


\section{Introduction}

Nature is complex and we operate from a position of never having as much information as we would like. Nevertheless, evidence that species may go extinct as a consequence of environmental change is compelling (UNEP 2019). Consequently, managers are increasingly considering actions such as managed relocation with the hope to save components of nature, even if such actions present risks of the species becoming invasive or other unforeseen dynamics of species introductions (Kostyack et al. 2011; Lawler and Olden 2011; Wallingford et al. 2020). Indeed, lessons can be learned from species introduced under the auspice of biocontrol, where more cautious and transparent risk analysis might have avoided the challenges and controversies of the early efforts (Barratt et al. 2010, Barratt et al. 2018). Understanding the nature of risks involved should be a key step towards decision-making in all relocation actions (ISAC 2017).

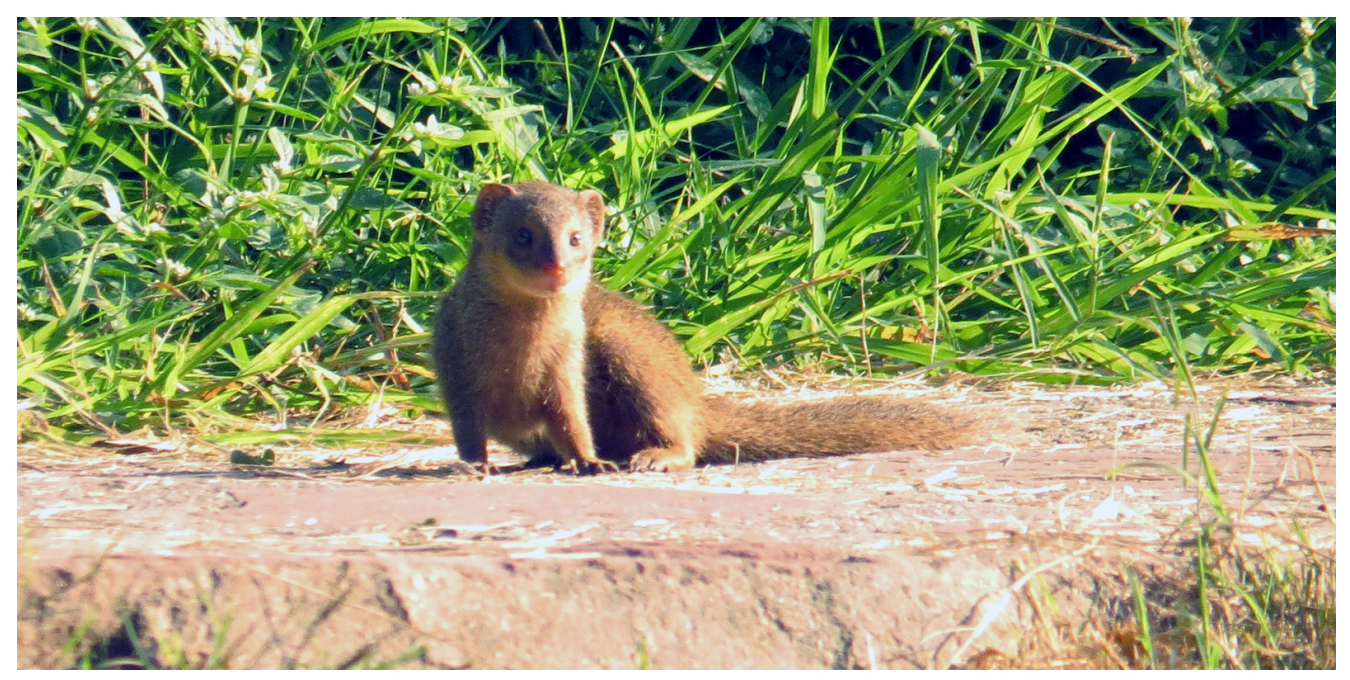

The small Indian mongoose (Herpestes javanicus auropunctatus) was introduced to Hawai'i by the sugar industry to control rats in sugarcane fields but instead decimated native wildlife population including several threatened and endangered birds (Resnik 2018). Credit: D. Hobern, CCA 2.0

These protocols are tools to guide evaluation of the
ecological risk of species managed relocation as part of
planning and decision making. The protocols do not
address existing, or proposed, policies regarding
managed relocation (or assisted migration) as a natural
resource management strategy.

These protocols provide guidance for qualitative ecological risk assessment to inform management decisions regarding proposed movements of populations or species beyond their historical range as a conservation management response to climate change or invasive species. The protocols provided here are not designed to answer the question "Should management move a species?" This subjective question must be answered by the decision-maker. Protocol completion informs that decision by 
formalizing a risk analysis and risk characterization. The purpose of assessing risk is not to preclude such actions by promoting an environment of risk aversion. Rather, these protocols inform risk management by assisting the user in evaluating the risk of causing harm by either taking or not taking a managed relocation action and balancing this risk against perceived benefits of the proposed action.

Managed relocation efforts will likely never be $100 \%$ successful, and such efforts face the same risk that intensive (and often costly) restoration efforts faced in the past - namely that resources may be expended without providing a positive return on the investment (Ricciardi and Simberloff 2009; Schwartz et al 2012). Therefore, it is important to evaluate risks of action and inaction early in the decision-making process. This strategy does not point to a decision but is instead intended to contribute to scientifically informed decisions through a considered risk evaluation for all options.

\section{Definitions}

Managed relocation has been defined as moving individuals of a species beyond the species' historical distribution for the purpose of conservation under climate change (McLachlan et al 2007; Richardson et al. 2009). We include management actions to reduce threats driven by invasive species as well. We refer to the focal species to be relocated as the 'target' of the managed relocation action. The protocols we describe primarily center on species, however, we use the term 'target' to also encompass sub-species, populations, or genotypes as potential targets of managed relocation (see Appendix A for definitions). We treat the term 'managed relocation' as similar to and encompassing 'assisted migration', 'assisted dispersal', and 'ecological replacement,' and as a subset of the broader set of actions known as 'conservation translocations' (McCormack 2018). Conservation translocations include actions such as reinforcing existing populations within a target's historical range as well as re-establishing historical populations. As an example, restoring wolves to Yellowstone would constitute a conservation translocation, not a managed relocation, given that this was restoring populations lost due to human extirpation. An example such as the wolf could be considered a managed relocation if the individuals moved are of specific genotypes used to help establish populations that were more likely to persist under changed climates. 


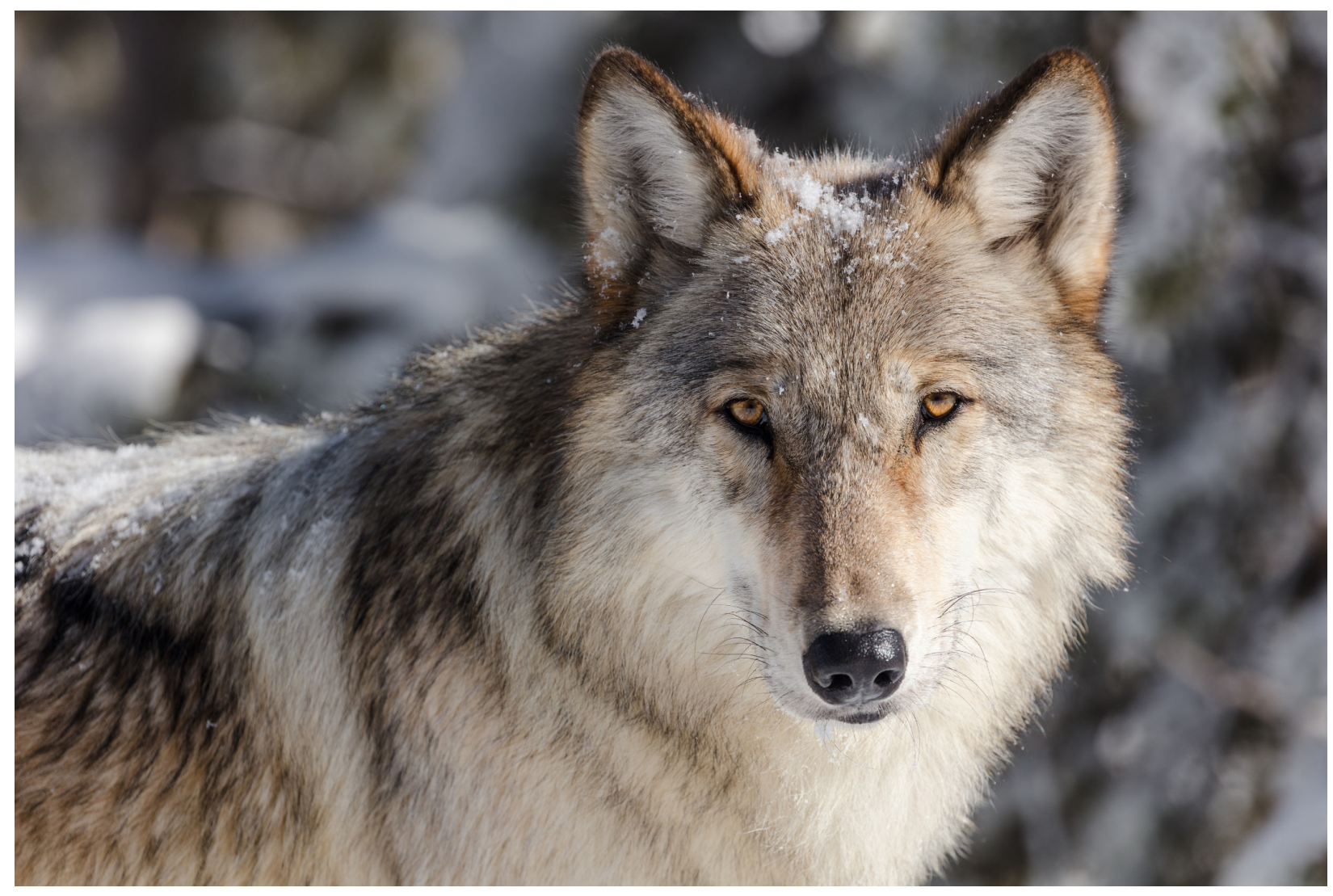

Wolves were restored to Yellowstone in 1995. The population has since recovered substantially and become a popular visitor feature of the park. Credit: J. Frank, NPS

These protocols are designed to facilitate a systematic review of the potential risks associated with managed relocation. The EPA (1998) defines an ecological risk assessment as one that "evaluates the likelihood that adverse ecological effects may occur or are occurring as a result of exposure to one or more stressors." These protocols frame managed relocation as the stressor. The EPA further divides an ecological risk assessment into three phases: problem formation, analysis, and risk characterization:

In problem formulation, risk assessors evaluate goals and select assessment endpoints, prepare the conceptual model, and develop an analysis plan. During the analysis phase, assessors evaluate exposure to stressors and the relationship between stressor levels and ecological effects. In the third phase, risk characterization, assessors estimate risk through integration of exposure and stressor-response profiles, describe risk by discussing lines of evidence and determining ecological adversity, and prepare a report. The interface among risk assessors, risk managers, and interested parties during planning at the beginning and communication of risk at the end of the risk assessment is critical to ensure that the results of the assessment can be used to support a management decision. Because of the diverse expertise required (especially in complex ecological risk assessments), risk assessors and risk managers frequently work in multidisciplinary teams. (EPA 1998) 
We generally follow this definition of ecological risk assessment, emphasizing the analysis and risk characterization phases of the process, assuming that a problem formulation precedes understanding a need for a risk characterization.

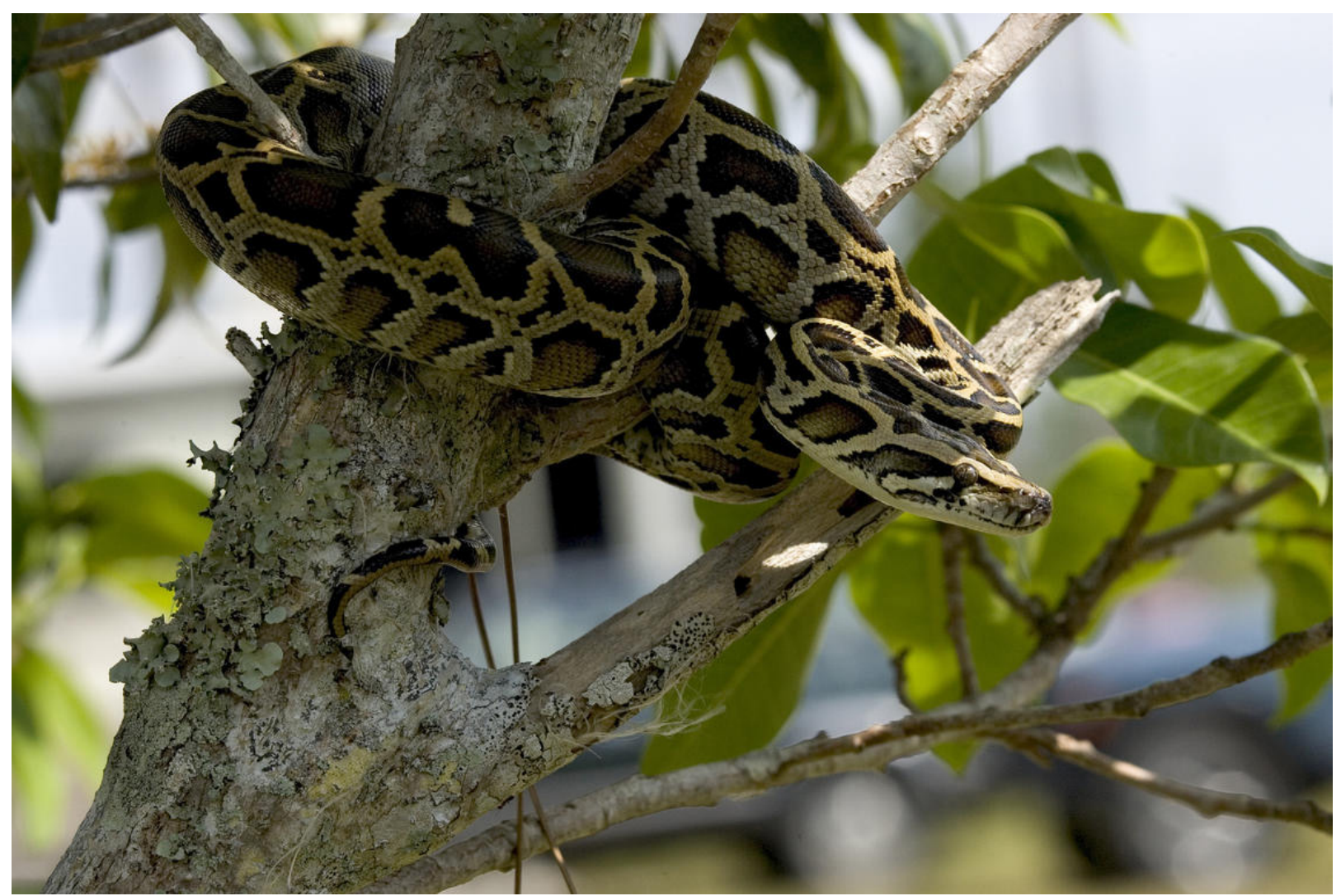

Ecological risk assessment based on history of establishment, suitability, and natural history traits has shown that the importation of many species for the pet trade, including the Burmese python (Python bivittatus) shown above, pose threats to native species. The Burmese python is highlighted because large constrictors pose a particularly high risk of becoming invasive (Reed and Rodda 2009). Credit: R. Cammauf, NPS

\section{Intended Audience and Uses}

The intended audiences for this risk assessment strategy are biologists, ecologists, and managers responsible for decisions regarding managed relocations involving natural areas, such as federal and state parks and protected areas. We envision two primary scenarios in which readers can use these protocols to consider managed relocation. First, we address cases where the historical range of a target becomes unsuitable, and the target might not be able to disperse on its own to newly suitable sites. Here, the intended use is for considering managed relocations in response to threats to targets where climate change, at least in part, increases extirpation risk. Second, we seek to inform cases where changing environmental conditions are compromising an existing ecosystem. In this case the intended use is for considering managed relocations aiming to recover or retain ecosystem functionality in the recipient ecosystem, to foster longer term conservation goals in light of the dynamics on the landscape (e.g., ecological replacement). 
Alternative applications include consideration of conservation translocations that release species within their historical range (e.g., conservation repatriations, population restorations) either for their benefit or because they provide a beneficial ecosystem function. Because some attributes of risk are in common, these protocols can inform a risk evaluation for such actions. However, we generally do not see the same need to evaluate risks when restoring species to sites where they historically occurred, because such restorations would generally include attributes associated with low ecological risk. Other applications include cases where other stressors, such as invasive species, pollution, disease, or development ${ }^{1}$ adjacent to a target area render the historical range of a species unusable and moving the species beyond the historical range is considered in order to avoid extirpation.

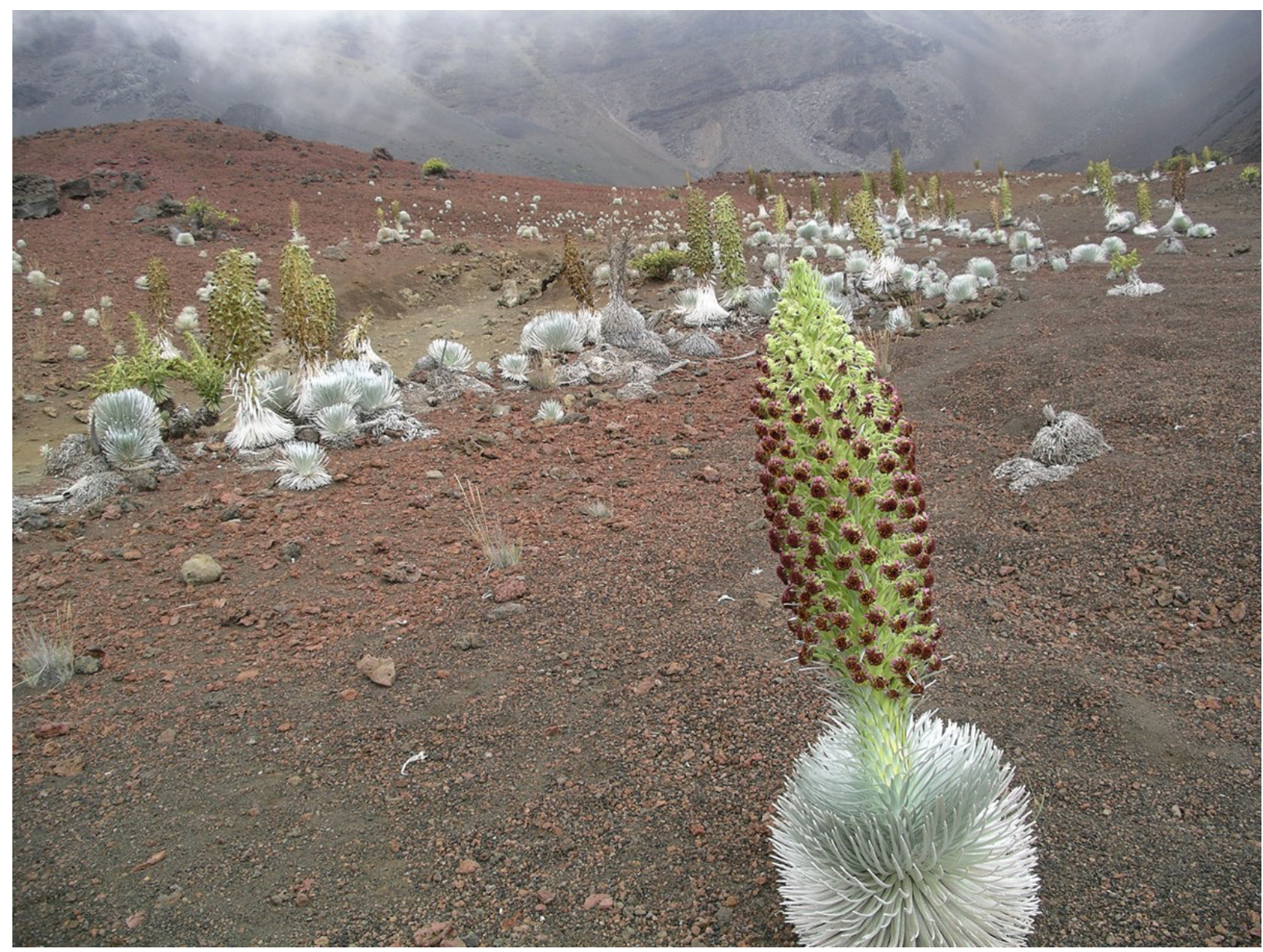

Haleakalā silversword (Argyroxiphium sandwicense subsp. Macrocephalum) in Haleakalā National Park is endangered due to over-browsing by ungulates. Outplanting is one strategy that has been used to help recover this population (Walker and Powell 1999). Credit: NPS

These protocols and accompanying spreadsheet may be used for these other applications, although planning for such action(s) may be very different among these examples; assessing risks of

\footnotetext{
${ }^{1}$ See Salafsky et al. 2008 and IUCN 2017a for a more complete list of potential threats to species extinction.
} 
conservation translocations in response to stressors other than climate and invasive species is not the impetus for which these protocols were developed. Finally, these protocols focus on moving a single target; it does not attempt to assess the risk of relocating multiple taxa (e.g., for the purposes of transforming the recipient system). Hence, users should be vigilant for potential gaps in risk assessment that derive from using this assessment for objectives other than our intent: assessing ecological risks associated with proposals to move a single target beyond its historical range, as a means of conservation in response to climate change or invasive species.

We presume that the most common usage will be for proposals to move a target into or within managed protected areas, or between protected areas through agreements or other authorities. We suggest this approach is useful for managers to undertake irrespective of whether the source population, the target destination, or both fall fully within their jurisdiction.

\section{Scope}

These risk assessment protocols represent an important but narrowly focused component within a larger planning framework for natural resource management. The NPS, as an example, uses adaptive management to guide planning (Williams et al. 2009), and one particularly relevant form of Adaptive Management planning is the Climate Smart Adaptation Guide (Stein et al. 2014, Fig 1). These protocols can be used within this planning process and presumes that a resource manager has completed preliminary climate adaptation steps of defining the planning purpose and scope (step 1), assessing climate impacts and vulnerabilities (step 2), reviewing and revising conservation goals and objectives (step 3), and identifying managed relocation as a possible adaptation option (step 4). The protocol spreadsheet is a tool to assist in evaluating options (step 5 - evaluate and select options), specifically managed relocation.

Additionally, the IUCN Guidelines for Reintroductions (IUCN/SSC 2013), sections 3-5, provide guidance to assist in this preliminary planning of species translocation. However, the risk assessment (section 6) of the IUCN document describes attributes of risk but does not provide instructions for evaluation of that risk. Providing step-by-step guided protocols to evaluate risk is the objective of this document.

To apply these protocols, users should have at least a general sense of the projected benefit of taking the proposed managed relocation action. Further, the planning team should have a clear idea of the location(s) where they are considering releasing individuals of the target. Thus, we assume that preliminary actions that would call for managed relocation as a potential climate change adaptation action have been completed. With this important information in hand, the protocols evaluate the potential ecological risks of taking the proposed managed relocation action. If the reader remains uncertain about whether these protocols are relevant for a particular project, please see Appendix B for a suite of examples that describe management actions that do and do not fit within the scope covered here.

These risk assessment protocols have a narrow scope with respect to planning and decision making. First and foremost, the protocols are neutral to existing policies and make no assumptions regarding the capacity of an agency or organization to engage in any particular action. Second, management 
decisions must consider the multi-faceted benefits that could be derived from moving an organism (e.g., improved ecosystem function, ecological redundancy, restored food webs). Third, there are other factors that are likely to be considered for strategic implementation. For example, managers might consider gradual introductions of the target to evaluate success progressively. Finally, ecological risk will vary with a project's capacity to monitor outcomes and track specific details (genetic attributes of moved individuals; Barratt et al. 2010). Strategic implementation will have an impact on ecological risk. Thus, we urge users to continue to use these protocols after an initial decision as a tool to evaluate strategies that are important in minimizing risk during implementation.

The protocols presented here assume that assessment of the potential benefits of taking the action began earlier in the overall adaptation process, and that consideration of the potential benefits of a variety of alternative actions has fed into an overall process of evaluating and selecting actions (Figure 1, Step 5). Within this planning stage, we offer these risk protocols to answer just one among five critical questions (what is the ecological risk of managed relocation) that must be answered in the affirmative (Figure 2) before choosing to engage in managed relocation.

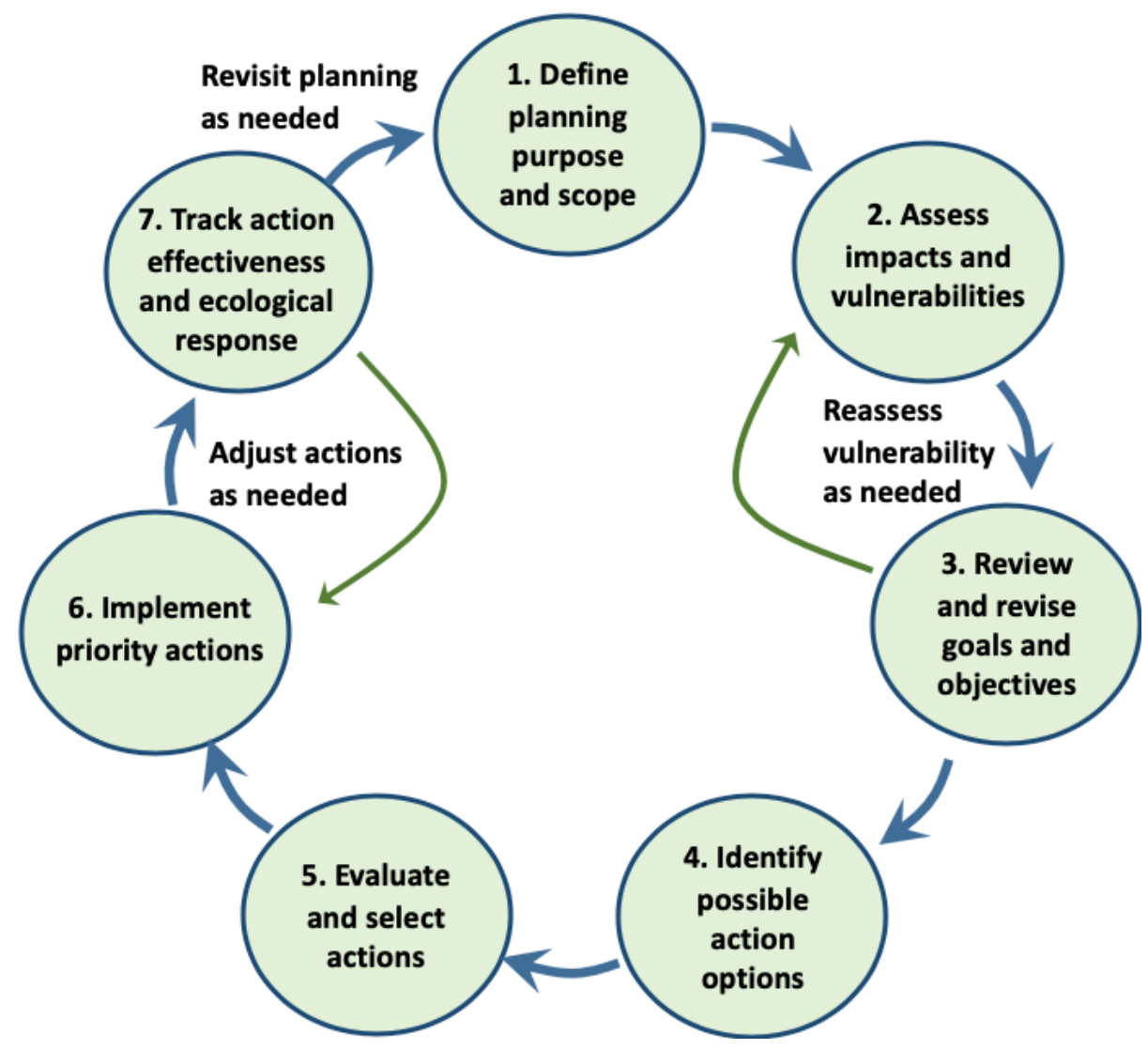

Figure 1. Climate smart conservation cycle modified from Stein et al. (2014). 


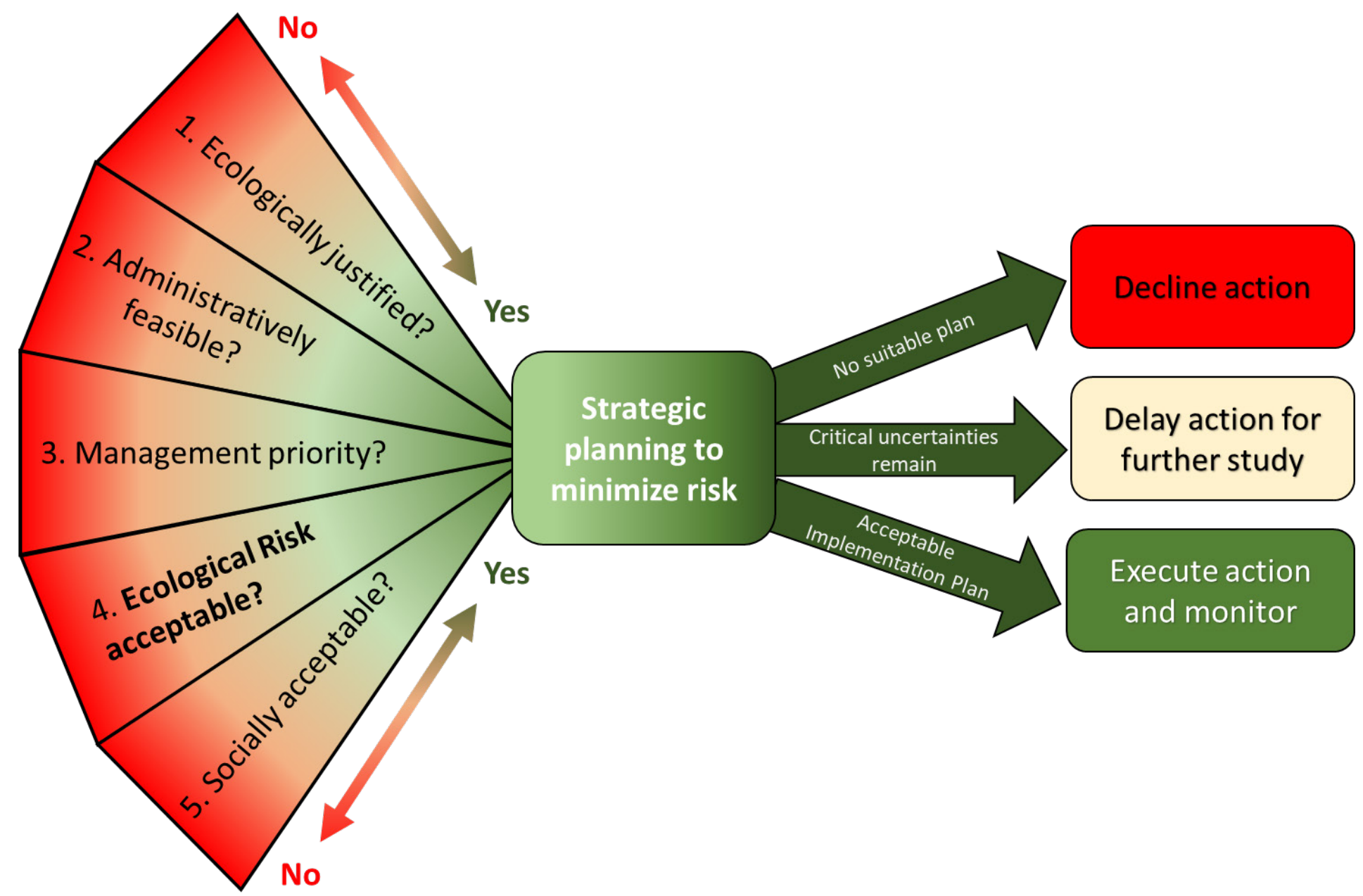

Figure 2. Five critical questions that must be evaluated before making a managed relocation decision. Questions that must be answered include whether the proposed action is ecologically justified, administratively feasible and a management priority. Then a risk assessment, as detailed here (question 4) can be completed. All along the process, managers must also assess whether or not the action would be socially acceptable. Satisfying these criteria, a manager may then consider minimizing risk among various specific options (e.g. how many individuals, from which populations to move, where to move them). Triangular wedges grading from red (no) to green (yes) indicate that these questions may be addressed at any time during the process, and that revisiting questions as conditions change is likely. 
The risk assessment strategy is divided into several sections. For example, we include a section that allows users to assess the risk to the target of no managed relocation and risk to the recipient ecosystem of no intervention, depending on the intended purpose of the action. In addition, a proposed action of moving a species for the benefit of the recipient ecosystem is a limited case under the broader realm of management actions to help transition ecosystems under global change. We also consider this potential action. However, there are attributes of risk that might be scored differently under different management objectives. For example, one might move a species into an ecosystem with the intent of accelerating ongoing regional ecosystem change. In this case, one might consider the introduction a failure if the moved species did not spread from the site into which it was introduced. Alternatively, a species might be introduced into a relatively isolated ecosystem with the hope of recovering or improving ecosystem functioning, with no intention of further spread. In this latter case, the risk of unintended secondary expansion and spread of a moved target would be considered a risk to the greater suite of ecosystems around the recipient ecosystem(s). These two scenarios would use a different risk assessment scoring for the likelihood of spread of the moved target. We present this dichotomy in order to caution users to be aware that we focused on a narrow set of potential management circumstances for these protocols. However, the protocols are adaptable and can be generalized to accommodate a wide variety of conditions, given particular management circumstances.

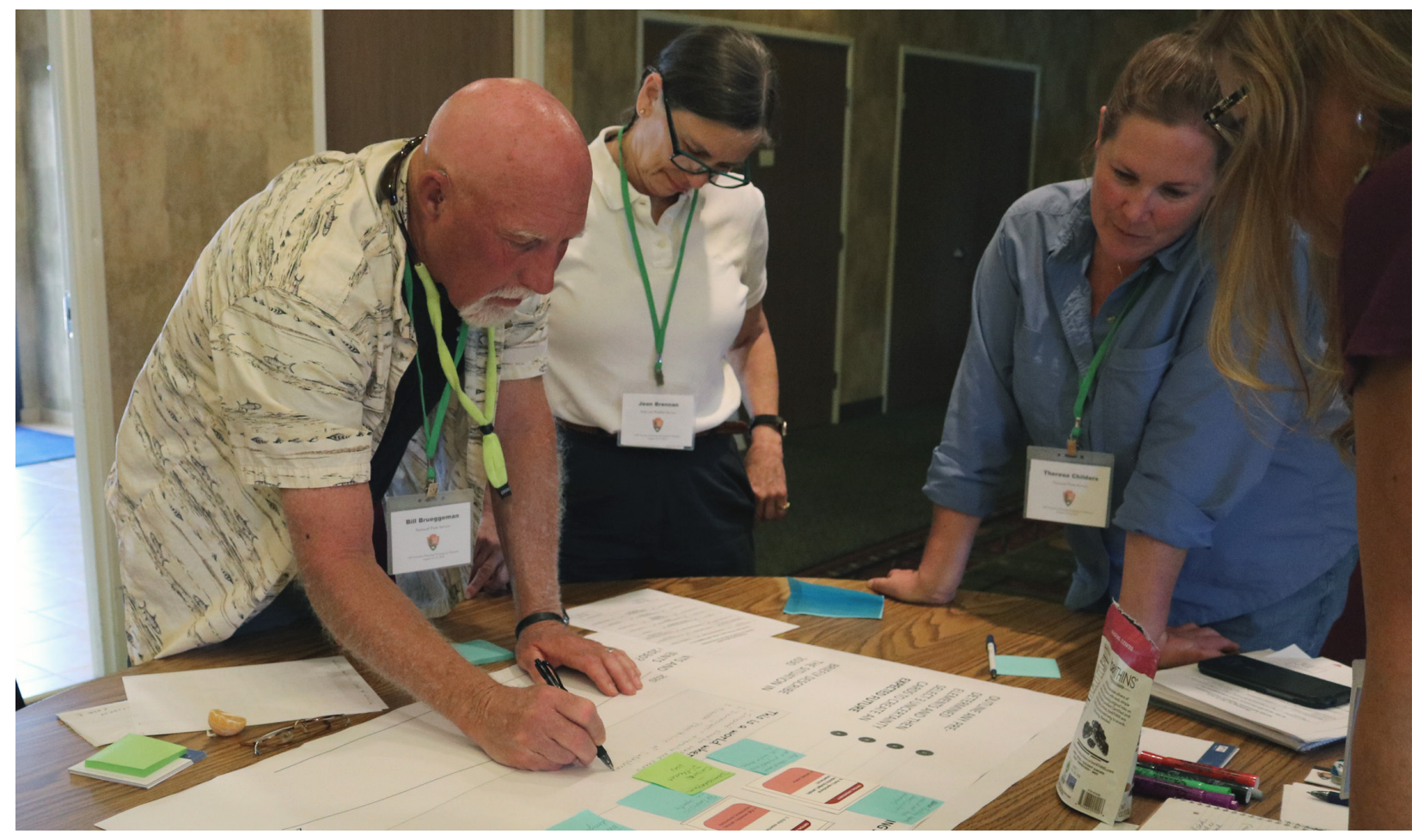

Participants at work in a scenario planning session to address climate change adaptation in Black Canyon of the Gunnison National Park. Resource management and other decisions in national parks can be a challenging process of balancing scientific understanding, public sentiment and values, and logistical feasibility. Credit: Larry Perez, NPS 
Managed relocation may also incur a risk of social acceptability of the action. Focusing primarily on ecological risk, these protocols only address limited aspects of social, ethical, and economic costs of taking a managed relocation action. For example, moving a species to protect it may alarm constituents who view the action as degrading the natural integrity of a park or other natural area. We include a brief section at the end that encourages users to carefully consider social values and how these may increase the risk of moving a species. There could indeed be significant and complex social issues involved with managed relocation (Richardson et al. 2009; Schwartz et al. 2012). Despite noting the need to assess social benefits and risks, our primary focus is on ecological risk. Following this ecological risk assessment there is likely to be a need for an assessment of social benefits and risks as part of the broader decision analysis. In that context, we suggest the results of this risk assessment may be beneficial to inform a stakeholder-engaged process to evaluate social willingness to absorb the cost, benefit, and ecological risk of a proposed managed relocation.

\section{Approach}

There are a variety of approaches to ecological risk assessment (Burgman 2009, Suter 2016). Specific recommendations for assessing ecological risk are particularly well-developed on the issues of releasing genetically modified organisms (e.g., Conner et al. 2003) and in biocontrol agents (e.g., Roy et al. 2017). Further, the Invasive Species Advisory Committee made recommendations for risk assessment related to managed relocation (ISAC 2017). We reviewed these literatures to settle on a broad, efficient, qualitative strategy to assess ecological risk on the decision of whether or not to engage in managed relocation.

The results of risk assessments conducted through this strategy can inform the comprehensive analyses necessary for decisions. We do not advocate avoiding all risk at all costs; this is not realistic. When facing two or more potentially negative outcomes, it is best to evaluate the risks of competing actions and realistically manage the risk associated with the ultimate management decision. The spreadsheet used to complete these risk protocols provides a means to evaluate risk, focus deliberations, and direct potential further research; not to seek rationale to avoid action.

Six general questions are used to evaluate risk:

1) What is the risk of no action?

2) What is the risk of the action to the target and the source population?

3) What is the risk of the action to species in the recipient ecosystem?

4) What is the risk of the action to higher order attributes of the recipient ecosystem?

5) What are the risks associated with potential invasion of the target to non-target ecosystems?

6) What are the ecological risks to species and services valued by society? 
These protocols are designed to support a thorough assessment of the risk of causing ecological harm due to managed relocation (e.g., low to very high), within a reasonable timeframe ${ }^{2}$. Each risk criterion is qualitatively assessed, and we do not combine risk scores into a single synthesized value. The protocols do not require or assume that new research will be conducted but are designed for resource managers to score perceptions of risk based on existing knowledge and / or expert opinion. The amount of time management dedicates to this effort is a matter of choice. If managers choose to evaluate risk using only the best available published information for a species, then evaluation can occur quickly. However, if managers choose to engage in an extensive expert elicitation to evaluate risk, as described below, the process will take longer. As humans, we are often anxious to take action to solve problems, but the loss of species as a consequence of climate change might in many cases be a slow process that takes years to come to conclusion. We therefore encourage users to distinguish urgent problems from chronic problems, while remaining aware that there is risk in waiting too long and therefore having to rush analyses, planning, and actions. We recognize these protocols may be used for urgent and immediate problems, but also we encourage resource managers to be forwardlooking and evaluate the full complement of potential management actions and their associated risks, costs, and benefits well in advance of a perceived need to act (Baron et al. 2009; Mawdsley et al. 2009; Palmer et al. 2009; Kostyack et al. 2011; Millar and Stephenson 2015).

\footnotetext{
${ }^{2}$ We do not speculate on how much time might be allotted to such a decision. We followed guidance from NPS staff that the workload projected might be reasonably completed within a month. Investment in reducing critical uncertainties (e.g., through eliciting expert opinion) may take additional time.
} 


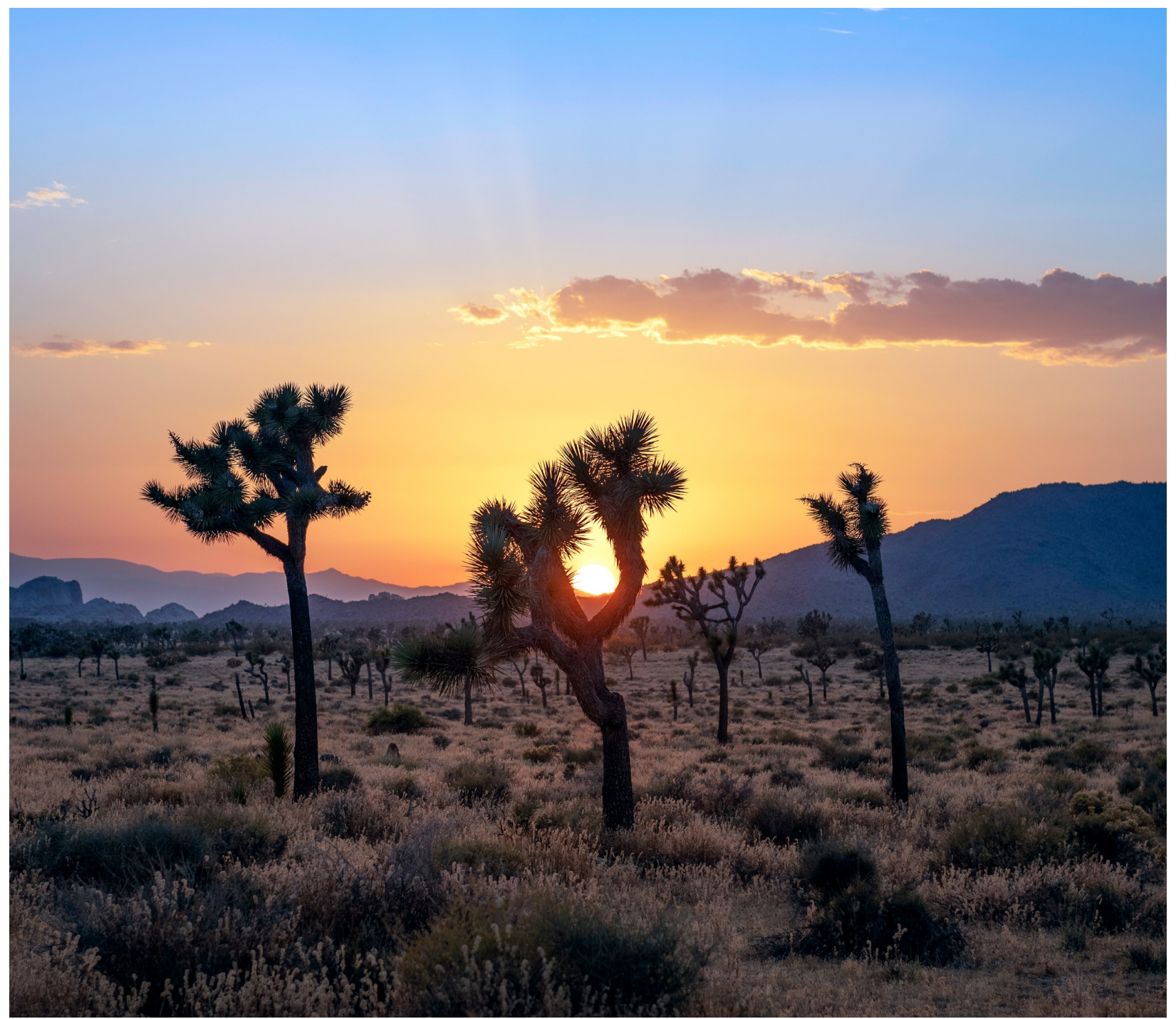

Only a few populations within the current range of the Joshua tree are projected to be sustainable under climate change, while several areas beyond its current range may become potential sites for managed relocation (Cole, et al. 2011). Credit E. Hassell, NPS 


\section{Guidance for Using the Strategy Protocols}

The risk assessment protocol is presented in a simple spreadsheet format (Figure 3). Guidance for using this spreadsheet to inform decisions on ecological risk management for managed relocation is presented in the text below.
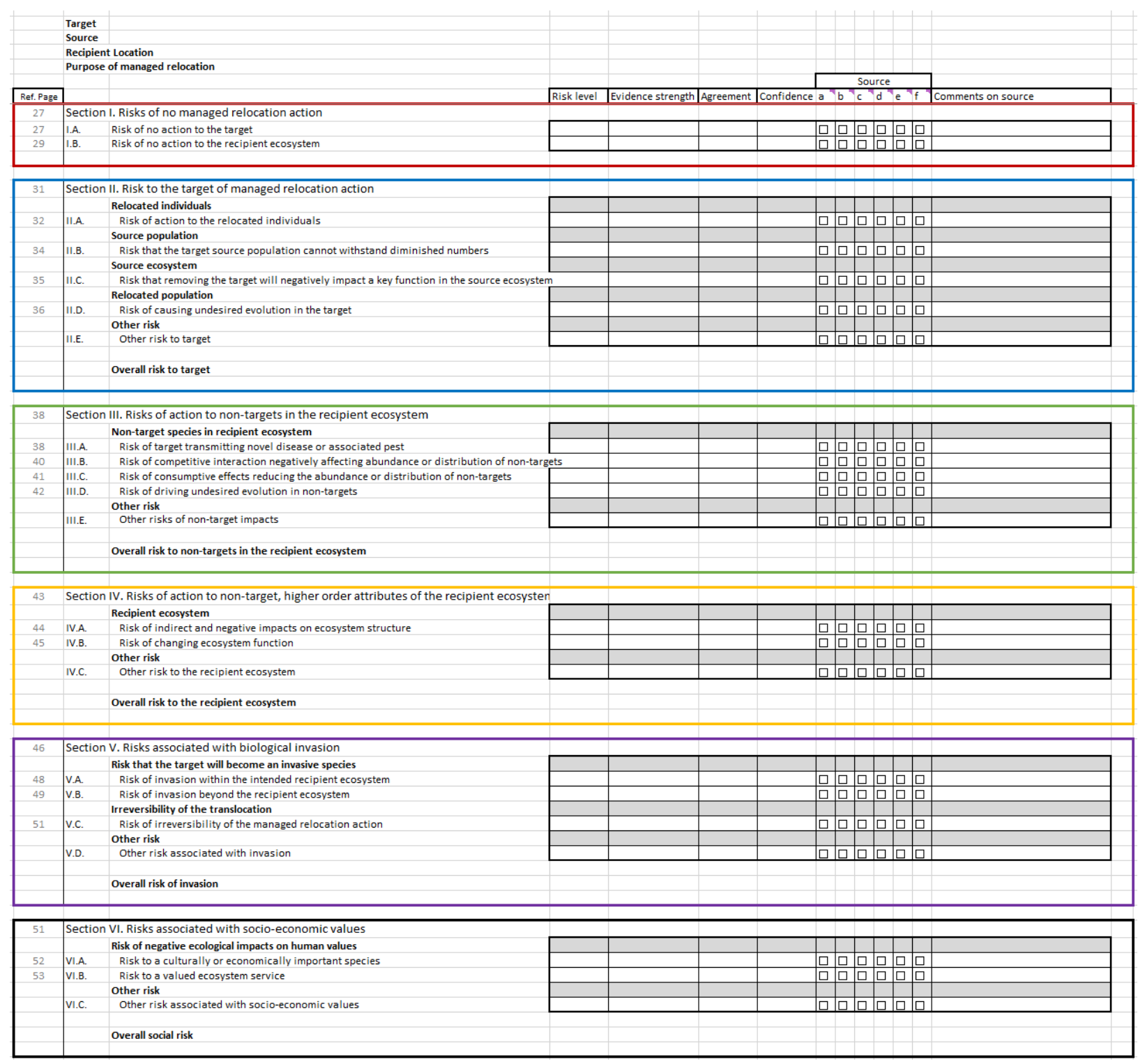

Figure 3. Screen capture of the managed relocation risk assessment tool presented in a simple spreadsheet format. Risk assessment sections presented in separate boxes. Spreadsheet rows contain section names and risk criteria. Columns for risk level, evidence strength, agreement, and confidence contain drop down lists for ranking selection. Separate columns with checkboxes provided for sources of evidence allow users to select multiple sources. Page numbers presented next to each criterion direct users to where guidance for each criterion is available in the protocols. Interactive spreadsheet tool found at https://irma.nps.gov/DataStore/Reference/Profile/2280035 (a screen reader compatible version is at https://irma.nps.gov/DataStore/Reference/Profile/2280036). 


\section{Risk Scoring, Confidence, and Uncertainty}

We adopt a process of scoring along a four-category scale using terms to describe risk (low, moderate, high, very high). For each risk criterion, we provide a suite of questions to consider when evaluating that risk. Then, we present descriptions for each category of risk. The risk scoring guidance provided for each criterion does not attempt to score all combinations of the described attributes of risk but provides examples at each level of the scale. We discourage scoring fields as "unknown" or incomplete; it is preferable to make an estimate based on professional judgement and then apply a confidence score - composed of agreement and strength of evidence - to that estimate. It is tempting to conclude that we lack sufficient information; indeed, we will always lack complete information. We strive to minimize having uncertainty preclude careful consideration and management of risk. We also include the option of selecting "N/A" for criteria that are deemed not relevant to a particular case. Further, failing to score a risk factor should not be interpreted as no risk; these are not the same.

We discourage users from simply converting qualitative risk statements into a numerical score of risk and computing an average value. This would be an inappropriate use of the risk categories for at least three reasons. First, counting and averaging introduces a false sense of precision. Second, balancing the projected benefits against the projected risks is a value judgement, not a quantitative assessment. Our task is to facilitate the decision process by allowing consideration of the full complement of potential risk. Third, average risk is not particularly informative. Many areas of risk proposed for evaluation within these protocols may have multiple sub-components (e.g., risks to recipient ecosystems generated through potential disease transmission, risks to recipient ecosystems generated through trophic interactions). It is never straightforward to decide whether the most relevant summary metric is the average risk of these sub-components or the highest individual sub-component risk. For example, when evaluating the risk of extirpation, if the risk across many attributes of life history is very low, but the risk of a species losing critical habitat is both very high and highly certain, then we would advise against averaging such scores to conclude that the risk of extirpation is moderate. In this case, the risk would be very high. Thus, considering the maximum score is likely a more appropriate way of evaluating risk scores. Potential actions that have relatively low risk scores across sub-categories are projects that should be most appealing.

Because we encourage evaluation of risk even when information is sparse, we also include fields for evaluating uncertainty. We use the Intergovernmental Panel on Climate Change (IPCC) framework for describing uncertainty and confidence in a projected outcome of an action (IPCC 2013). We focus on two dimensions of uncertainty: (1) uncertainty that comes from the type, amount, and quality of evidence as well as (2) the degree to which the available evidence is consistent, or in agreement. Little evidence and contradicting evidence can both lead to high uncertainty (Figure 4). Some risk criteria may always be difficult to assess or difficult to assess with confidence (e.g., risk of causing undesired evolution - II.D., III.D; risk of ecosystem change - IV.B). However, difficulty does not mean that it should not be assessed, and indeed, is why we also consider uncertainty. 


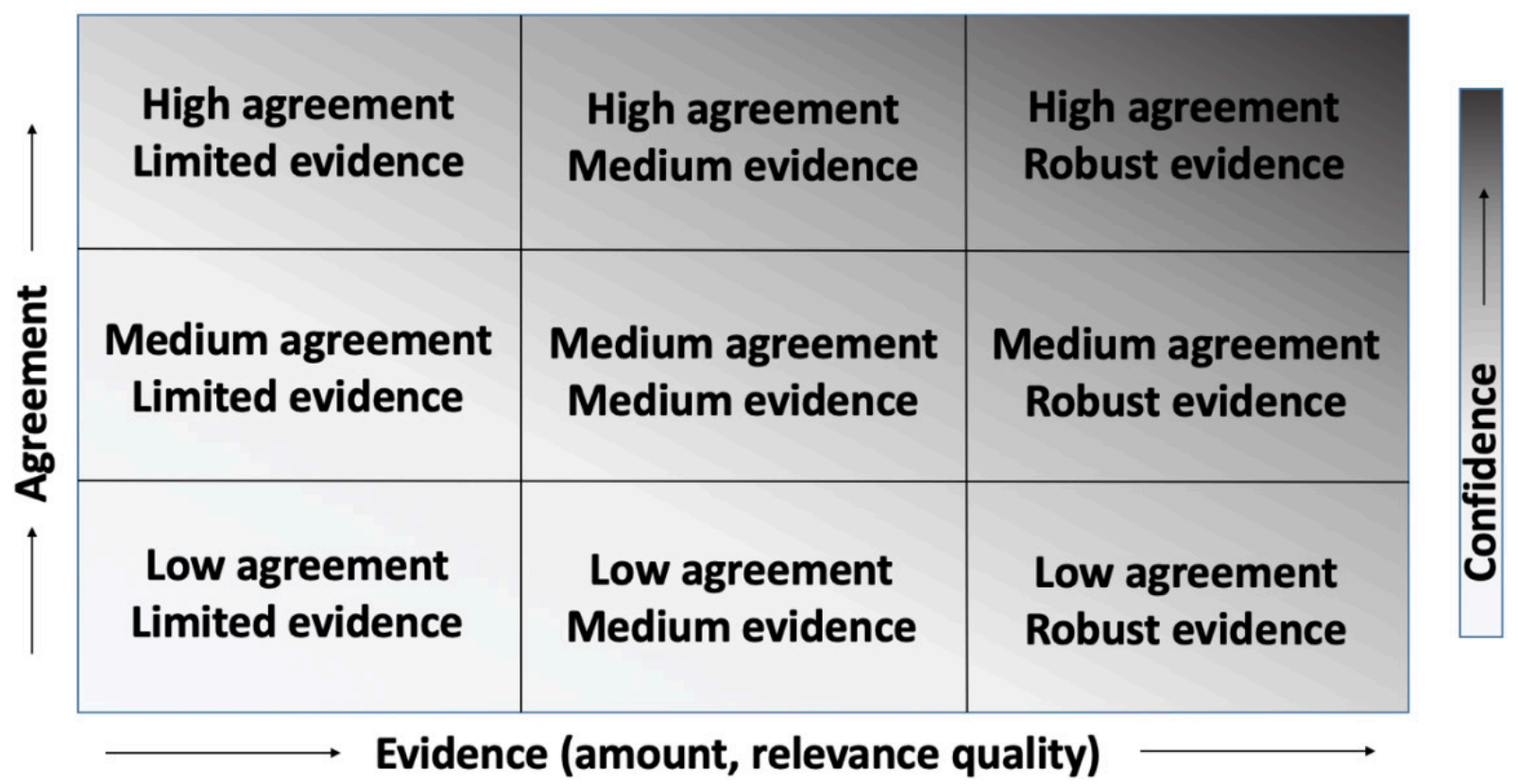

Figure 4. Confidence is a function of the uncertainty that comes from the amount, relevance, and quality of evidence, and whether the available evidence is in agreement (IPCC 2013). Confidence increases along both axes of the amount of evidence (horizontal) and the agreement of that evidence (vertical), toward the darker shading.

\section{Sources of Information}

\section{Scientific Literature and Reports}

The intent of the protocols is to provide a framework to carefully document information sources that are ultimately used to score informed assessment of risk associated with managed relocation. The peer-reviewed literature is likely to be the first major information source for users of these protocols. We understand that some attributes of risk are more available from the literature than others and some species better studied than others. Another important source of information is the non-journalarticle published literature ('grey' literature), including reports, working papers, government documents, white papers, and environmental impact assessments. Peer-reviewed and grey literature likely have purposes distinct from the managed relocation risk assessment. Thus, searching the broad body of literature is important. For example, looking at evidence of invasiveness from phylogenetically related species may aid in understanding of the invasive potential of a species (e.g., Larson and Olden 2012). However, users should scale certainty according to specificity of the literature used in the assessment. Documented analysis of the target in a similar ecosystem and geography would generate more confidence than analysis and reporting from a different ecosystem or geography, and confidence would further decrease when using a proxy taxon. 


\section{Existing assessments}

Existing tools could be employed to help with this assessment. The U.S. Fish and Wildlife Service's Ecological Risk Screening Summaries (ERSS) ${ }^{3}$ and the related Standard Operating Procedures ${ }^{4}$ apply to a wide array of species (USFWS 2018). The USDA APHIS Plant Protection and Quarantine (PPQ) weed risk assessments (WRAs) ${ }^{5}$ focus on terrestrial plants (USDA 2019). A number of state and regional Invasive/Exotic Pest Plant Councils also conduct risk analyses when developing their invasive plant lists. Finally, the IUCN publishes guidelines for classifying risk by invasive species ${ }^{6}$. These can be very useful on a more local or regional scale (e.g., https://www.calipc.org/docs/ip/inventory/pdf/Criteria.pdf). Many of these tools focus explicitly on invasive species but may offer a starting point when considering risks associated with managed relocation with a given species. At a minimum, users should check if there is an existing ERSS or WRA for that species. Any plant species should be checked against federally or relevant state noxious weed lists.

\section{Elicit expertise from species and ecosystem experts}

There are likely only a few species for which all questions in the protocol can be answered from the available literature. Using professional judgement may be a preferred process. Organizing professional judgement garnered through multiple sources can add confidence. Should a manager choose to invest resources into reducing risk uncertainty, we suggest techniques used to synthesize expert knowledge. Extending beyond the peer-reviewed or grey literature through expert elicitation is important because it can focus on the specific target and location(s), build on practice-based knowledge (Weber et al. 2014), and increase stakeholder engagement. One approach is to elicit expertise from species experts to make informed risk assessments according to their knowledge. For areas where the literature is imperfect or lacking, we recommend using a formal, but not necessarily complicated, form of expert elicitation. There are many effective methods available. We suggest using the IDEA protocol (Hemming et al. 2018). The IDEA protocol has five steps (Table 1), which we describe here briefly.

\footnotetext{
${ }^{3}$ https://www.fws.gov/fisheries/ans/species erss.html

${ }^{4}$ https://www.fws.gov/injuriouswildlife/pdf_files/ERSS-SOP-Final-Version.pdf

${ }^{5}$ https://www.aphis.usda.gov/plant_health/plant_pest_info/weeds/downloads/wra/wra-guidelines.pdf

${ }^{6}$ https://www.iucn.org/sites/dev/files/eicat_standard_version_1_may_2017.pdf
} 
Table 1. An outline of the expert elicitation process based on Hemming et al. (2018).

\begin{tabular}{|c|c|c|c|c|}
\hline $\begin{array}{l}\text { Pre-Elicitation: } \\
\text { Background }\end{array}$ & $\begin{array}{l}\text { Elicitation: } \\
\text { Investigate }\end{array}$ & $\begin{array}{l}\text { Elicitation: } \\
\text { Discuss }\end{array}$ & $\begin{array}{l}\text { Elicitation: } \\
\text { Estimate }\end{array}$ & $\begin{array}{l}\text { Post-Elicitation: } \\
\text { Aggregate }\end{array}$ \\
\hline $\begin{array}{l}\text { Background information } \\
\text { compiled. Contact and brief } \\
\text { experts on the elicitation } \\
\text { process, including how to } \\
\text { score risk and set } \\
\text { confidence intervals for each } \\
\text { risk assessment category. }\end{array}$ & $\begin{array}{l}\text { Experts individually assess } \\
\text { risk, providing rationale for } \\
\text { their judgement and their } \\
\text { uncertainty level. }\end{array}$ & $\begin{array}{l}\text { Experts are shown } \\
\text { anonymous answers from } \\
\text { each other participant along } \\
\text { with a visual summary of the } \\
\text { responses. }\end{array}$ & $\begin{array}{l}\text { Experts revise and update, } \\
\text { privately, their assessment } \\
\text { of risk and confidence. }\end{array}$ & $\begin{array}{l}\text { Estimates of risk are } \\
\text { summarized from the } \\
\text { second round of responses. } \\
\text { Experts review and discuss } \\
\text { the outcomes, add } \\
\text { commentary and address } \\
\text { any residual uncertainties. }\end{array}$ \\
\hline
\end{tabular}


Conducting the elicitation requires five roles to be represented (one person can play more than one role in a simple elicitation):

1) Problem owner: this could be the deciding manager or the person tasked with providing the assessment of risk.

2) Coordinator: Dissemination of background information and ground-rules, collection and communication of responses. Ensures the process is timely.

3) Facilitator: Manages interactions among the expert team. The Facilitator should be neutral, encourage critical thinking and full consideration of counterfactuals.

4) Analyst: Responsible for processing and analyzing responses.

5) Participants. The experts whose opinion is being elicited.

The elicitation should pose clear questions. We recommend using the questions in the protocol with your experts. In the IDEA process, the elicitation team poses these questions, or a subset, to the expert team. Experts should be credible and knowledgeable on the topic, and different sets of experts may answer different sets of questions. Strive to get at least three experts to answer each question. Here is a brief description of the process: experts are given the set of questions and individually asked to respond with both their answer and their confidence in that answer after the coordinator explains the ground rules for assessing risk and confidence. The facilitator provides all respondents with all other responses, anonymously, and asks each expert if they would like to maintain or change their assessments based on this additional information. Final answers are tallied to create a distribution of assessed risk, and a distribution of the confidence in that risk. In this way, the problem owner can determine if experts concur on risk, their confidence in that risk, or both. Depending on the decision at hand, or the stage in the decision process, managers may want to consult their Solicitor's office to ensure that input from the expert elicitation abides by the Federal Advisory Committee Act (FACA). 


\section{Risk Evaluation}

\section{Section I. Risk of no managed relocation action*}

We presume that if you are using this risk assessment protocol, there is a proposal for a managed relocation action of a target within, into, or out of, a natural area (Figure 5). Such a proposal could arise as part of an assessment of climate change vulnerability or impacts and development of potential management responses, as expressed in steps 2-4 of the Climate-Smart Cycle (Fig. 1). Alternatively, the proposed action could be a management response to threats imposed by invasive species.

* If you have already completed a thorough vulnerability assessment, you might be able to skip this section. This section is asking how vulnerable the target is to climate change without the intervention of a managed relocation action. There are numerous methods for climate change vulnerability assessments (e.g., Foden and Young 2014; Glick et al. 2011), many of which provide guidance on addressing climate change projections in terms of uncertainty and divergent projection. There are also numerous climate projections, as well as sources to find summaries of those projections (e.g., Climate Toolbox, www.climatetoolbox.org). Section I is here to emphasize the need for a complete vulnerability assessment prior to the consideration of a managed relocation action but should not be considered a substitute for a thorough assessment.

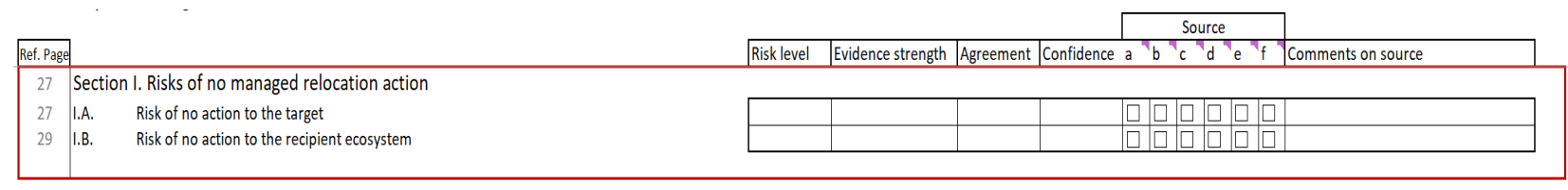

Figure 5. Screen capture of Section I of the managed relocation risk assessment spreadsheet presented in a simple spreadsheet format. Spreadsheet rows contain section names and risk topics. Columns for risk level, evidence strength, agreement, and confidence contain drop down lists for ranking selection. Separate columns with checkboxes provided for sources of evidence allow users to select multiple sources. Page numbers presented next to each criterion direct users to where guidance for each criterion is found in these protocols. Interactive spreadsheet tool found at https://irma.nps.gov/DataStore/Reference/Profile/2280035.

\section{I.A. Risk of No Action to the Target}

This subsection applies to situations where the managed relocation is motivated by the perceived inability of the target to persist in its current range(s); it is at risk of extirpation ${ }^{7}$. Here we address the risk to the target if no action is taken. This risk may be considered at multiple scales depending on relevance to the proposed actions as well as the desired objective of the action. For example, if the

\footnotetext{
${ }^{7}$ One such example would be the relocation of Joshua tree seeds or trees to cooler places upslope or northward of its current distribution in Joshua Tree National Park. See Appendix 2 for additional examples.
} 
objective of the action under consideration is conservation of a specific population, then consideration of the species globally may not be necessary.

How at risk of extirpation is the source population / target?

- Is the source population currently declining, threatened, endangered, or otherwise at risk of extirpation due, at least in part, to climate change within its current range?

- Is the predicted extirpation imminent (e.g., predicted within 10 years)?

- Do captive populations provide for the probable long-term persistence of the species in botanical gardens or zoos?

- Even if extirpation is not imminent, will earlier actions improve outcomes (e.g., ecosystem conditions, reduced extirpation risk)?

- Does the target possess sufficient adaptive capacity to allow it to tolerate current impacts/vulnerabilities to cope with or adjust to climatic changes and other threats (e.g., disease, invasive species) and persist in place (e.g., diet breadth, habitat specialization, or behavioral flexibility)? Note: in practice, this is likely to be difficult to determine.

- Are there viable strategies for long-term (e.g., 50+ years) protection of the target in situ (e.g., creating migration corridors, finding climatic micro-refugia)?

- Do barriers or other impediments prevent the target from shifting in space to allow movement into habitats with suitable climate conditions?

- Are there plausible ways to enhance the target's capacity to shift in space to track climate across the landscape on its own (e.g., establishing corridors)?

\section{$\underline{\text { Risk Scoring }}$}

We consider scoring risk of inaction on the target to be a combined function of extirpation risk, how imminent extirpation is predicted to be, and the capacity to deploy alternative management strategies.

Low: Low to moderate risk of extirpation. Extirpation is not imminent, and earlier action will not demonstrably improve the outcome of managed relocation. Moderate to high capacity to manage for persistence of the species in place.

Moderate: Moderate to high risk of extirpation, but extirpation is not imminent. Low to moderate capacity to manage for persistence of the species in place.

High: High risk of extirpation. Extirpation risk is imminent (projected to be functionally extinct ${ }^{8}$ within 10 years or currently functionally extinct in the wild), or a delay in action will reduce

\footnotetext{
${ }^{8}$ Functionally extinct is defined as a species falling to sufficiently low numbers that it no longer provides ecosystem services for which it is valued. Hence, we may be concerned with biological extinction of species, but in managing ecosystem functionality, we may be interested in functions such as predators acting as an effective control of herbivore populations or plants reducing erosion potential.
} 
genetic fitness ${ }^{9}$ of the population. Moderate capacity to support species persistence through measures other than relocation.

Very high: High risk of imminent extirpation with little or no capacity to support species persistence in situ.

Not applicable. The proposed managed relocation action is for the benefit of the recipient ecosystem and not in response to the vulnerability of the target.

\section{I.B. Risk of No Action to the Recipient Ecosystem}

This subsection applies to situations where the managed relocation is motivated by potential benefits to the recipient ecosystem under climate change ${ }^{10}$. Here we address the risk to the recipient ecosystem if no action is taken (note that a vulnerability or ecological integrity assessment of the recipient ecosystem would provide this information). An example may be a situation where, as a result of climate change, the functionality of an ecosystem is - or soon will be — significantly diminished and this loss can be attributed to lost/vulnerable species or functional groups (e.g., primary producers, major herbivores, or pollinators). A management proposal may seek to restore this functionality by moving species into the ecosystem to replace lost/declining species. A failure to take such an action may pose a risk of continued loss of functioning of the recipient ecosystem. Another example could be a case in which managers plan to restore a degraded ecosystem, and wish to do so in a way that the managed mix of species is likely to persist and maintain ecosystem functions in current or future climate conditions, rather than restoring to a past mix of species that may be unlikely to persist or maintain ecosystem functions as climate conditions change.

\footnotetext{
${ }^{9}$ Reduced genetic fitness can cover a broad range of issues and is often poorly defined. Further, many species have a demonstrable capacity to persist despite low genetic variability. What is meant here is that there is specific evidence for the target species that low genetic variability reduces persistence potential.

${ }^{10}$ One such example is the introduction of bison into a newly established grassland in the Kenai Peninsula. See Appendix 2 for additional examples.
} 


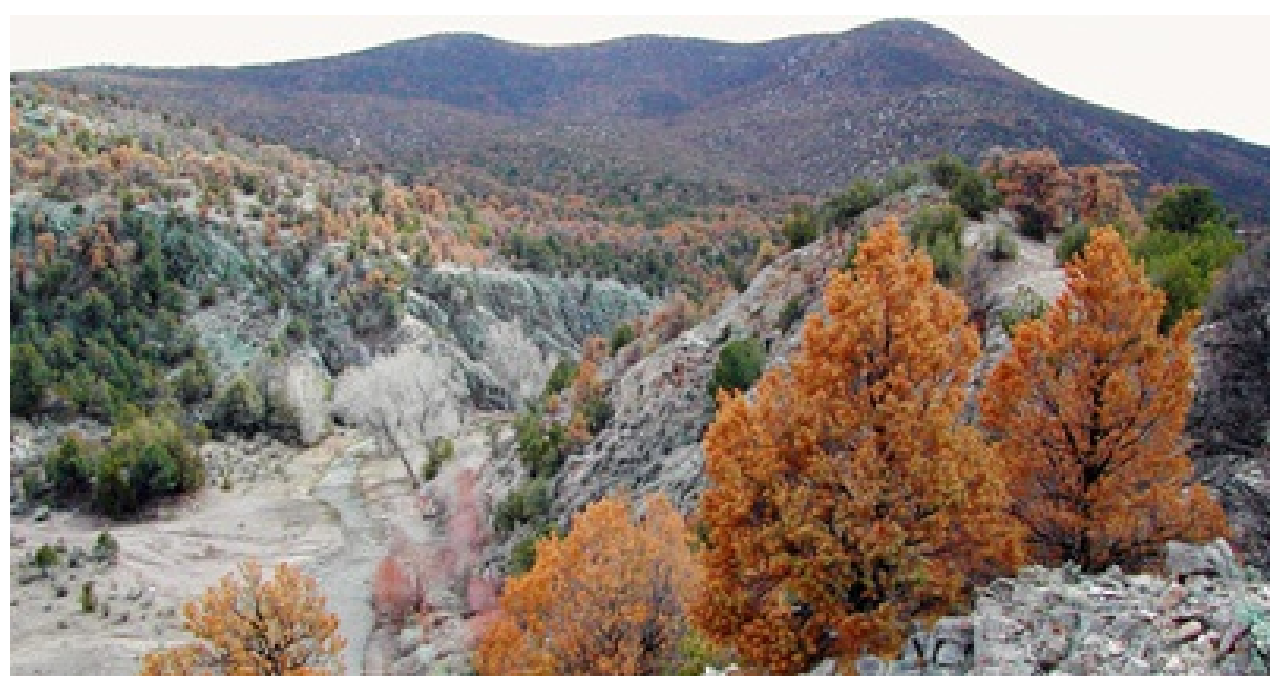

Several NPS-managed ecosystems are under climate threat. Pictured is pinyon pine (Pinus edulis) die-off in the desert southwest that has transformed the ecosystem and resulted in a decrease in avian species richness (Fair et al. 2018). Bandelier National Monument has been challenged by how to manage a drought- and fire-prone ecosystem that is losing critical foundation species as a result of climate change. Credit: US Forest Service, Southwestern Region

How vulnerable is the recipient ecosystem to climate change?

- Is the recipient ecosystem missing - or facing the loss of - a key species as a result of climate change?

- Will ecosystem function be compromised (or is it being compromised) in the recipient ecosystem due to this climate change-induced loss?

- Will the target alone resolve the degradation of ecosystem functionality in the recipient ecosystem or are additional management actions required?

- What is the degree of certainty as to whether the target species will restore the missing function?

- Are there alternative ways to add or increase the missing function in the recipient ecosystem (e.g., through surrogate species)?

\section{$\underline{\text { Risk Scoring }}$}

We consider risk of inaction on the recipient ecosystem to be a function of the risk of loss of ecosystem functionality, the capacity for the ecosystem to persist, and the availability of alternate management actions.

Low: Low to moderate risk of loss of ecosystem functionality. High capacity for the ecosystem to persist through alternate management actions. There is redundancy in the functional group.

Moderate: Moderate to high risk of loss of ecosystem functionality, but the loss is not imminent. Moderate capacity for persistence of the ecosystem through alternate management actions.

High: High, imminent risk of loss of ecosystem functionality. Low capacity for persistence of the ecosystem through alternate management actions. 
Very high: The recipient ecosystem lost a species due to climate change whose role can only be filled by the target. No alternate management action exists that would maintain the role of the extirpated species.

Not applicable. The proposed managed relocation action is for the conservation of the target. 


\section{Section II. Risk to the target of managed relocation action}

This section addresses the risks affecting relocated individuals, the proposed source population and ecosystem, and the target species as a whole (Figure 6). Whenever the persistence of the source population is a goal, it is important to evaluate risk to the source population and ecosystem. In these cases using captive populations for relocation would be preferred. In this case, risk of the proposed action to the extant population would be as low as it could be. In other cases, a relocation action may require complete removal of individuals of the target species from the source location, while retaining an interest in preserving the existing population. Assessing risk in this case is important. Finally, overwhelming risk to the extant population may result in complete removal of individuals from the extant population. In this case, the risk of harm to the extant population is high, but not a management concern. In each of these instances, it is most important to evaluate risk to the relocated individuals and the target taxon as a whole, particularly if relocated individuals are the only remaining members of the species. Generally, portions of this section may not be relevant in cases where the relocated individuals come solely from captivity. Overall, high risk in this section is a risk of action on the target and would count against a decision to take a relocation action.

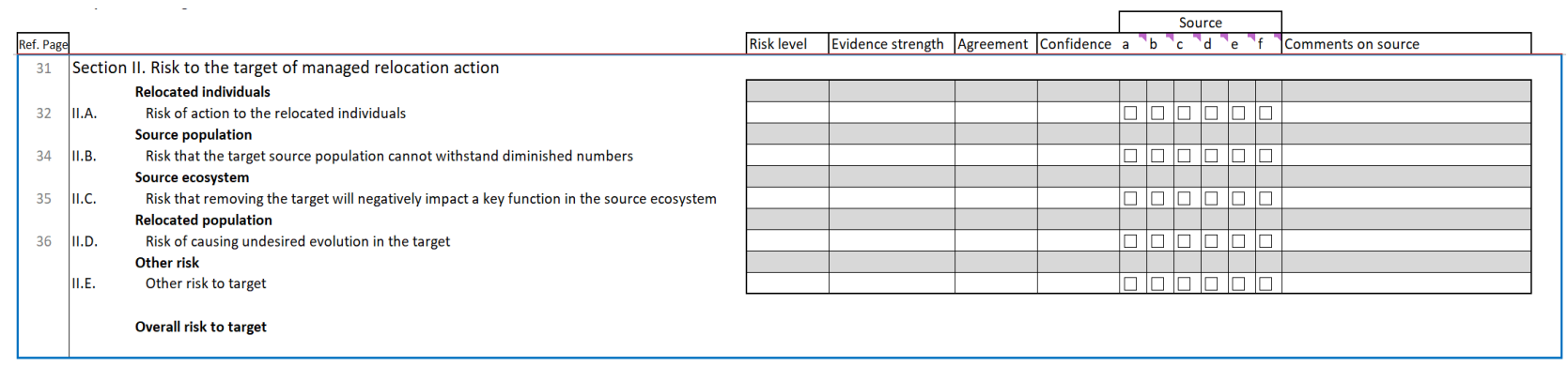

Figure 6. Screen capture of Section II of the managed relocation risk assessment spreadsheet presented in a simple spreadsheet format. Spreadsheet rows contain section names and risk criteria. Columns for risk level, evidence strength, agreement, and confidence contain drop down lists for ranking selection. Separate columns with checkboxes provided for sources of evidence allow users to select multiple sources. Page numbers presented next to each criterion direct users to where guidance for each criterion is found in these protocols. Interactive spreadsheet tool found at https://irma.nps.gov/DataStore/Reference/Profile/2280035.

\section{II.A. Risk of Action to the Relocated Individuals}

This subsection addresses the risk that the managed relocation action will have an adverse effect on the relocated individuals. Risks to the source population from which the relocated individuals are taken are addressed in subsection II.B. These individuals may come directly or indirectly (temporary captivity) from a wild population, or from captive or propagated individuals. The individuals may take the form of different relocation units. For plants, these include whole plants, seeds, remotely germinated seedlings or cuttings. For animals, the word "individual" may mean "individuals", "breeding pairs", or "breeding groups" (e.g., coteries), as appropriate to the species being relocated. 


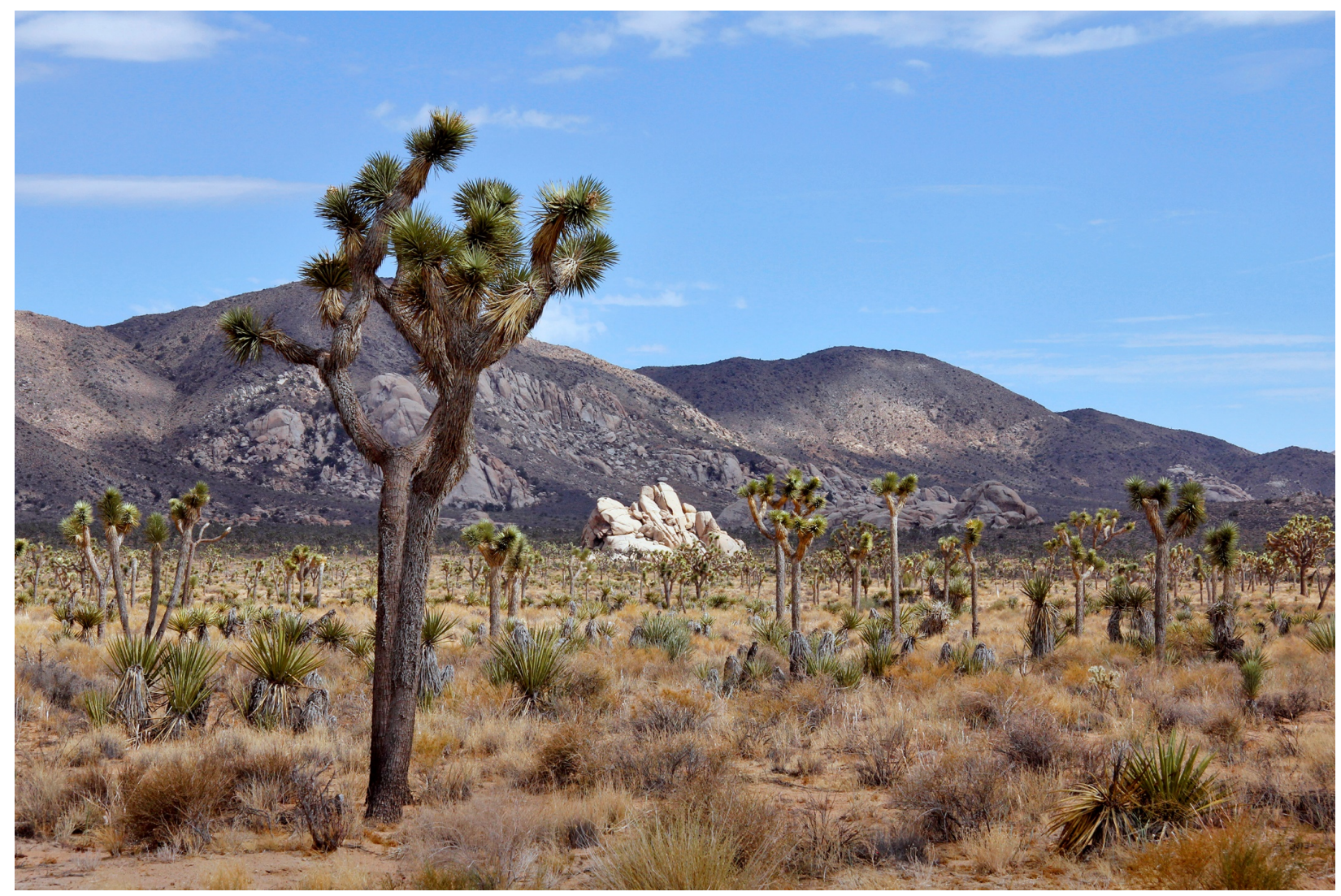

Natural areas face cases where iconic species are projected to decline or become extirpated from within the protected area (Cole et al. 2011). Assuring the long-term survival of these species may entail partnering with other organizations to assure careful propagation and husbandry in order to establish populations outside protected areas. Credit: B. Sutton, NPS

Will the relocated individuals survive and establish?

- Will individuals survive the move? (e.g., survival rate, propagule condition)

- Are individuals likely to survive and establish in the short term after relocation? (e.g., survival rate for transplanted plants, development of breeding home ranges by relocated animals)

- Consider: recipient site quality, condition and age of relocated individuals

- Is a population likely to persist in the long term? (i.e., will the relocated individuals reproduce with a net positive population growth rate, will the population tolerate extreme events in its new location)

○ Consider: number and age of relocated individuals, stochastic and Allee effects

- Are there behavioral or other life-history traits present in captive individuals that would impact their survival upon release?

- Are there pests or pathogens in the recipient ecosystem that may adversely impact the likelihood of successful relocation?

- Do we know about the life history of the target to successfully relocate it? Have similar species been relocated and were they successfully established? 


\section{$\underline{\text { Risk Scoring }}$}

We consider scoring risk of action on the relocated individuals to be a function of the condition (e.g., health, age, life stage, propagule type, behavioral traits) and number of individuals as well as their potential sensitivity to transport. Additionally, we consider whether the individuals are likely to survive in the short term, whether the recipient site is appropriately prepared to receive the target. We consider whether a population establishes or not in the long term to be a function of the condition and age of relocated individuals, number of reproductively viable individuals, and the availability of critical life history requirements (e.g., presence of key pollinator for relocated plant or nesting tree for specialist bird).

Low: Relocated individuals are mostly from the wild and are in good condition, appropriate stage or propagule for relocation, and are likely to survive the move. There are many individuals of reproductive age and the move is unlikely to negatively affect reproduction, relocated individuals are unlikely to possess any traits (e.g., germination requirements, behavioral, genetic) that will negatively affect their survival upon release. All known life history requirements are available in the recipient ecosystem.

Moderate: Moderate to low survivorship is expected. Most, but not all, known life history requirements are present. There are many relocated individuals in good condition such that immediate establishment is likely, but only some are reproductively active at the time of relocation, or likely to mature to an adult phase. Relocated individuals are from a captive population, though it is not known whether they possess traits that may adversely affect their survival in the wild ${ }^{11}$.

High: The target is sensitive to changing conditions or many individuals are in poor condition such that some, but not all, individuals will not survive during transit or release / transplanting into the new location. There is a low estimated probability of post-introduction survival. There are currently only a few individuals of reproductive age to contribute to a self-sustaining population. Some relocated individuals possess traits from their time in captivity that may negatively affect their survival in the wild. Known life history requirements missing, though possible analogs are present.

Very high: Most individuals are unlikely to survive movement or relocation. Most relocated individuals are in poor condition. There currently are no individuals of reproductive age or it would be difficult for those individuals to support a self-sustaining population (e.g., Allee effect). Relocated individuals possess traits from their time in captivity that negatively affect their survival in the wild. Some critical life history requirement is missing from recipient ecosystem.

\section{II.B. Risk That the Target Source Population Cannot Withstand Diminished Numbers}

This section is relevant in those situations where not all individuals in the source population will be relocated to the recipient ecosystem and long- or short-term persistence of the source population is of

\footnotetext{
11 "The wild" is intended to convey any location dominated by natural vegetation cover types and species are not caged or specifically managed (e.g., fed or gardened).
} 
concern. Evaluating risk within this section depends on an estimate of the number of individuals that would be relocated and the number individuals that would remain. Additionally, this section is not relevant in cases where the relocated individuals come solely from captivity or where another proposed activity (e.g., development) would destroy the source population.

Could the source population withstand the reduction in numbers associated with the relocation? Or might the relocation threaten the source population by:

- Rendering the effective population size too small to be self-sustaining?

- Creating negative genetic consequences that cannot be managed or outweigh the genetic benefits of having a geographically protected second population (e.g., inbreeding, population bottleneck)?

- Generating potential Allee effects from the reduced number of individuals?

- Creating disturbance by obtaining translocated individuals to a degree that the source population cannot withstand?

\section{Risk Scoring}

We consider the risk of action on the source population to be a function of the number of individuals remaining in the source population after relocation of some individuals, the overall viability and genetic diversity of the remaining population, and the status of the source population. Risk may also vary depending on whether the remaining population is perceived to be on the leading or trailing edge of changing climates and whether there is variation in climatic tolerance among subpopulations.

Low: The source population has been increasing or stable for the past several years and after removing individuals for relocation, there are enough remaining individuals, structure, sex and age class ratios to support long-term persistence. The source population exhibits high genetic diversity and heterozygosity.

Moderate: The source population has been stable for the past several years and after removing individuals for relocation, there are enough remaining individuals to support short- to mid-term persistence. The source population exhibits moderate levels of genetic diversity and heterozygosity.

High: The source population has been decreasing for the past several years and removal of relocated individuals might threaten short-term persistence. The source population exhibits low genetic diversity and removing individuals for relocation may result in a genetic bottleneck.

Very high: The source population has been decreasing for the past several years and removal of relocated individuals will threaten short-term persistence. The source population exhibits low genetic diversity and removing individuals for relocation will result in a genetic bottleneck and inbreeding.

Not applicable: Not applicable if relocated individuals do not originate from a wild population or if preservation of the source population is not an objective. 


\section{II.C. Risk That Removing Individuals of the Target Will Negatively Impact a Key Function in the Source Ecosystem}

While subsection II.B addresses the risk to the source population, this subsection addresses risk to the source ecosystem. We presume that if the action impacts the persistence of the source population, there may be a secondary impact on the source ecosystem. These risks are particularly relevant where taking action means removal of every individual within the source population, where persistence of the source population after relocation is unlikely, or where enough individuals are removed to impact an ecosystem function. Additionally, this section is not relevant in cases where the relocated individuals come solely from captivity.

Does the target play a key role within its extant ecosystem, and would removing individuals for relocation diminish its impact? In many cases, the true role or impact of the target may not be known a priori; therefore, this risk should be evaluated based on current understanding of the target's role in the source ecosystem.

- Does the target play a key role as an ecosystem engineer such that removal of the target's role will lead to an ecosystem level change?

- Is the target the sole prey [or forage] for another species within the source ecosystem?

- Is the target one of a group of individuals preyed upon by another species, where removal or reduction of the target may impact other prey?

- Alternatively, is the target the sole predator of another species within the source ecosystem?

- Is the target a keystone species, that is, it has disproportionate influence on food web dynamics?

- Does the target play a critical role as a mutualist or a parasite of other species in the ecosystem?

\section{$\underline{\text { Risk Scoring }}$}

We consider risk of action on the source ecosystem to be influenced, in part, by the functional role of the target in the source ecosystem and the relative impact the target has on the source ecosystem. In most cases, we evaluate this risk only in cases where relocation involves complete or near-complete removal of the target from the source ecosystem. We do this under the assumption that removing a small fraction of the existing wild population will have a minimal impact on the resident population.

Low: The target is not an ecosystem engineer. There is ample redundancy within the source ecosystem such that other species fill the role of the target species.

Moderate: There is some redundancy within the source ecosystem and there are a few other species that fill the role of the target species.

High: The target fills a critical role in the source ecosystem that may not be able to be filled by any other species.

Very high: The target is an ecosystem engineer, essential prey, or apex predator. It is unlikely that any other species will be able to fill the role of the target within the source ecosystem.

Not applicable: Not applicable if relocated individuals do not originate from a wild population. 

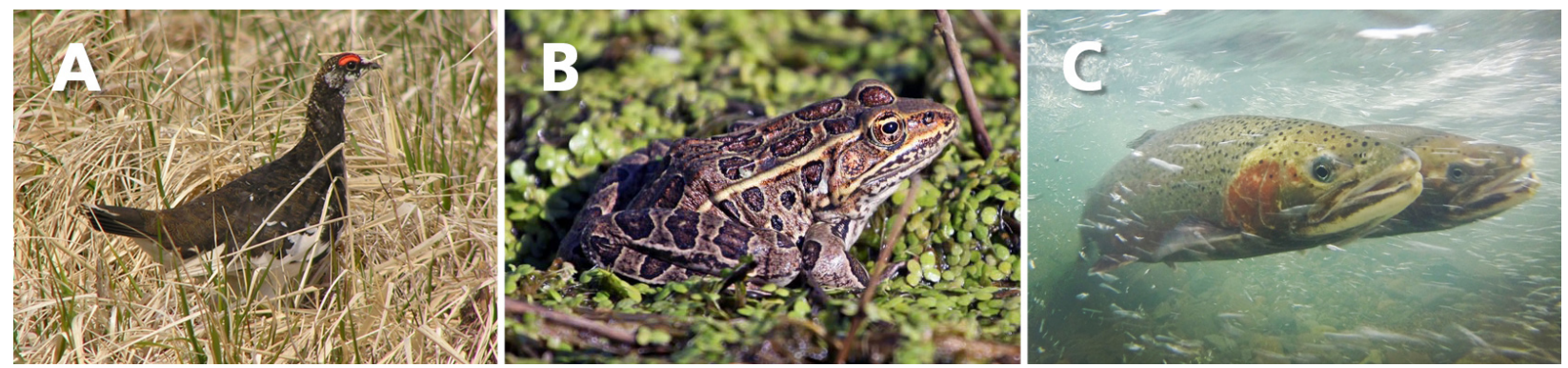

Three species for which relocation could drive undesirable evolution. A. An island restoration of Evermann's rock ptarmigan (Lagopus muta evermanni) on Agattu Island, Alaska was forced to use a very small number of founders, leaving the established populations vulnerable to inbreeding depression (Gregory et al. 2012). B. Population isolation can lead to accruing novel genotypes that do not mix well with other populations. Unique mitochondrial haplotypes identified in the northern leopard frog (Lithobates pipiens) suggest a potential for outbreeding depression with relocation (O'Donnell et al. 2017). C. Central California coast steelhead (Oncorhynchus mykiss irideus). Hatcheries and other captive breeding facilities have been noted for the propensity for increases in genetic traits that are maladaptive in the wild, leading to poorer performance of released individuals (Fraser 2008). Credit: (A) S. Ebbert, USFWS (B) G. Enslinger, USFWS (C) W. Boucher, CA Sea Grant.

\section{II.D. Risk of Causing Undesired Evolution in the Target}

This subsection focuses on the evolutionary consequences of a relocation action and assumes longterm establishment of the relocated population. This section addresses the potential for the relocated population to, over time, become genetically distinct from the source population. Additionally, the target may evolve to adapt to the recipient ecosystem or to expected future climate in a way that benefits long-term persistence of the target. In this section, we only address the risk that evolution would lead to negative outcomes (e.g., reduced fitness). Three specific mechanisms arise as having been frequently addressed in the conservation genetics literature. These are inbreeding depression as a result of the introduction of small numbers of genetically similar individuals, outbreeding depression as a result of introducing individuals with very dissimilar genotypes that do not interbreed well with local individuals, and the introduction of maladaptive traits through introducing individuals that carry traits that are detrimental in the new location. This section does not address evolution of the source population following the relocation action.

Is there a risk that creating a separate relocated population creates some unwanted evolutionary trajectory?

- Are there genetic traits (genotypes) present in captive individuals that would cause an evolutionary response (e.g., to expected future climate) that reduces the fitness of the target in the recipient site (e.g., outbreeding depression)? Is there a risk of propagating these traits?

- Is there low genetic diversity in relocated individuals, or will they comprise a small founding population that could cause unacceptable evolution in the relocated population (e.g., inbreeding depression)?

- Are the selective forces acting on the relocated population in the new location sufficiently distinct (or will become so under changing climates) that the user thinks they will exert an inordinately large selective force on the relocated population (e.g., maladaptive traits) such that 
the population may evolve away from its presumed current condition of being adapted to the presumed future climate?

- It is good to consider the generation time of the target organism relative to the pace of climate change here.

\section{$\underline{\text { Risk Scoring }}$}

Risk here is a function of the genetic diversity of the relocated individuals, the presence of undesired traits (e.g., in captive individuals), the presence in the relocation site of selective forces that are new to the relocated individuals and that are maladaptive with respect to the incoming new climate, and the number of relocated individuals.

Low: The relocated population is composed of wild individuals. Similar selective forces operate within the recipient and source ecosystems. The relocated population is large and exhibits a high degree of genetic diversity.

Moderate: The relocated population is composed of captive individuals with no known undesired traits. The relocated population exhibits moderate heterozygosity and genetic diversity, though it is not large. Alternatively, the relocated population is small, but still exhibits high genetic diversity and consists of wild individuals.

High: The relocated population is composed of captive individuals with no known undesired traits, but a low level of genetic diversity. Alternatively, the relocated population is composed of genetically diverse wild individuals, but the selective forces operating within the recipient ecosystem are different than those within the source ecosystem.

Very high: The relocated population is composed of captive or wild individuals with known undesired traits (e.g., captive individuals behaviorally maladapted to wild environments, maladaptive mutation in relocated individuals). There is a low level of genetic diversity. The selective forces operating within the recipient ecosystem are different than those within the source ecosystem. 


\section{Section III. Risks of action to non-targets in the recipient ecosystem}

This section addresses risks of managed relocation on non-target individuals and species within the recipient ecosystem and primarily focuses on direct impacts resulting from the relocation of the target (Figure 7).

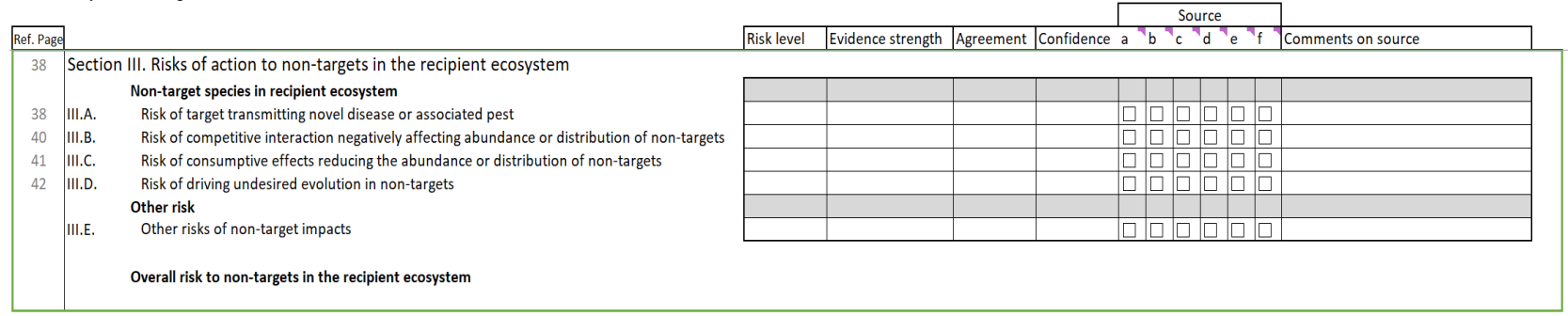

Figure 7. Screen capture of Section III of the managed relocation risk assessment protocol presented in a simple spreadsheet format. Spreadsheet rows contain section names and risk criteria. Columns for risk level, evidence strength, agreement, and confidence contain drop down lists for ranking selection. Separate columns with checkboxes provided for sources of evidence allow users to select multiple sources. Page numbers presented next to each criterion direct users to where guidance for each criterion is found in these protocols. Interactive spreadsheet tool found at https://irma.nps.gov/DataStore/Reference/Profile/2280035.

\section{III.A. Risk of Target Transmitting Novel Disease or Associated Pest}

Can the relocated species carry a disease and / or associated pest into the recipient location (e.g., strains of chytrid fungus)?

- Can disease or pest be detected and screened?

- Can disease or pest be eliminated from the relocated individuals prior to release or managed within the recipient ecosystem?

- Are relocated individuals bred, and if so, is there an available sterile technique?

\section{$\underline{\text { Risk Scoring }}$}

We consider risk of passing on a disease or pest to the recipient ecosystem to be a function of transmissibility, impacts of the disease to population health, and removal of the disease or pest. The disease can be removed either through detection and removal of carriers from the population or effective treatment of infected individuals, both of which require sensitive detection capabilities. The scale below does not attempt to score all combinations of these attributes but provides examples of where they might fall along this scale.

Low: The target is not a known carrier of a disease or pest or carries diseases or pests that are easily detected and removed prior to release in the recipient ecosystem or the disease is endemic in both the recipient and source ecosystems and generally considered low consequence to both the target and conspecific populations' health.

Moderate: The target is a known carrier of a disease or pest of which there is a mostly effective detection and removal modality available, the disease or pest is not easily transmittable to other 
individuals of its own species or another, or the disease / pest is generally considered low consequence to the target, conspecific, and non-conspecific populations' health or already present in the recipient ecosystem. Alternately, the target is a known carrier of a readily transmittable disease / pest of known negative impact to source, conspecific, and non-conspecific populations' health, but the disease / pest can be detected with a high degree of confidence and removed from the population.

High: The target is a known carrier of a disease / pest that is transmittable only to individuals of its own species of which there is minimally effective detection and removal capabilities, the disease / pest is known to have potential negative consequences to population health, and the disease / pest is not currently present in the recipient ecosystem.

Very high: The target is a known carrier of a disease or pest that is easily transmitted to individuals of its own species as well as other species and is known to have negative impacts on population health, removal is not feasible, and the disease / pest is not currently present in the recipient ecosystem.

Not applicable: The target is not a known carrier of any disease or pest of population health concern of any species.

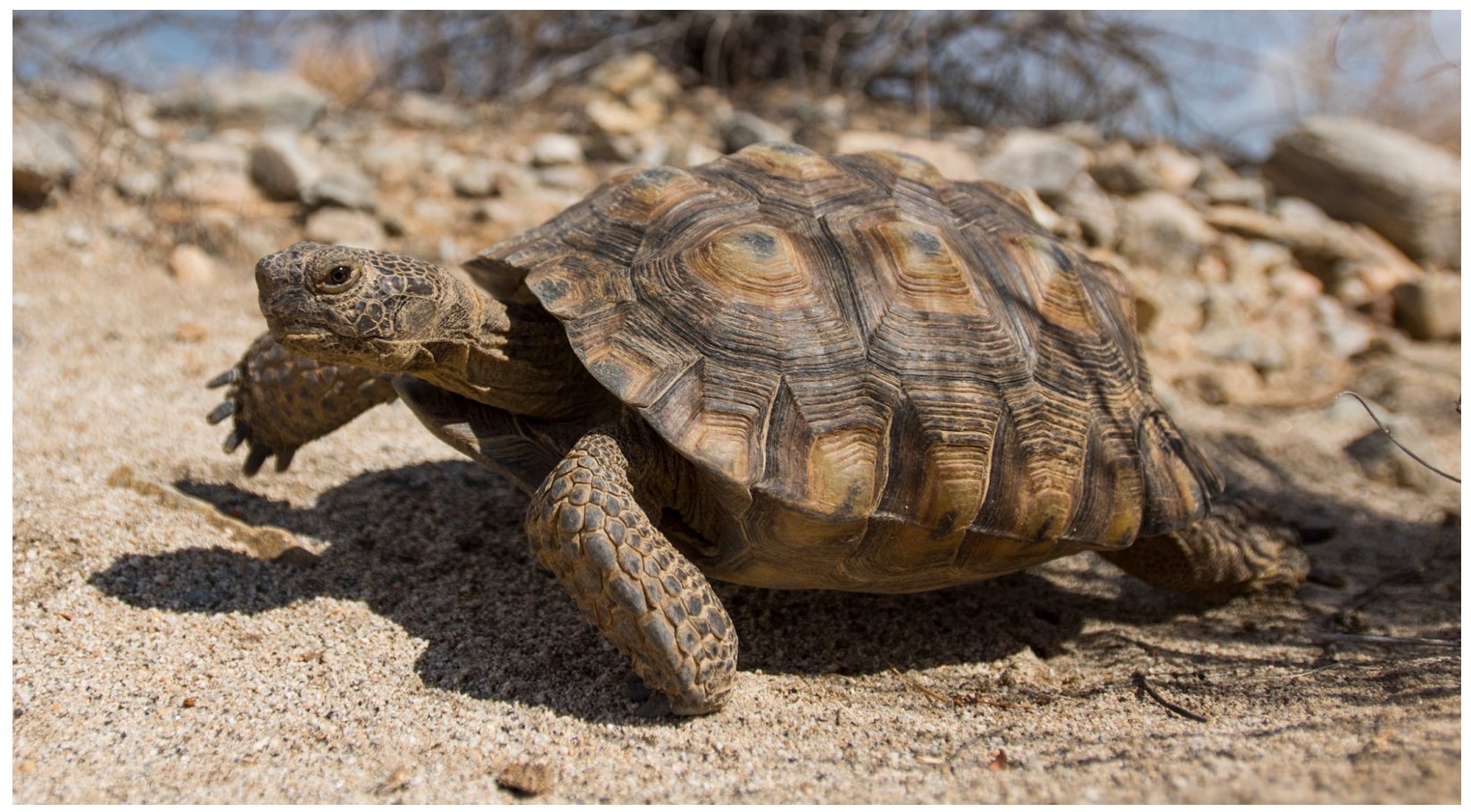

Translocation of Mojave desert tortoises (Gopherus agassizii) is considered in response to habitat loss. Inoculation of these tortoises against several microparasites that cause a detrimental upper respiratory disease can help prevent disease spread when individuals are translocated to areas with an established population (Aiello et al. 2014). Credit: B. Sutton, NPS 


\section{III.B. Risk of Competitive Interaction Negatively Affecting the Distribution or Abundance of Non-targets}

Is there risk that competitive impacts created by the target will reduce the abundance and distribution of existing species in the recipient ecosystem via competition?

- Are there closely related species in the recipient ecosystem?

- What species play similar ecological roles in the recipient ecosystem?

- Are there already multiple species performing similar roles or consuming similar resources?

\section{$\underline{\text { Risk Scoring }}$}

We consider risk of competition with other individuals in the recipient ecosystem to be a function of the degree of niche overlap of species within the recipient ecosystem, the competitive ability and conservation status of those similar species, and the availability of shared resources.

Low: There are species in the recipient ecosystem that rely on similar resources as the target. The species are currently abundant, and the availability of the shared resources is unlikely to result in adverse impacts of direct competition. The resources over which the species compete are highly abundant.

Moderate: There are species in the recipient ecosystem that rely on several similar resources as the target. The species are currently abundant and may be inferior competitors. Shared resources are moderately abundant, though somewhat limiting in the ecosystem or susceptible to external stressors [prior to the relocation].

High: There is a species in the recipient ecosystem that relies on almost identical resources as the target and this species is currently imperiled or of conservation concern. Alternatively, there are several species within the recipient ecosystem with which the target species may compete and at least one is currently imperiled. Alternatively, the target competes with another species which is abundant, but the shared resource is limited.

Very high: There are several species within the recipient ecosystem that rely on almost identical resources, are likely to be inferior competitors, and are currently threatened or of conservation concern. The resources which would be shared between the target species and other species within the recipient ecosystem are already highly limited.

\section{III.C. Risk of Consumptive Effects Reducing the Abundance or Distribution of Non-targets}

This subsection addresses the risk of managed relocation to species consumed by the target, whether the target is a predator, herbivore, omnivore, or plant (reducing seed germination, growth, and/or survival of non-target species through allelopathic effects). This includes animals (prey) and plants (forage) potentially eaten by the target species. This section only addresses direct effects of the target species on their prey or forage; other indirect food web interactions are considered separately. High predation pressure or exclusive predation may reduce the abundance of the prey or change the prey's distribution if the target expands beyond the recipient ecosystem. 
Is there a risk that consumption by the target will reduce the abundance and distribution of species in the recipient ecosystem?

- Are there any non-target species that may become locally threatened or put at risk by actions of the target?

- Are any species in the recipient ecosystem similar to species known to be consumed by the target?

- Will the target exclusively consume a single species?

- What would be the degree of consumptive pressure?

- Are any currently threatened or endangered species likely to be affected by the target?

\section{$\underline{\text { Risk Scoring }}$}

We consider consumptive risk to be a function of the number of prey options (whether the target is a generalist or specialist), the novelty of the target to species in the recipient ecosystem, as well as the status of prey and current consumptive pressure within the recipient ecosystem.

Low: The target is a generalist predator [or herbivore] and there are multiple prey [or forage] species within the recipient ecosystem that are currently abundant. Alternatively, the prey currently has few predators, and the addition of the relocated species is unlikely to reduce the prey's abundance and the prey may be able to learn how to avoid the new predator. The target is not known to be a voracious predator [or destructive grazer] and is unlikely to become one. The prey has been exposed to novel predators in the past and is capable of avoiding predation and persisting. Alternatively, the target is not known to exhibit allelopathic effects on other organisms.

Moderate: The target is a generalist predator [or herbivore], but there is only one primary prey [or forage] species within the recipient ecosystem, and it is currently abundant. Alternatively, the target is a generalist predator [or herbivore] and there are multiple prey [or forage] species within the recipient ecosystem and one is currently threatened. Alternatively, at least one prey species will not respond well to the relocated species as a novel predator and will likely decrease in abundance to an unacceptable level. Alternatively, the target may exhibit allelopathic effects on other organisms though impacts are unlikely to impact those organisms at the population level (e.g., reduced growth of individuals).

High: The target is a specialist predator [or herbivore] and the prey [or forage] species is currently abundant but with a slow rate of reproduction. Alternatively, the target is a generalist, there are multiple prey [or forage] species within the recipient ecosystem, and most are currently threatened. Alternatively, there is only one prey [or forage] species within the recipient ecosystem and it will likely be naïve to the novel predator and not be able to escape predation. Alternatively, the target is a generalist and can sometimes be a voracious predator [or destructive grazer]. Alternatively, the target may have detrimental allelopathic effects on other organisms that reduces the germination or survival of those organisms, though sympatric persistence is still possible. 
Very high: The target is a specialist predator [or herbivore], and its single prey [or forage] species within the recipient ecosystem is currently threatened. Alternatively, the prey species will likely be naïve to the novel predator and not be able to escape predation. Alternatively, the target is known to be a voracious predator [or destructive grazer]. Alternatively, the target is known to have detrimental allelopathic effects on other organisms that will reduce the growth, reproduction, and survival of other organisms.

Not applicable: The target does not consume other organisms (e.g., a plant), or is known to be a plant without allelopathic capabilities.

\section{III.D. Risk of Driving Undesired Evolution in Non-targets}

This subsection addresses the potential for the target to reduce evolutionary fitness in other species within the recipient ecosystem. Competition with the target could lead to undesirable evolution through forces such as character displacement. Predation [or consumption] by the target could lead to undesirable evolution by, for example, selective foraging on individuals within a non-target population with certain physical traits. Broadly speaking, evolutionary change is expected, especially as the recipient ecosystem and non-targets therein respond to climate change. Furthermore, evolutionary change will likely be difficult to detect and may in fact encompass positive change. So, while we expect a managed relocation action to have a potential to affect evolution, we focus here on assessing the risk of that adversely affecting outcomes.

Is there a risk that the introduction of the target will create some unwanted evolutionary trajectory in species in the recipient ecosystem?

- Is there risk that interspecific interactions (competition, predation, etc.) with the target could create an evolutionary response that reduces the fitness of a non-target species?

- Is there risk of hybridization of the target with related species (or subspecies) in the recipient location?

\section{Risk Scoring}

Here we consider risk to be a function of the likelihood that the target will create new selective forces that would act on non-target species and the potential for hybridization.

Low. The presence of the target is unlikely to create new selective forces that act on non-target species in the recipient ecosystem. There are no species within the recipient ecosystem with which the target would be capable of breeding.

Moderate. Competition with or predation by the target may favor new physical or behavioral traits in a non-target species but is likely not enough to lead to evolutionary changes in those species. There are species similar to the target within the recipient ecosystem, though it is unknown if they can interbreed.

High. Competition with or predation by the target may cause physical or behavioral traits to be fixed in a non-target species within the recipient ecosystem, though the traits are not detrimental to the growth and survival of non-targets. There is at least one species within the recipient ecosystem with which the target can breed. 
Very high. Competition with or predation by the target will likely cause physical or behavioral traits to be fixed in a non-target species within the recipient ecosystem that would be detrimental to the growth and survival of non-targets. There is at least one species within the recipient ecosystem which is likely to hybridize with the target.

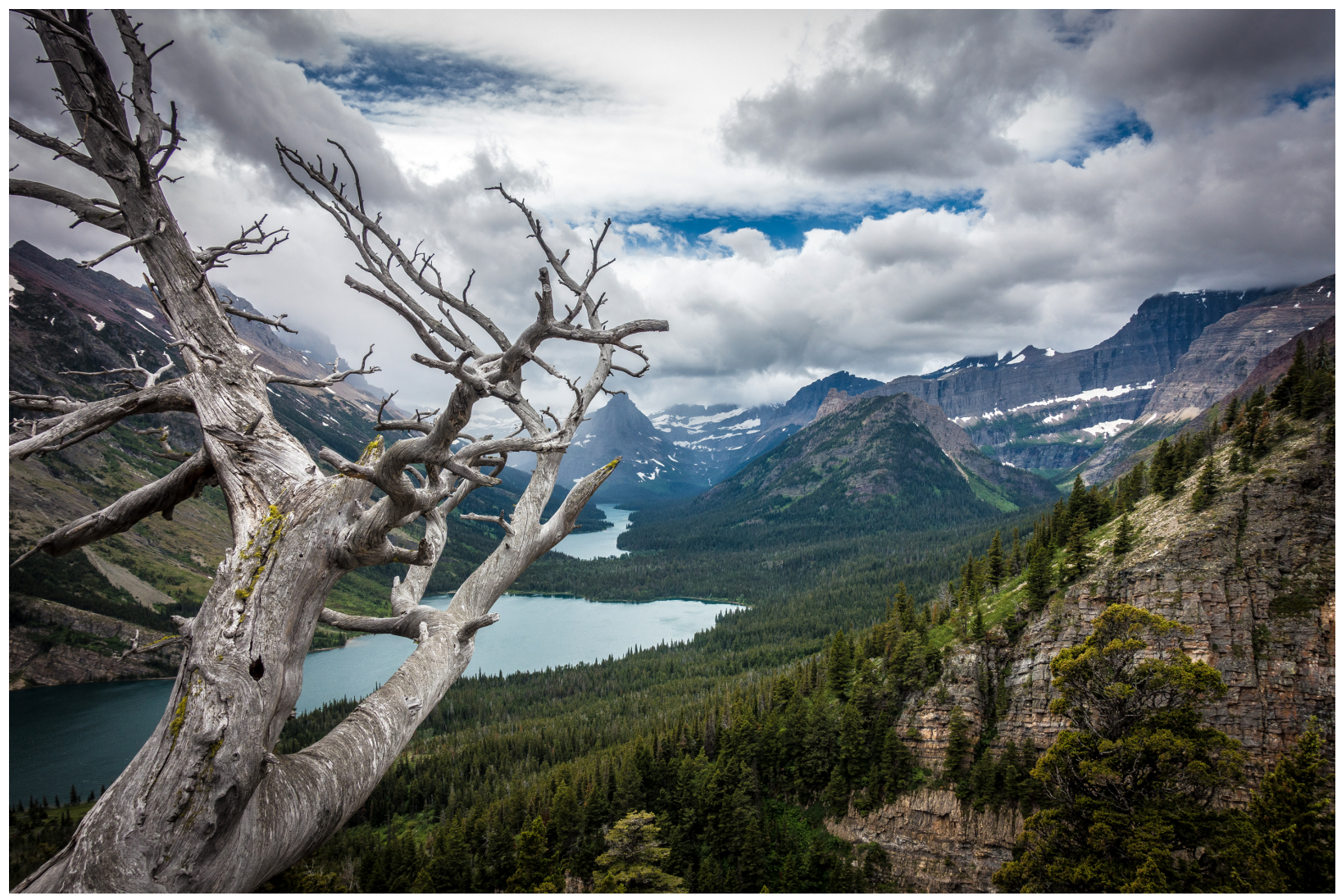

Fire suppression or intense fires, mountain pine beetles, diseases, drought, and biome shifts due to loss of area of climate suitability create a complicated picture for the future of whitebark pine. Credit: NPS 


\section{Section IV. Risks of action to non-target, higher order attributes of the recipient ecosystem}

This section focuses on higher order attributes of the recipient ecosystem, meaning community and ecosystem level responses, as opposed to the individual species responses in Section III (Figure 8). Additionally, there is a greater focus on indirect effects caused by the target, compared with the direct effects discussed in the previous section.

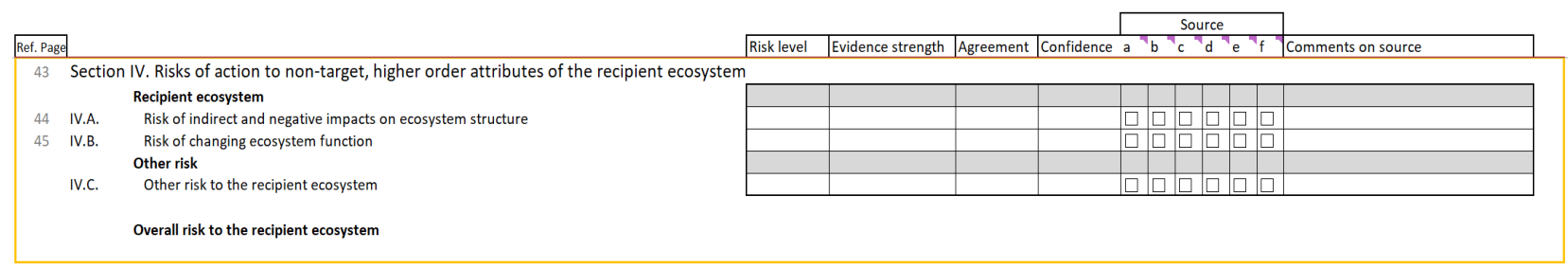

Figure 8. Screen capture of Section IV of the managed relocation risk assessment protocol presented in a simple spreadsheet format. Spreadsheet rows contain section names and risk criteria. Columns for risk level, evidence strength, agreement, and confidence contain drop down lists for ranking selection. Separate columns with checkboxes provided for sources of evidence allow users to select multiple sources. Page numbers presented next to each criterion direct users to where guidance for each criterion is found in these protocols. Interactive spreadsheet tool found at https://irma.nps.gov/DataStore/Reference/Profile/2280035.

\section{IV.A. Risk of Indirect and Negative Impacts on Ecosystem Structure}

This subsection addresses risks associated with the addition of the target to the ecosystem structure, including food webs and the relative abundance and distribution of species that comprise the community in the recipient ecosystem. Predation and competition were discussed in section III, but only with respect to species directly consumed by or in competition with the target. This risk criterion addresses indirect effects that may be mediated by a species preyed upon by the target or other effects that may cascade through the ecosystem.

How will the relocated target indirectly affect species in and structure of the recipient ecosystem?

- Does the target negatively and indirectly affect the recipient ecosystem (e.g., increase competition among consumed species, alter food web dynamics)?

$\circ$ What species may become abundant because their competitor is being preyed upon by the target, such that there is a change in the dynamics of competition between those prey species?

$\circ$ What species may change abundance because their competitor is preying on the target, such that there is a change in the dynamics of competition between those prey species?

- Is there potential for higher-level ecosystem impacts, such as a trophic cascade?

- For targets that are animals, can a disruption to the ecosystem indirectly affect plant species' abundance or distribution?

- For targets that are plants, can a disruption to the ecosystem indirectly affect animal species' abundance or distribution?

- Are there other indirect species interactions? 


\section{$\underline{\text { Risk Scoring }}$}

We consider risk to the recipient ecosystem structure to be a function of whether the target is a generalist or specialist consumer, the ability of all species at the same trophic level to exploit or resist the target equally, the status of species within the recipient ecosystem that would indirectly interact with the target, and complexity of the food web or its susceptibility to a trophic cascade.

Low. The target is a generalist predator [or grazer]. Species at the trophic level above the target will be able to equally exploit the target and all are abundant. The ecosystem / food web is complex with ample redundancy.

Moderate. The target is a generalist predator [or grazer] but might forage more strongly on some species over others. Species at the trophic level above the target will be able to equally exploit the target, but one is currently threatened or endangered. The ecosystem / food web is overall complex, but there is little redundancy surrounding the trophic level of the target.

High. The target is a specialist predator [or grazer]. Only one resident species would be able to prey upon the target possibly leading to an increase in that resident species' abundance, but the resident species' competitors are currently abundant. The ecosystem / food web is simple, and the addition of the target could lead to a trophic cascade.

Very high. The target is a specialist predator [or grazer] and could allow competitors of the target's prey [or forage] to become destructively over-abundant. Only one resident species would be able to prey upon the target possibly leading to an increase in that resident species' abundance, and resident species' competitors are threatened or endangered. The ecosystem / food web is very simple and has experienced trophic cascades in the past.

\section{IV.B. Risk of Changing Ecosystem Function}

While III.B.1 addresses indirect and higher order effects on other species in the recipient environment, this subsection addresses higher order effects on the recipient ecosystem. This includes the role the target would play in the recipient ecosystem, or through changes managers would have to make to the recipient ecosystem in order to accommodate the target.

How will the target affect ecosystem function of the recipient ecosystem?

- Does the target have capacities as an ecosystem engineer that may have ecosystem-level impacts?

- Does the target provide or alter any other ecosystem functions that might impact the recipient ecosystem, such as net primary productivity, carbon storage, water flows or nutrient cycling?

- Does the relocation action require management changes in the recipient ecosystem to accommodate the target?

\section{$\underline{\text { Risk Scoring }}$}

We consider risk of changing ecosystem functions to be a function of the role of the target within the ecosystem and management actions required to accommodate the target. These possible risk criteria are not exhaustive in terms of the higher order effects the target could have within the recipient ecosystem but are examples of how the target could impact the function of the recipient ecosystem. 
Low. The target is not an ecosystem engineer in the source ecosystem and does not provide a function that does not already exist in the recipient ecosystem or replaces / restores previously lost function. Managers will not be required to alter the recipient ecosystem in order to accommodate the target.

Moderate. The target provides functions in its source ecosystem but only in conjunction with another species not present in the recipient ecosystem. Managers will need to initially alter the recipient ecosystem (e.g., build temporary structures).

High. Managers will need to temporarily remove from the recipient ecosystem local populations of one or more species or introduce ${ }^{12}$ a species in a limited manner in order to accommodate the target (e.g., site preparation entailing clearing the recipient site of vegetation or introducing a host species for the target species to consume).

Very high. The target can substantially alter an ecosystem function. Managers will need to permanently introduce ${ }^{12}$ a species into the recipient ecosystem or remove the local population of one or more native species from the recipient ecosystem in order to accommodate the target (e.g., introduction of a host plant required by the target or removal of a local population of a species likely to consume the target).

\footnotetext{
${ }^{12}$ Introduction of any accompanying taxon, especially if the recipient ecosystem was not within the historical range of the accompanying taxon, would require second risk assessment focused on the accompanying taxon.
} 


\section{Section V. Risks associated with biological invasion}

This section addresses the risk that the relocated individuals will establish and cause changes in the recipient ecosystem to the degree that they could be considered undesirable (Jeschke et al. 2014). Many of the risks identified in previous sections constitute such undesirable consequences. Here we consider whether such undesirable consequences may be exacerbated by expansion of the target in the recipient ecosystem and beyond its planned expansion range (e.g., some set of ecosystems within some geographical boundary). In some cases, resulting ecosystem changes are dramatic and may result in the extinction of extant native species or radical changes in ecosystem functioning. This section considers the risk that the target will expand its population beyond its planned expansion range to become an undesirable non-native species. This section only examines population expansion in the context of a risky outcome, or if/when this expansion becomes a negative outcome. Additionally, this section evaluates the irreversibility of the relocation action and the risk that the relocated population cannot be controlled if limiting population expansion beyond the planned expansion range becomes necessary. The feasibility and probability of a successful managed relocation action, where the target establishes and spreads as intended without negative ecological impacts, is not evaluated here. We note that there are significant nuances in definitions related to non-native and invasive species (Lockwood et al. 2013). There is also likely to be uncertainty in considering a species 'non-native' if moved only a short distance. Our intent here is not to evaluate the risk of becoming defined by a federal policy as invasive so much as to evaluate the risk of causing harm as a consequence of unintended increase and expansion of the target.

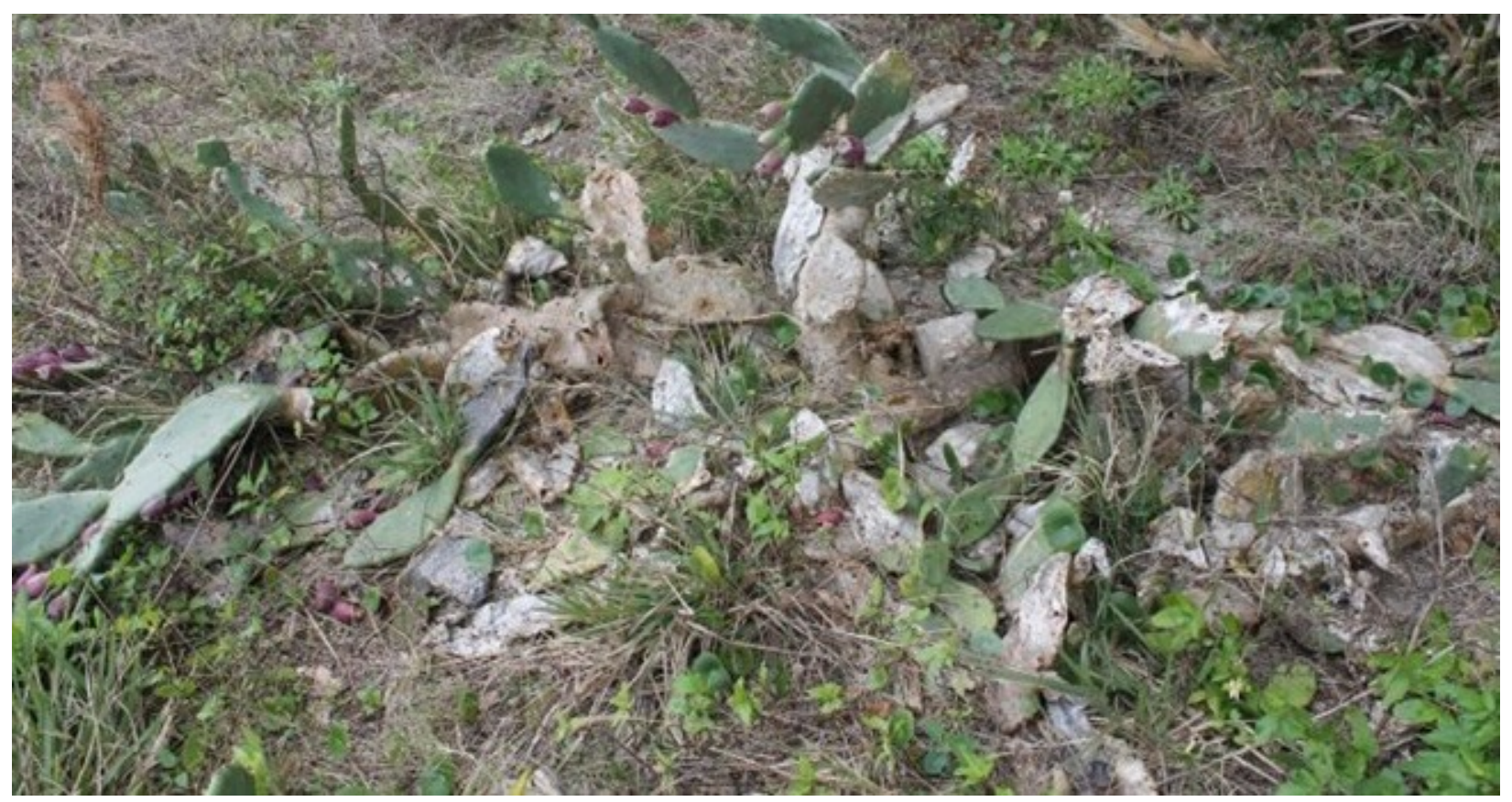

Though relocated for other reasons, the spread of a cactus moth (Cactoblastis cacotrum) illustrates how a well-meaning introduction can lead to harmful invasion. The cactus moth was released in the Caribbean as a biocontrol agent, but has skipped islands and colonized south Florida where it causes necrotic tissue damage and threatens native Opuntia species (Zimmerman et al. 2001). Credit: NPS 
Several existing resources will specifically help when considering the risk of invasion (Figure 9). These include the U.S. Fish and Wildlife Service's Ecological Risk Screening Summaries (ERSS) and the related Standard Operating Procedures (USFWS 2018), which apply to a wide array of species. The USDA APHIS Plant Protection and Quarantine (PPQ) weed risk assessments (WRAs) focus on terrestrial plants (USDA 2019). Several state and regional Invasive/Exotic Pest Plant Councils also conduct risk analyses when developing their invasive plant lists. Finally, the IUCN publishes guidelines for classifying risk by invasive species (IUCN 2017b).

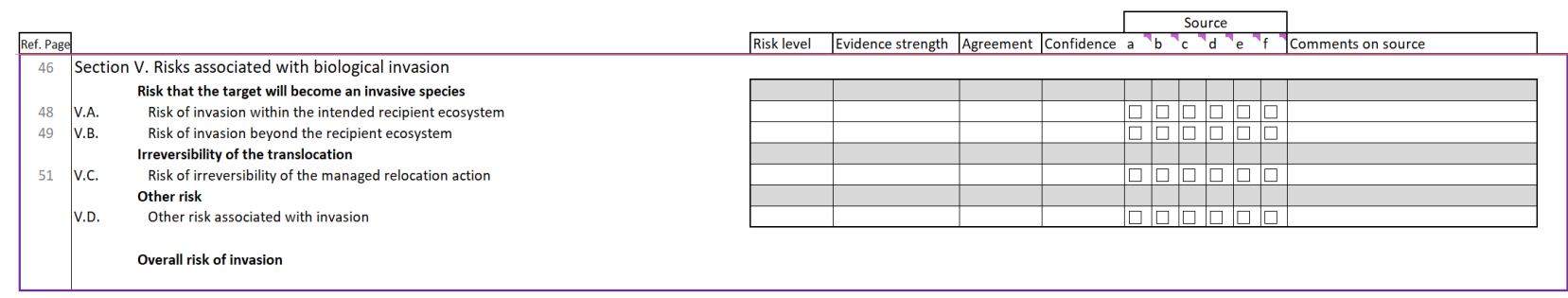

Figure 9. Screen capture of Section $\mathrm{V}$ of the managed relocation risk assessment protocol presented in a simple spreadsheet format. Spreadsheet rows contain section names and risk criteria. Columns for risk level, evidence strength, agreement, and confidence contain drop down lists for ranking selection. Separate columns with checkboxes provided for sources of evidence allow users to select multiple sources. Page numbers presented next to each criterion direct users to where guidance for each criterion is found in these protocols. Interactive spreadsheet tool found at https://irma.nps.gov/DataStore/Reference/Profile/2280035.

\section{V.A. Risk of Invasion Within the Intended Recipient Ecosystem}

This subsection addresses the risk of the target becoming an invasive species within the recipient ecosystem. We approach this section with the understanding that establishment at some level is the goal of the relocation action, but not if it is to the detriment of the recipient ecosystem or species therein. Here we consider features of the target that could allow it to become intolerably invasive.

Is the target species highly invasive?

- Does the target exhibit properties commonly found in invasive species (high reproductive rates, dominant control over resources, aggressive, etc.)?

Risk of invasion within the recipient ecosystem is a function of biological and ecological characteristics associated with invasive species (Table 2). Invasive species may exhibit a few to most of these characteristics and there are exceptions. However, this list provides some guidance towards understanding what characteristics may increase a species' invasiveness. Additionally, not all characteristics listed here will apply to every taxon. Furthermore, some of these characteristics may actually be beneficial for the relocation of the target, if they improve the likelihood of establishment following relocation. Therefore, the risk is more so that the target exhibits an invasive capability that is greater than the recipient ecosystem can tolerate. 
Table 2. Representative characteristics of many invasive species (Baker 1965; Roy 1990; Sakai et al. 2001; Marchetti et al. 2004; Hayes and Barry 2008) and how those traits may be related to invasion (modified from Olden et al. 2011).

\begin{tabular}{llll}
\hline Characteristic Type & Characteristics & $\begin{array}{l}\text { Traits linked to increased } \\
\text { likelihood of Invasion }\end{array}$ & $\begin{array}{l}\text { Traits linked to decreased } \\
\text { likelihood of Invasion }\end{array}$ \\
\hline Geographic / abiotic & Native distribution & Broad, extensive & Narrow \\
Geographic / abiotic & Abundance within distribution & High & Low \\
Geographic / abiotic & $\begin{array}{l}\text { Environmental tolerance / niche } \\
\text { breadth }\end{array}$ & Wide & Narrow \\
Geographic / abiotic & $\begin{array}{l}\text { Tolerance of disturbance or human } \\
\text { presence }\end{array}$ & High & Low \\
Ecological & Competitive ability / aggressiveness & Good, high & Poor, low \\
Ecological & $\begin{array}{l}\text { Trophic specialization (e.g., diet } \\
\text { breadth) }\end{array}$ & Broad & Low \\
Ecological & $\begin{array}{l}\text { Ecological specialization (e.g., } \\
\text { breeding system) }\end{array}$ & Generalist & Narrow, specialist \\
Demography / life history / genetics & Genetic variation & High & Low \\
Demography / life history / genetics & Fecundity & High & Low \\
Demography / life history / genetics & Offspring survival & High & Low \\
Demography / life history / genetics & Potential population growth rate & High & Low \\
Demography / life history / genetics & Mobility / dispersal capacity & High & Low \\
Other information & History of invasion in other locales \\
Other information & History of closely related taxa being & Yes & Yes \\
\hline
\end{tabular}




\section{$\underline{\text { Risk Scoring }}$}

Risk scoring in this section is particularly challenging. The ecological literature is replete with examples of invasive species carrying few characteristic traits of invasive species; as well as species carrying numerous traits, yet not being invasive. We can provide little guidance other than to examine the attributes of the target, especially the history of invasiveness of this taxon and closely related ones to make the best overall judgement of invasion potential.

Low: The target does not exhibit the characteristics of invasive species described in Table 2. The target has not been invasive elsewhere, and closely related species have not become invasive when transplanted or moved.

Moderate: The target exhibits few characteristics of invasive species (Table 2), or there is evidence of the target, or close relatives, not behaving in an invasive manner when provided the opportunity through introduction into new locations.

High: The target exhibits many of the characteristics of invasive species (Table 2). There is some evidence of the target or related taxa becoming invasive

Very high: The target exhibits most of the characteristics of invasive species (Table 2). There is a known history of the target, or closely related taxa, becoming invasive when moved to new locations.

\section{V.B. Risk of Invasion Beyond Recipient Ecosystem}

This subsection views unplanned and undesirable spread beyond the recipient ecosystem and associated undesirable impacts as a negative consequence of managed relocation. We approach this section with the understanding that in some cases, spread beyond the introduction site within the recipient ecosystem may frequently be the objective. We expect that planning will include consideration of the benefits of spread beyond the introduction site within the recipient ecosystem. However, examples of undesirable spread to a new and unintended ecosystem are also plausible, for example if a species of snail is introduced to coastal estuaries, but then unexpectedly invades nearby freshwater wetlands and river systems. Here we consider the risk of unwanted or undesirable spread and/or impact.

Risks of invasion beyond the recipient ecosystem. How likely is it that relocated individuals will spread beyond the introduction ecosystem(s)?

- What is the predicted or desired extent of spread?

- If limited establishment is desired, is the species likely to spread beyond that and will the spread cause undesirable impacts in the extended area?

- Will human behavior enhance or exacerbate problems with species spread?

- Do the risks identified for the recipient ecosystem impact surrounding areas to an equal, greater, or lesser extent (i.e., are non-target impacts discussed in section III different in neighboring ecosystems vs. in the recipient ecosystem)?

- Does the target have a high dispersal rate such that extended spread is likely? 
- Do surrounding ecosystems face other threats that make them more vulnerable to invasion by the target?

\section{$\underline{\text { Risk Scoring }}$}

We consider risk of invasion beyond the recipient ecosystem to be a function of the dispersal ability and environmental requirements of the target, the vulnerability of surrounding ecosystems, the desired extent of establishment, as well as the characteristics described above in V.A.

Low. The target has very low dispersal ability. The target can only survive under specific conditions not found in surrounding ecosystems. Surrounding ecosystems experience few threats. There is no limit to the desired extent of establishment.

Moderate. The target has moderate dispersal ability but is capable of long-distance dispersal. The target has some flexibility and can survive under different circumstances but only thrives under specific conditions not found in surrounding ecosystems. Surrounding ecosystems are currently healthy, though subject to other threats.

High. The target has high dispersal ability, though it often disperses short distances. The target can survive well in surrounding ecosystems. Surrounding ecosystems are moderately vulnerable and currently experience other threats.

Very high. The target has high dispersal ability and often disperses far distances. The target exhibits broad environmental tolerance and thus is likely to thrive under different conditions found within and around the recipient ecosystem. The desired extent of establishment is very narrow and restricted to the recipient ecosystem, and the target is likely to spread beyond the desired extent. The surrounding ecosystems are vulnerable and currently face other threats.

\section{V.C. Risk of Irreversibility of The Managed Relocation Action}

This subsection addresses the risk the target cannot be controlled should it become an invasive species. This section builds from the preceding sections which address features that would make the target invasive. Here we consider the ability to control the target or reverse the managed relocation action should negative consequences occur. Additionally, we consider only the biological features of the target relevant to population control.

If population expansion exceeds expectations or unintended consequences occur, is managing overabundance and/or distribution possible or feasible?

- How trackable / detectable is the relocated species?

- How feasible is population removal?

- Does the species create a persistent seed bank?

\section{$\underline{\text { Risk Scoring }}$}

We consider the risk of irreversibility to be a function of the detectability, mobility, and reproductive rate of the target. 
Low. The target is easy to detect and has limited mobility. The target reproduces slowly and exhibits delayed time to reach sexual maturity. The target is easily removed.

Moderate. The target is easy to detect and has moderate mobility but exhibits low propensity to disperse large distances. The target has a moderate reproductive rate.

High. The target can be difficult to detect, but often remains around the same area. The target has a high reproductive rate but reaches sexual maturity later in life.

Very high. The target is difficult to track or detect and is highly mobile. The target has a high reproductive rate and reaches sexual maturity relatively early in life. The target is resistant to removal treatment.

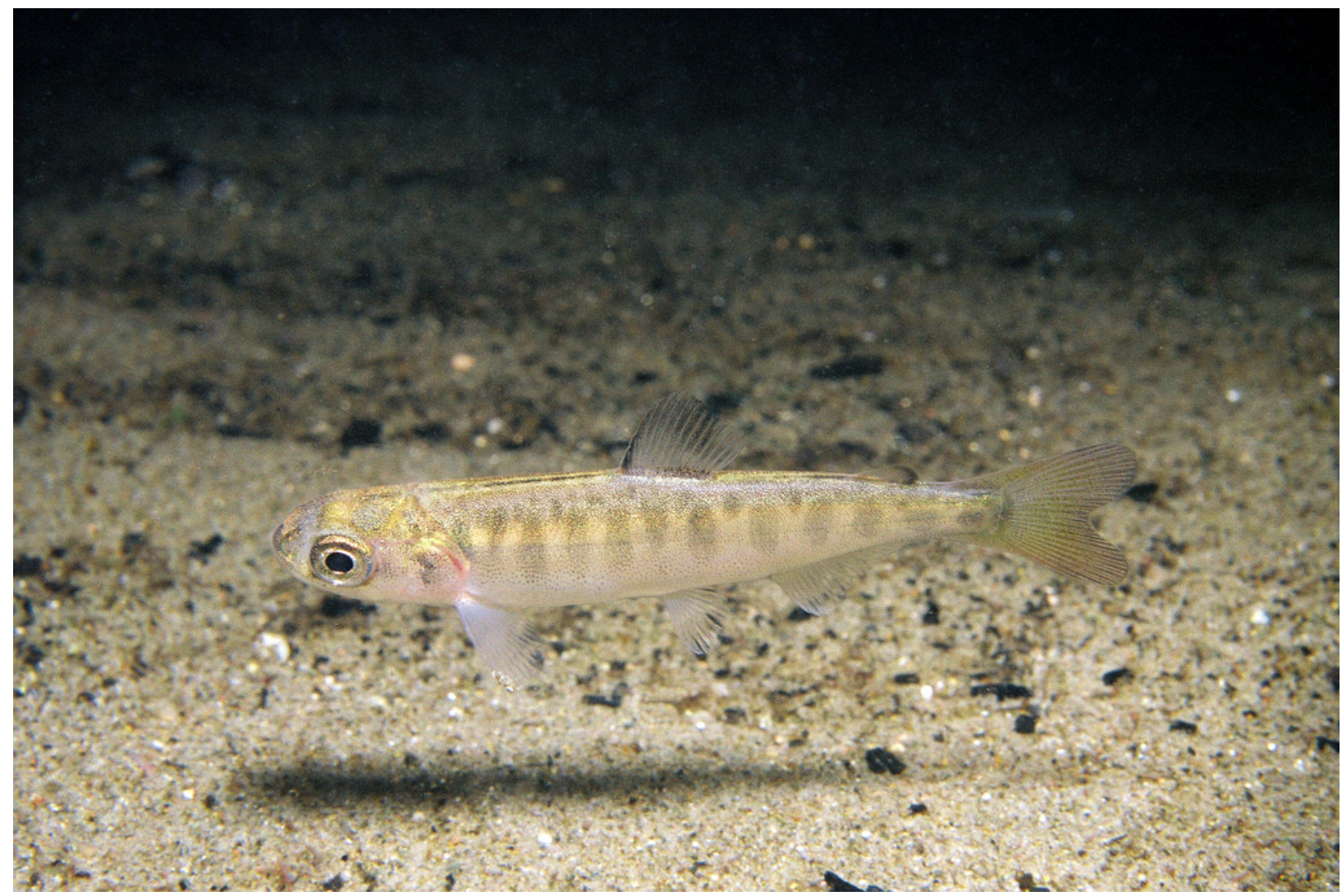

Juvenile chinook salmon. Physiological and phenological responses at various life stages complicate our ability to project long-term adaptive capacity of some species to climate change, such as salmonids. Credit: Roger Tabor, USFWS 


\section{Section VI. Risks associated with socio-economic values}

It is important for decision makers to consider the many risks of managed relocation beyond ecological risk. This section addresses some social and economic risks as they relate to ecological impacts associated with the managed relocation action. This section is not meant to be a comprehensive assessment of the ethical, social, and economic costs of moving a species. Rather, it introduces some of these issues that should be considered before an action is taken. Additionally, some impacts described in this section are closely related to how species are classified as invasive based on the federal definition (see Appendix A), which focuses on the likelihood of impacting human values and concerns; section $\mathrm{V}$ focuses more on biological impact.

For social risks, we restrict our focus to the potential for an action to impose an ecological risk on socially valued resources, such as a managed relocation that impacts the capacity to interact with wildlife. However, managed relocations will likely have other social impacts not covered here. For example, managed relocation actions may prompt both vocal public support and resistance (e.g., relocation of top predators). Alternatively, the action may require the redistribution of resources away from or preclude a more publicly favorable action. A managed relocation may also impact other human actions. For example, relocation of an endangered fish may first require the removal of a non-native but valued recreational fish species. Moving an endangered species may also impose constraints or responsibility on members of the public, especially if the species spreads beyond the recipient ecosystem onto neighboring lands, privately-owned or used by members of the public. Additionally, there may be instances where socio-economic values may lead to ecological risk (e.g., political hostility towards a management action can translate into direct actions that threaten the relocated taxon, recipient ecosystem, or managers themselves). All of these social risks are important to consider within the broader evaluation of managed relocation and can be evaluated and incorporated into formal decision making processes (Bennett et al. 2017; Robinson et al. 2019). However, these risks are directly associated with human values rather than ecological risks, which is the focus of these protocols.

This section addresses the ecological consequences of managed relocation that have implications for social and economic values. These criteria build from concerns discussed in sections III and IV (Figure 10). 


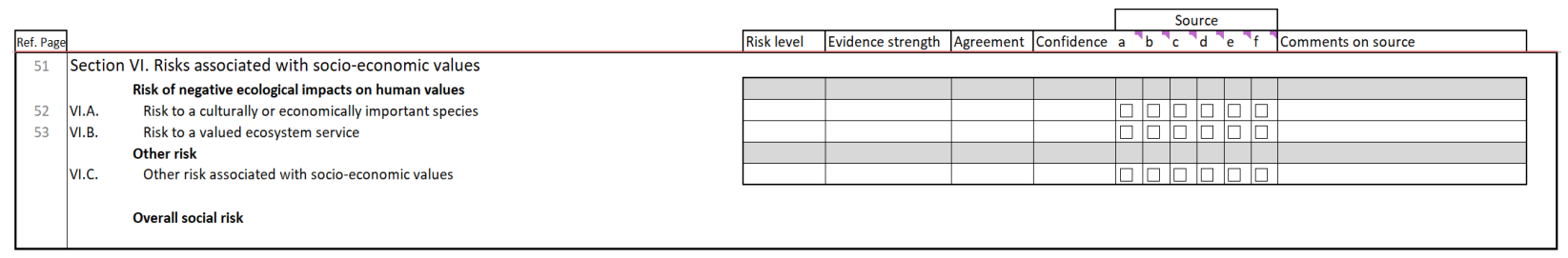

Figure 10. Screen capture of Section VI of the managed relocation risk assessment protocol presented in a simple spreadsheet format. Spreadsheet rows contain section names and risk criteria. Columns for risk level, evidence strength, agreement, and confidence contain drop down lists for ranking selection.

Separate columns with checkboxes provided for sources of evidence allow users to select multiple sources. Page numbers presented next to each criterion direct users to where guidance for each criterion is found in these protocols. Interactive spreadsheet tool found at https://irma.nps.gov/DataStore/Reference/Profile/2280035.

\section{VI.A. Risk to a Culturally or Economically Important Species}

This subsection incorporates the risk addressed in the previous sections and applies it specifically to culturally, socially, or economically important ('important' hereafter, means a species deemed important by a human value) species within the recipient and surrounding ecosystems. In some cases, this section may seem redundant of the previous sections. But here, weight is given to species that are particularly important to people.

Will the target negatively impact any societally valued species?

- Through any of the interactions discussed in the previous sub-sections, will the target change the abundance of a species such that the use (economic or aesthetic) or extraction of this species would decline?

\section{Risk Scoring}

We consider risk here to be a function of biological interactions between the target and the societally valued species under consideration. The risks described below are not exhaustive of the ways in which an important species may be impacted by the relocated target.

Low. The target does not directly interact with any societally valued species, nor are there any known indirect impacts of the target on a societally valued species.

Moderate. The target competes with or consumes a societally valued species, though it is unlikely to reduce the abundance or fitness of that species. The target may alter the behavior of a societally valued species such that it can no longer be used or extracted at the current rate.

High. The target competes with or consumes a societally valued species and may result in a reduction in the abundance or fitness of that species. The target carries a disease that may be transmittable to a societally valued species, but that disease can be detected and treated.

Very high. The target directly interacts with a societally valued species and will likely result in a reduction in the abundance or fitness of that species, and lead to a reduction in the current rate of 
use or extraction of that species. The target is a known carrier of a disease that may be transmittable to a societally valued species for which complete treatment is not likely.

Not applicable. There are no widely societally valued species within the recipient ecosystem.

\section{VI.B. Risk to a Valued Ecosystem Service}

This subsection addresses how a target may negatively affect a service provided by the recipient ecosystem. This may include an ecosystem service within the recipient ecosystem or originating within and providing benefits beyond the recipient ecosystem. This section does not seek to valuate any ecosystem services provided by the recipient ecosystem, but simply to highlight if they exist and would be at risk due to the managed relocation. Ecosystem services may include supporting animal populations that reduce agricultural pests, contributions to clean water, carbon or nitrogen fixation. If for example, the valued ecosystem service was the delivery of clean water from a stream flowing through a forested area, the relocation of a highly fire-supportive species could increase fire severity and negatively impact water quality through post-fire run-off; this would constitute a high or very high risk to the valued ecosystem service.

Would the target disrupt any ecosystem services in the recipient ecosystem?

\section{$\underline{\text { Risk Scoring }}$}

Low. The recipient ecosystem provides an ecosystem service but is unlikely to be affected by the target.

Moderate. The recipient ecosystem provides an ecosystem service related to the functional role of the target.

High. The recipient ecosystem provides an ecosystem service that may be compromised by the target.

Very high. The recipient ecosystem provides an ecosystem service that will be completely disrupted by the addition of the target.

Not applicable. The recipient ecosystem does not provide any ecosystem services. 


\section{Reaching a Decision}

Having now completed the risk assessment spreadsheet, a decision must be reached. The scoring of risk does not simply point to a decision. Further, we do not recommend summing scores to create an overall composite score. However, summarizing the risk scores is necessary to complete the picture of risk. We recommend using a figure that colors boxes in accordance to risk (Figure 11).

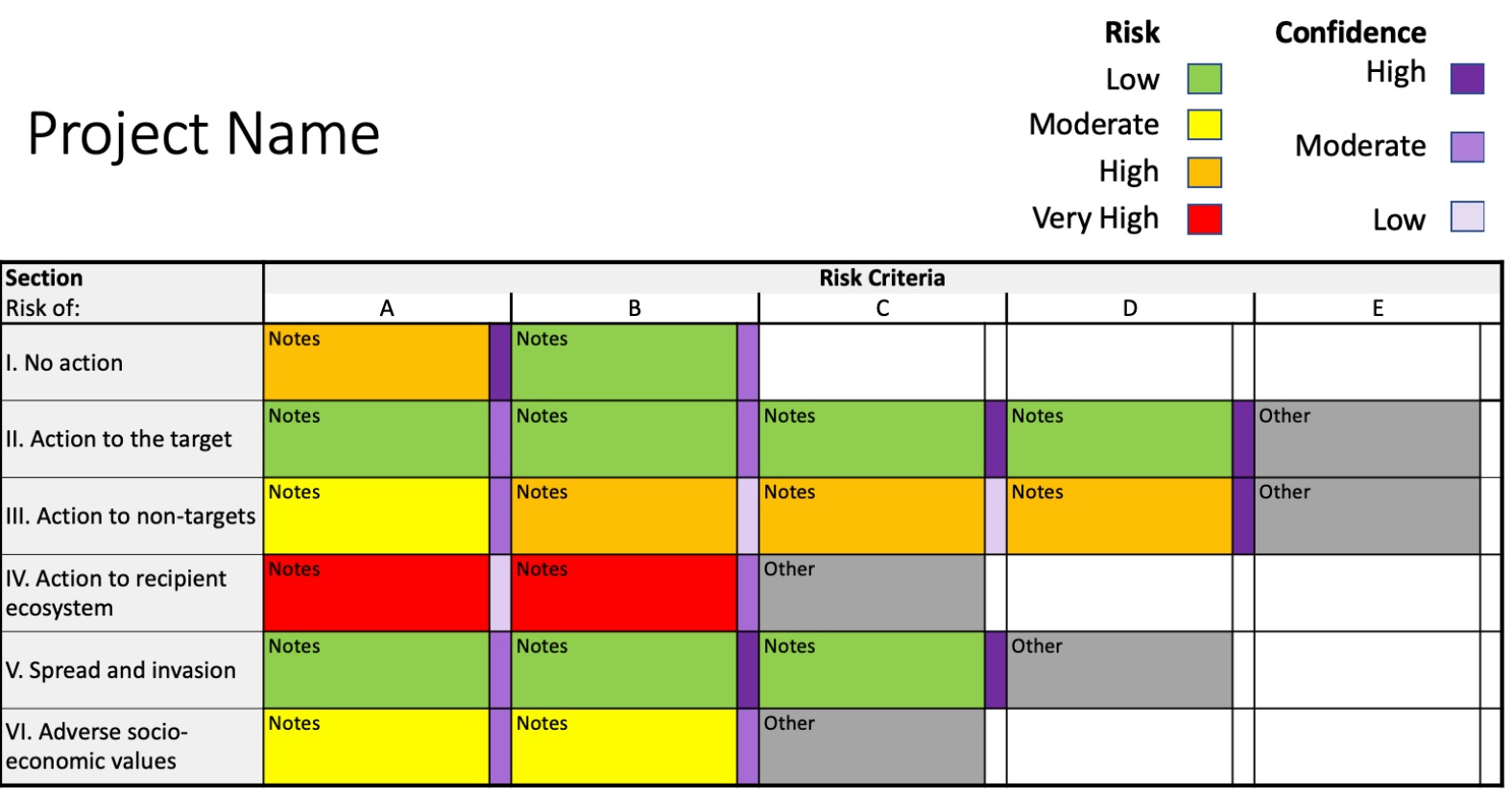

Figure 11. Schematic table of a visual risk summary where 17 boxes represent the individual risk assessments within the six risk sections (rows) and specific criteria as described above (columns). Boxes are color coded for assessed risk from green (low risk) to red (very high risk). Narrow colored rectangles to the right represent the scoring of confidence where deep purple represents high confidence and light purple represents low confidence. Additional boxes, in gray, are for other attributes that may be assessed depending on specific conditions of the proposed project. Text within boxes is used to capture shorthand risk assessment notes.

The decision to move forward in considering managed relocation requires evaluating the risk scores in at least three major ways that we describe below. We recommend using this visual summary as a focal illustration with which to discuss and then make a decision.

Acceptability of overall uncertainty in risk. Does the overall level of uncertainty in evaluating risk exceed some level of unacceptability? If so, the urgency for action must be weighed with the potential to reduce this uncertainty with new research. In some cases, it is unlikely that more time will allow for a reduction in uncertainty; for example, anticipating evolutionary changes in a target or non-targets will always be difficult. Therefore, when making a decision, the decisionmaker must decide if it is acceptable to move forward with an action even with some high level of uncertainty. 
Acceptability of overall levels of risk. Does the overall level of risk suggest that there is an unacceptably high likelihood that one of the risk factors causes an unintended and unwanted outcome? There is no objective way to decide whether the level of risk is unacceptably high. The risk that may be accepted in a relocation decision should be balanced against the risk to the target species or recipient ecosystem by not taking an action. We suggest visually assessing the distribution of risk scores; not to create an average risk score. Higher overall levels of risk may tilt the balance toward rejection of a proposal to engage in managed relocation. High levels of risk associated with high uncertainty should, at a minimum, initiate a slow, deliberative process of evaluating uncertain risk factors that are viewed as potentially important.

Acceptability of risk in a critical area. Overall levels of uncertainty and risk may be viewed as acceptable, yet there may still be a decision not to move a species. This would be the case if there is some singular aspect of risk that is deemed to be high. For example, if managed relocation may result in a very high risk of transmitting a disease to a closely related, and endangered, species, then the decision-maker might decide not to proceed despite other aspects of risk being considered low. The target type may also factor into this decision. For example, if risk to the relocated individuals is very high because very few individuals would survive the move, and the relocated individuals are seeds, then a manager may choose to move forward with the action. However, the same decision might not be reached if the relocated individuals were breeding adult vertebrates.

Ecological justification, feasibility, management priority, social acceptability, and ecological risk represent critical factors to justify, or not, managed relocation of species for conservation (Figure 2). None of these aspects is likely to return an assessment that is all 'yes' or all 'no'. Each requires assessment along a gradient of importance, priority or acceptability. Further, each attribute exists in a constantly changing landscape of opportunities and costs, and thus requires constant assessment attention. For example, it may be very difficult to assess social acceptability of a proposed managed relocation project without sharing the results of this assessment of risk.

If, after an initial assessment, each of these five criteria points toward moving forward with a proposed project, then there remains further work to develop an implementation plan. Implementation plans have logistical components, which is beyond the scope of these risk assessment protocols. There are, however, choices to be made that may drive ecological risk. For example, risks may vary depending on how individuals are extracted from existing populations, how individuals are cared for in transition, how they are released, where they are released, when they are extracted and released, and post-release management strategy. We recommend revisiting the risk protocols to subsequently score the risk of different particular strategies to engage in managed relocation.

\section{Multi-Criteria Action Assessment}

These protocols were designed to inform the manager's decision on the binary choice of whether or not to engage in managed relocation. If the answer derived from using the protocols is that the risk is within reason, then numerous other critical decisions remain (Figure 2). Assuming that managers move forward with strategic planning for a managed relocation action, managers will generally have 
more decisions and may want to minimize risks that vary across these action options. Choices may include the location and number of individuals that are selected from the source population, husbandry of the population before re-release, or specifics associated with the recipient location (Table 3).

Table 3. Representative (hypothetical) decision issues that could be used to create alternative action scenarios for a managed relocation project.

\begin{tabular}{ll}
\hline Decision Category & Decision Issue \\
\hline Target & - Number of individuals collected \\
- Number and source of populations from which individuals are collected \\
- Attributes of individuals selected for relocation (e.g., genetics, breeding status) \\
- Timing and sequence of removing individuals from a population \\
- Different release strategies \\
- Status of the target with respect to the Endangered Species Act \\
- Quarantine or other measures to assure transference of disease-free individuals \\
- Assessment of potential 'hitch-hiking' organisms (pests and parasites) to be novel \\
- species with potential adverse impacts to the recipient location \\
- Captive breeding \\
- Number and location of recipient ecosystem sites \\
- Proximity of recipient ecosystem(s) to potential habitats in different ecosystems \\
- Ecosystem condition, community structure \\
- - Potential to become a harmful invasive species \\
- Timing and sequence of adding individuals of the target \\
- Risk of hybridization with closely related species \\
- Presence of threatened or endangered species ${ }^{13}$ \\
- External stressors
\end{tabular}

The protocols provided here can also be used to evaluate alternative scenarios against each other in order to choose the best action alternative (Figure 2). For example, alternative actions may trade off the probability of success with risk to the recipient ecosystem by moving different numbers of individuals from different source populations. It is preferable to choose actions that both reduce financial and social costs, and also those actions that reduce ecological risk.

\footnotetext{
${ }^{13}$ We do not limit the use of the terms "endangered" and "threatened" to species defined as such by listing under the Endangered Species Act, but instead mean it in the broader sense of "at risk of extinction" by some criteria.
} 
Managers will typically consider more than one combination of options. Thus far, the risk assessment strategy we present here does not consider comparing risk across a suite of potential actions. Yet, this is exactly what a manager must do when making a strategic plan of action.

Taking action requires, among other things, choosing the option that is likely to provide the best return on the investment. In this case, we might seek to find the management option with the highest likelihood of success while minimizing both costs and the risks of unintended negative consequences (Joseph et al. 2009). A simple, but formal, way to optimize across action alternatives is to compare estimated action values across of a set of action scenarios (strategic combinations of choice alternatives).

$$
\text { Action } \text { Value }_{\mathrm{i}}=\left(P(\text { success })_{\mathrm{i}} *\left(F\left(\text { benefit }_{\mathrm{i}}\right)\right) / \text { cost }_{\mathrm{i}}\right.
$$

Where $P$ (success) is the estimated probability ( 0 to 1 ) of success of the action to achieve a desired outcome; $F$ (benefit) is a benefit function to estimate, for example, how much extinction risk is reduced by taking the action; Cost is considered broadly to include monetary costs (some may be more expensive than others), social costs (some action alternatives may be more socially acceptable than others) and costs associated with ecological risk (some action scenarios may result in greater or lesser chance of harming the recipient ecosystem); and $i$ is an index that represents each proposed alternative action scenario. We recognize that the terms 'success', 'benefit' and 'cost' are each poorly specified in this simple equation. Operationalizing this into an actual multi-criteria assessment requires explicit specification of these terms. For example, 'benefit' could be defined as a quantitative reduction in the risk of extinction of a threatened species (as above), or a delayed time to predicted extinction. 


\section{Literature Cited}

Baker, H. G. 1965. Characteristics and modes of origin of weeds. Pages 147-172 in H. G. Baker and G. L. Stebbins, editors. The genetics of colonizing species. Academic Press, New York.

Baron, J. S., L. Gunderson, C. D. Allen, E. Fleishman, D. McKenzie, L.A. Meyerson, J. Oropeza, and N. Stephenson. 2009. Options for National Parks and reserves for adapting to climatic change. Environmental Management 44:1033-1042.

Barratt, B. I. P., F. G. Howarth, T. M. Withers, J. M. Kean, and G. S. Ridley. 2010. Progress in risk assessment for classical biological control. Biological Control 52(3):245-254.

Barratt, B. I. P., V. C. Moran, F. Bigler, and J. C. van Lenteren. 2018. The status of biological control and recommendations for improving uptake for the future. BioControl 63: 155-167.

Bennett, N. J., R. Roth, S. C. Klain, K. Chan, P. Christie, D. A. Clark, G. Cullman, D. Curran, T. J. Durbin, G. Epstein, A. Greenberg, M. P. Nelson, J. Sandlos, R. Stedman, T. L. Teel, R. Thomas, D. Veríssimo, and C. Wyborn. 2017. Conservation social science: understanding and integrating human dimensions to improve conservation. Biological Conservation 205:93-108.

Burgman, M. 2009. Risks and decisions for conservation and environmental management. Cambridge University Press. https://doi.org.10.1017/CBO9780511614279.

Cole, K.L., K. Ironside, J. Eischeid, G. Garfin, P.B. Duffy, C. Toney. 2011. Past and ongoing shifts in Joshua tree distribution support future modeled range contraction. Ecol Appl 21(1): 137-149.

Conner, A.J. Glare, T.R., Nap, J-P. 2003. The release of genetically modified crops into the environment. The Plant Journal 33:19-46.

Environmental Protection Agency (EPA). 1998. Guidelines for ecological risk assessment. Federal Register 63(93):26846-26924.

Fair, J. M., C. D. Hathcook, and A. W. Bartlow. 2018. Avian communities are decreasing with piñon pine mortality in the southwest. Biological Conservation 226:186-195.

Foden, W. B., and B. E. Young, editors. 2016. IUCN SSC guidelines for assessing species' vulnerability to climate change. Version 1.0. Occasional Paper of the IUCN Species Survival Commission No. 59. IUCN Species Survival Commission, Cambridge, UK and Gland, Switzerland.

Fraser, D. J. 2009. How well can adaptive captive breeding programs conserve diversity? A review of salmonids. Evolutionary Applications 1:535-586.

Glick, P., B. A. Stein, and N.A. Edelson, editors. 2011. Scanning the conservation horizon: a guide to climate change vulnerability assessment. National Wildlife Federation, Washington, D.C. 
Gregory, A. J., R. S. A. Kaler, and T. J. Prebyl. 2012. Influence of translocation strategy and mating system on the genetic structure of a newly established population of island ptarmigan.

Conservation Genetics 13:465-474.

Hayes, K. R., and S. C. Barry. 2008. Are there any consistent predictors of invasion success? Biological Invasions 10:483-506.

Hemming V., M. A. Burgman, A. M. Hanea, M. F. McBride, and B. C. Wintle. 2018. A practical guide to structured expert elicitation using the IDEA protocol. Methods in Ecology and Evolution 9:169-180.

Intergovernmental Panel on Climate Change (IPCC). 2013. Climate change 2013: the physical science basis. Cambridge University Press, Cambridge, UK.

Invasive Species Advisory Committee (ISAC). 2017. Managed relocation reducing the risk of biological invasion. Invasive Species Advisory Committee, Washington, D.C.

International Union for the Conservation of Nature - Species Survival Commission (IUCN/SSC). 2013. Guidelines for reintroductions and other conservation translocations. Version 1.0. IUCN Species Survival Commission, Gland, Switzerland.

International Union for the Conservation of Nature (IUCN). 2017a. The IUCN Red List of Threatened Species. Version 2017-3. Available at: http://www.iucnredlist.org.

International Union for the Conservation of Nature (IUCN). 2017b. Consultation document: IUCN standard classification of the impact of invasive alien taxa. Version 1. IUCN, Gland, Switzerland.

Jeschke, J. M., S. Bacher, T. M. Blackburn, J. T. A. Dick, F. Essl, T. Evans, M. Gaertner, P. E. Hulme, I. Kühn, A. Mrugała, J. Pergl, P. Pyšek, W. Rabitsch, A. Ricciardi, D. M. Richardson, A. Sendek, M. Vilà, M. Winter, and S. Kumschick. 2014. Defining the impact of non-native species. Conservation Biology 28:1188-1194.

Joseph, L.N., R.F. Maloney, and H.P. Possingham. 2009. Optimal allocation of resources among threatened species: a project prioritization protocol. Conservation Biology 23:328-338.

Kostyack J., J. J. Lawler, D. D. Goble, J. D. Olden, and J. M. Scott. 2011. Beyond reserves and corridors: policy solutions to facilitate the movement of plants and animals in a changing climate. Bioscience 61:713-719.

Larson, E.R., and J.D. Olden. 2012. Using avatar species to model the potential distribution of emerging invaders. Global Ecology and Biogeography 21:1114-1125.

Lawler, J. J., and J. D. Olden. 2011. Reframing the debate over assisted colonization. Frontiers in Ecology and Evolution 9(10):569-574.

Lee, E., P. McCormack, P. Michael, S. W. Molloy, E. Cowan, T. Mustonen, and H. Possingham. 2016. The language of science: essential ingredients for indigenous participation. [square 
brackets]: CBD Newsletter for Civil Society 10:22. Available at: https://www.cbd.int/ngo/squarebrackets/square-brackets-2016-04-en.pdf.

Lockwood, J. L., M. F. Hoopes, and M. P. Marchetti. 2013. Invasion ecology. Wiley-Blackwell Publishing, Sussex, UK.

Marchetti, M. P., P. B. Moyle, and R. Levine. 2004. Invasive species profiling? Exploring the characteristics of non-native fishes across invasion stages in California. Freshwater Biology 49(5):646-661.

Mawdsley, J. R., R. O’Malley, and D. Ojima. 2009. A review of climate change adaptation strategies for wildlife management and biodiversity conservation. Conservation Biology 23:1080-1089.

McCormack, P.C. 2018. Conservation introductions for biological adaptation under climate change. Transnational Environmental Law 7(2):323-345.

McLachlan, J. S., J. J. Hellmann, and M. W. Schwartz. 2007. A framework for debate of assisted migration in an era of climate change. Conservation Biology, 21(2):297-302.

Millar, C. L., and N. L. Stephenson. 2015. Temperate forest health in an era of mega-disturbance. Science 349:823-826.

National Park Service (NPS). 2006. Management policies 2006. US Department of the Interior, National Park Service, Washington, DC.

O’Donnell, R. P., C. A. Crost, and K. E. Mock. 2017. Cryptic invasion of northern leopard frog (Rana pipiens) across phylogenetic boundaries and a dilemma for conservation of a declining amphibian. Biological Invasions 19:1039-1052.

Olden, J. D., M. J. Kennard, J. J. Lawler, and L. N. Poff. 2011. Challenges and opportunities in implementing managed relocation for conservation of freshwater species. Conservation Biology 25:40-47.

Palmer, M.A., D.P. Lettenmaier, N.L. Poff, S.L. Postel, B. Richterand R. Warner. 2009. Climate change and river ecosystems: protection and adaptation options. Environmental Management 44: 1053-1068.

Reed, R. N., and G. H. Rodda. 2009. Giant constrictors: biological and management profiles and an establishment risk assessment for nine large species of pythons, anacondas, and the boa constrictor: U.S. Geological Survey Open-File Report 2009-1202, 302 p.

Resnik, J. R. 2018. Biodiversity under siege, invasive animals and the National Park Service: a state of the knowledge report. Natural Resource Report NPS/NRSS/BRD/NRR-2018/1679. National Park Service, Fort Collins, Colorado.

Ricciardi, A., and D. Simberloff. 2009. Assisted colonization is not a viable conservation strategy. Trends in Ecology and Evolution 24:248-253. 
Richardson, D. M., J. J. Hellmann, J. S. McLachlan, D. F. Sax, M. W. Schwartz, P. Gonzalez, E. J. Brennan, A. Camacho, T. L. Root, O. E. Sala, S. H. Schneider, D. M. Ashe, J. R. Clark, R. Early, J. R. Etterson, E. D. Fielder, J. L. Gill, B. A. Minteer, S. Polasky, H. D. Safford, A. R. Thompson, and M. Villend. 2009. Multidimensional evaluation of managed relocation. Proceedings of the National Academy of Sciences 106(24):9721-9724.

Robinson, K. F., A. K. Fuller, R. C. Stedman, W. F. Siemer, and D. J. Decker. 2019. Integration of social and ecological sciences for natural resource decision making: challenges and opportunities. Environmental Management 63:565-573.

Roy, J. 1990. In search of the characteristics of plant invaders. Pages 335-352 in: F. di Castri, A. J. Hansen, and M. Debussche, editors. Biological invasions in Europe and the Mediterranean Basin. Kluwer Academic Publishers, Norwell, Massachusetts.

Roy, H.E., W. Rabitsch, R. Scalera, A. Stewart, B. Gallardo, P. Genovesi, F. Essl, T. Adriaens, S. Bacher, O. Booy, E. Branquart, S. Brunel, G. H. Copp, H. Dean, B. D’hondt, M. Josefsson, M. Kenis, M. Kettunen, M. Linnamagi, F. Lucy, A. Martinou, N. Moore, W. Nentwig, A. Nieto, J. Pergi, J. Peyton, A. Roques, S. Schindler, K. Schonrogge, W. Solarz, P. D. Stebbing, T. Trichkova, S. Vanderhoeven, J. van Valkenburg, A. Zenetos. 2017. Developing a framework of minimum standards for the risk assessment of alien species. Journal of Applied Ecology 55:526538.

Salafsky, N., D. Salzer, A. J. Stattersfield, C. Hilton-Taylor, R. Neugarten, S. H. Butchart, B. Collen, N. Cox, L. L. Master, S. O'Connor, and D. Wilkie. 2008. A standard lexicon for biodiversity conservation: unified classifications of threats and actions. Conservation Biology 22(4):897-911.

Sakai, A. K., F. W. Allendorf, J. S. Holt, D. M. Lodge, J. Molofsky, K. A. With, S. Baughman, R. J. Cabin, J. E. Cohen, N. C. Ellstrand, D. E. McCauley, P. O'Neil, I. M. Parker, J. N. Thompson, and S. G. Weller. 2001. The population biology of invasive species. Annual Review of Ecology and Systematics 32(1):305-332.

Schwartz, M. W., J. J. Hellmann, J. M. McLachlan, D. F. Sax, and 28 others. 2012. Managed relocation: integrating the scientific, regulatory and ethical challenges. Bioscience 62:732-743.

Stein, B. A., P. Glick, N. Edelson, and A. Staudt, editors. 2014. Climate-smart conservation: putting adaptation principles into practice. National Wildlife Federation, Washington, D.C.

Suter, G.W. II. 2016. Ecological Risk Assessment. Second Edition. CRC Press. 624 p.

U.S. Department of Agriculture (USDA). 2019. Guidelines for the USDA-APHIS-PPQ weed risk assessment process. Technical Procedure E-300, Version 2.3.

U.S. Fish and Wildlife Service (USFWS). 2018. Standard operating procedures for the rapid screening of species' risk of establishment and impact in the United States. fws.gov/injuriouswildlife/pdf_files/ERSS-SOP-Final-Version.pdf 
United Nations Environment Programme (UNEP). 2019. Global environment outlook - 6. Cambridge University Press, Cambridge, UK.

Walker, L. R., and E. A. Powell. 1999. Regeneration of the Mauna Kea silversword Argyroxiphium sandwicense (Asteraceae) in Hawaii. Biological Conservation 89:61-70.

Wallingford, P. D., T. L. Morelli, et al. In Revision. Assessing impacts of climate-driven range shifts through the lens of invasion biology. Nature Climate Change.

Weber, E. P., J. M. Belsky, D. Lach, and A. S. Cheng. 2014. The value of practice-based knowledge. Society and Natural Resources 27(10):1074-1088.

Williams, B. K., R. C. Szaro, and C. D. Shapiro. 2009. Adaptive management: The U.S. Department of the Interior technical guide. Adaptive Management Working Group, U.S. Department of the Interior, Washington, DC.

Zimmerman, H. G., V. C. Moran, and J. H. Hoffmann. 2001. The renowned cactus moth, Cactoblastis cactorum (Lepidoptera: Pyralidae): Its natural history and threat to native Opuntia floras in Mexico and the United States of America. Florida Entomologist 84(4):543-551. 


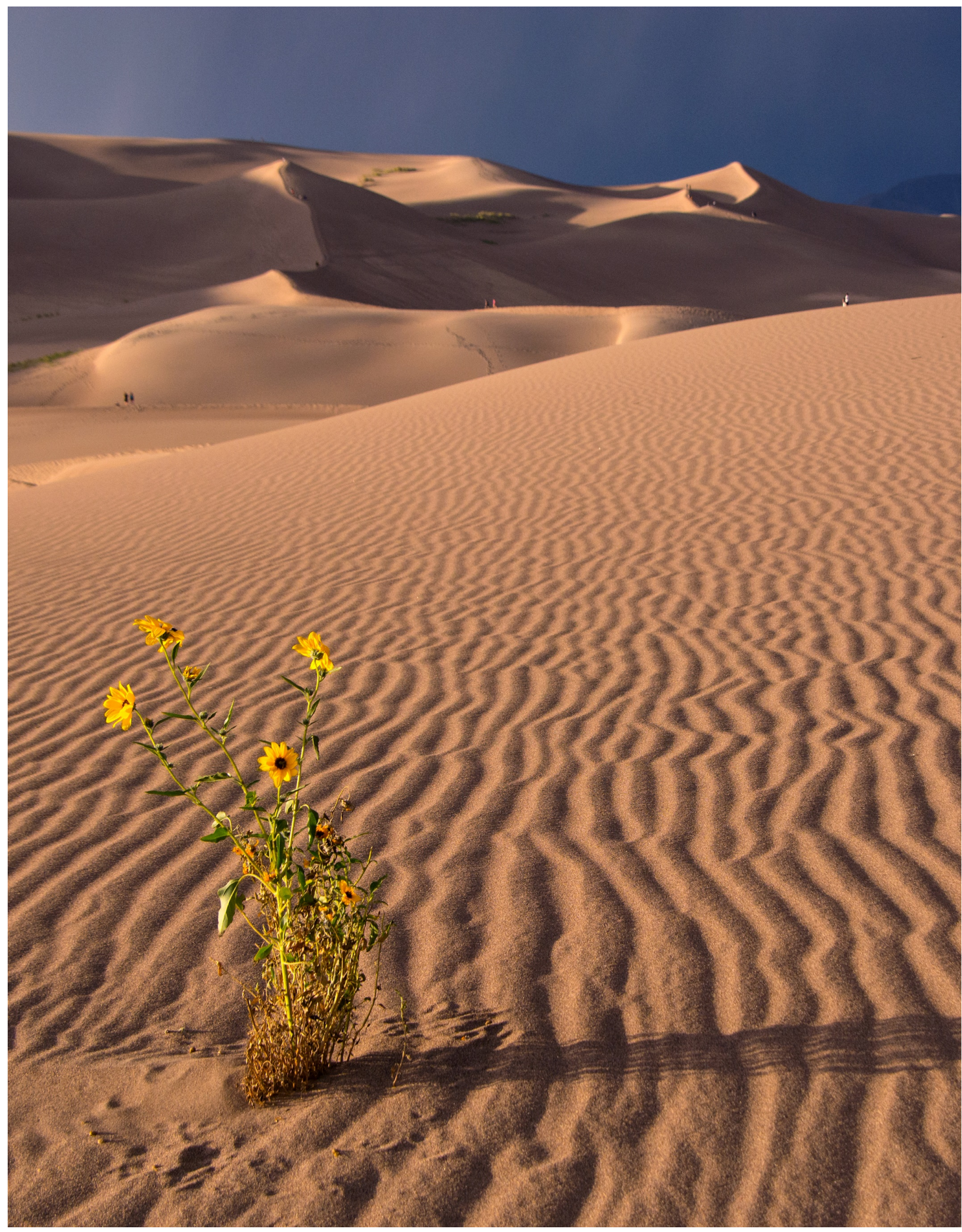

A sunflower grows in Great Sand Dunes National Park. Credit: P. Myers, NPS 


\section{Appendix A. Definitions}

Allee effect. Reduced population growth at a small population size or density.

Assisted migration. The intentional movement of a conservation target outside its historical range, often in response to climate change. Can be used to avoid extirpation of the target or to replace a declining function in the recipient ecosystem. [synonyms: assisted dispersal, assisted colonization, managed relocation]

Biologically extinct. As compared with functionally extinct, biologically extinct refers to the complete disappearance of a species.

Character displacement. Differences in characteristics of two similar species are enhanced where those species overlap geographically.

Conservation translocation. The intentional movement of a target where the primary objective is a conservation benefit.

Ecological replacement. Relocation of a species outside of its historical range to perform a specific ecological function following the extirpation of the original species that performed that function.

Ecological risk assessment. Evaluates the likelihood that adverse ecological effects may occur or are occurring as a result of exposure to one or more stressors (EPA 1998).

Endangered. We do not limit the use of this term to species defined as such by listing under the Endangered Species Act, but instead mean it in the broader sense of "at risk of extinction" by some criteria, and at greater risk than a threatened species.

Functionally Extinct. A species falling to sufficiently low numbers that it no longer provides ecosystem services for which it is valued. Hence, we may be concerned with biological extinction of species, but in managing ecosystem functionality, we may be interested in functions such as predators acting as an effective control of herbivore populations or plants reducing erosion potential.

Genetic Fitness. Can cover a broad range of issues and is often poorly defined. Here we reference genetic fitness as it relates to the genetic diversity of a target that supports persistence of the target. Reduced genetic fitness would therefor refer to low genetic variability that reduces persistence potential.

Historical range. The geographic area where a species was known to occupy (as opposed to modeled, or hypothetical occupancy) in the past. Here we recognize an implicit reference to a baseline condition which may differ slightly depending on the specific case study. [synonym used by NPS: natural range].

Introduction. The intentional movement of an organism beyond its historical range.

Invasive species.

- Biological definition (used in Section V, based on Lockwood et al. 2013). A species that has spread, usually aggressively, within its ecosystem and/or into a new ecosystem, and results in negative ecological impact. The term may be applied to either native or non-native species.

- Federal definition (from Exec. Order 13751). With regard to a particular ecosystem, a nonnative organism whose introduction causes or is likely to cause economic or environmental harm, or harm to human, animal, or plant health. 
Note: in both definitions not all non-native species are considered invasive. The biological definition focuses on ecological impact while the federal definition includes a wider array of impacts.

Managed relocation. The intentional movement of a species beyond its historical range. We treat managed relocation to be equivalent to assisted migration or assisted colonization and a subset of the broader set of actions that may be considered conservation translocations.

Non-native species. Species that occupy, or could, occupy natural lands directly or indirectly as the result of deliberate or accidental human activities.

Non-target species. All taxa other than the target. Here used specifically to refer to non-focal species in recipient ecosystems.

Recipient ecosystem. The area receiving the relocated target.

Reinforcement. The intentional movement of a target inside its historical range to supplement an existing population of conspecifics.

Reintroduction. An often mis-used term that technically only refers to the replacement of an introduced species to a location (e.g., putting goats back onto Santa Cruz island). More frequently used to refer to the intentional movement of a target inside its historical range from which is has disappeared. [synonyms: restoration, population restoration]

Relocation. The intentional, human-mediated movement of a target to an area outside its historical range.

Risk Assessment. Systematic process of evaluating potential risks involved in a proposed action.

Source population. The population of the target species from which individuals will be collected for translocation.

Target. The species, subspecies, population or taxon to be relocated.

Threatened. We do not limit the use of this term to species defined as such by listing under the Endangered Species Act, but instead mean it in the broader sense of "at risk of extinction" by some criteria, and less at risk than an endangered species.

Translocation. The intentional, human-mediated movement of a target from one area into another within its historical range.

Wild. Any location dominated by natural vegetation cover types and species are not caged or specifically managed (e.g., fed or gardened). 


\section{Appendix B. Management Use Examples}

A series of six mostly hypothetical use examples are used to describe when it is, and when it is not, advised to use these ecological risk assessment protocols.

\section{1) Species Protection}

a) Climate change threatens a species with extinction within its historical range, within a park.

i) Details. The proposal is to shift the distribution of the target species upslope, within a park, to conserve viable populations for visitor enjoyment and species protection.

ii) Example. Giant sequoia in Sequoia, Kings Canyon or Yosemite National Parks. Bull trout in Glacier National Park.

iii) Appropriateness. This is a focal problem envisioned for this risk assessment strategy.

b) Climate change threatens a species with extinction within a park, with proposed recipient sites outside the park.

i) Details. A proposal is made to use individuals from within the park to seed new populations outside the park in places where new populations may thrive.

ii) Example. Joshua tree in Joshua Tree National Park may have difficulty maintaining populations within the park. We imagine a proposal to use seed or trees from the park to establish new populations in cooler climates upslope and north of the park.

iii) Appropriateness. This is a focal problem envisioned for this risk assessment strategy.

c) Climate change threatens a species with extinction within its historical range, outside a park, with proposed recipient locations within a park and outside the historical range.

i) Details. The proposal is to shift the distribution of the target species upslope or northward and into a national park to conserve viable populations.

ii) Moving a northern Sierra endangered plant species uphill to Yosemite or Sequoia and Kings Canyon National Parks.

iii) Appropriateness. This is a focal problem envisioned for this risk assessment strategy.

\section{2) Species Restoration or Reinforcement}

a) Restoring a species to locations where it formerly occurred.

i) Details. A proposed restoration or reinforcement of declining populations into a location where a species formerly existed. The purpose of this action could be to reinforce and support the target population, or to increase ecosystem functionality.

ii) Example. Moving gray wolves back into Yellowstone.

iii) Appropriateness. Although the protocols could be used to assess risk if the managers think it appropriate, this is not the primary purpose of this risk assessment. We assume that the risk of restoring a species to a location in which it formerly occurred would pose a substantially reduced ecological risk (NPS 2006).

\section{3) Guiding Ecosystem Change}

a) Climate change threatens an ecosystem within a park.

i) Details. A proposed management action that recommends transitioning ecosystems to new more ecologically resilient ones, which entails bringing species from outside the 
park, beyond their historical ranges, into the park to change the ecosystem composition, structure and/or function of some portion of the park.

ii) Example. Managing to encourage boreal forest colonization of tundra in formerly tundra ecosystems as climates warm.

iii) Appropriateness. This is not the focal problem envisioned for these protocols. This entails a manager needing adaptation strategies for broadscale ecosystem change, which is outside our scope.

b) Improving changing ecosystem with managed relocation of a key missing species.

i) Details. Climate change is changing ecosystems, so management proposes to introduce a key component of the new ecosystem that has not self-dispersed, thus seeking to improve the emerging ecosystem with the managed relocation of a missing species.

ii) Example. Moving moose into newly forming boreal forests within a park.

iii) Appropriateness. Though this entails moving a species that is, itself, not at risk of extinction, this problem still focuses on the relocation of a single target. Therefore, this is a focal problem envisioned for these protocols. The purpose of the managed relocation would be for the benefit of the emerging ecosystem. 


\section{Appendix C. Risk Assessment Case Studies}

The following are four case studies completed by various sub-sets of the team of universityassociated researchers. These are hypothetical cases; although, for all but Pitcher's thistle, we have reports from NPS staff that the discussion of whether or not to relocate these species has been discussed at some level. With the exception of bull trout where an action has been taken, the scenarios are hypothetical. The cases vary in depth and application. This is intentional in that the main purpose was for the focal team to think through the utility and ease of use of the spreadsheet to apply the risk assessment protocols by applying it to cases. We did not attempt to standardize across uses because we expect that actual uses of these protocols would also vary in depth and detail. Since these were all done in a reasonably short period of time, with specific funding resources, no formal expert elicitation was used. Experts were informally consulted, particularly with the Karner blue butterfly. In no case do we recommend actually using these assessments for informing a decision.

\section{Case Study: Risk Assessment of the Managed Relocation of Bull Trout (Salvelinus confluentus)}

Julian Olden (University of Washington) and David Lawrence (National Park Service)

\section{Hypothetical Action}

We completed a risk assessment of the managed relocation of bull trout, Salvelinus confluentus (Suckley 1859; Figure C-1). Bull trout is currently listed as "Threatened" in the United States under the Endangered Species Act, ranked "Of Special Concern" or "Threatened" for three of four geographic populations by the Committee on the Status of Endangered Wildlife in Canada, and is considered globally "Vulnerable" according to the IUCN Red List. For this case study, we provide information that allows for a broad evaluation of a generic managed relocation to a new location, as well as making specific reference to a published evaluation of the relocation of three bull trout populations into historically unoccupied habitats within their natal drainages in Glacier National Park, Montana. The former is based on a broad body of peer-reviewed literature, whereas the latter is informed by information provided in Galloway et al. (2016) and other associated publications.

Glacier National Park supports a significant proportion of the remaining lake habitat within bull trout's range. The vast majority of adfluvial bull trout populations in western Glacier National Park have dramatically declined over the last three decades due to the invasion and establishment of nonnative lake trout, Salvelinus namaycush, and habitat degradation occurring because of a changing climate (Fredenberg 2002; GNP et al. 2003). Where they have been introduced, lake trout consistently displace native bull trout through predation and competition (Martinez et al. 2009). In lakes for which data exist, bull trout populations have declined to the point of functional extirpation (Fredenberg 2002; D’Angelo and Muhlfeld 2013; Downs et al. 2013, 2015). 


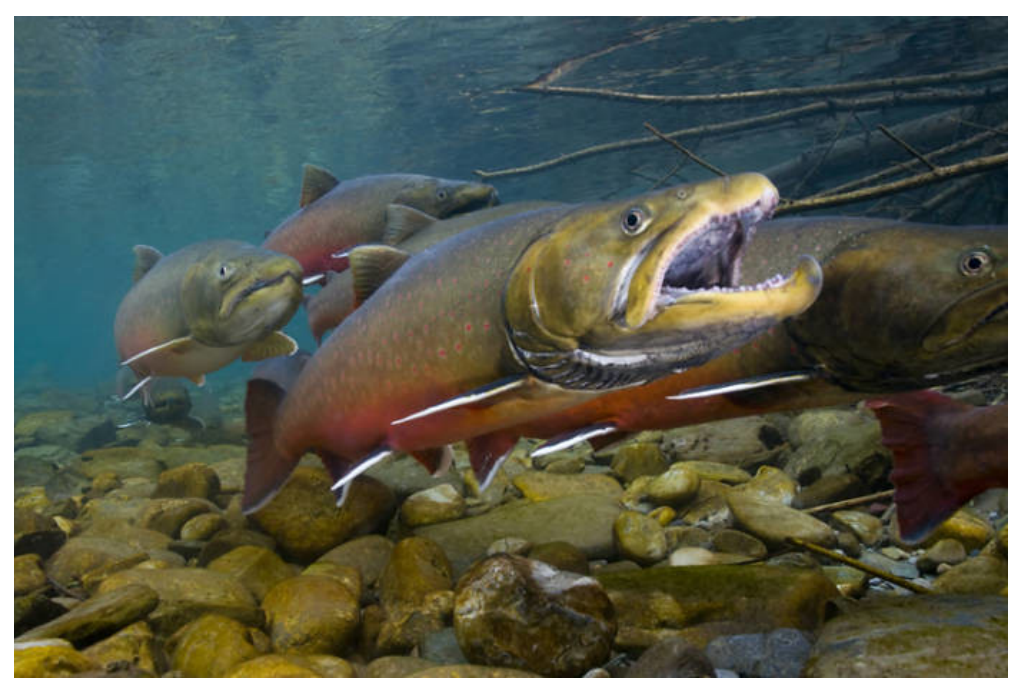

Figure C-1. The bull trout is a char of the family Salmonidae and is native to northwestern North America. Photo credit: USFWS

The Montana Cooperative Fishery Research Unit, USGS, NPS, and USFWS conducted an assessment of bull trout relocation potential in Glacier National Park (Galloway 2014; Galloway et al. 2016). Potential recipient sites in Glacier National Park were evaluated using a scoring framework that included the ability of the site to support relocated fish, possible negative impacts of a relocation on native aquatic biota, and the availability of within-basin donors (USFWS 2005; Galloway 2014; Galloway et al. 2016). Based on this assessment, managers have already conducted some relocations of bull trout above natural barriers within their natal drainages to conserve and maintain localized ecological and evolutionary processes in the presence of invasive species. The study sites chosen by managers were located in isolated stream-and-lake networks within the Logging, Camas, and Lincoln Creek drainages of western Glacier National Park (Figure C-2.). These potential recipient sites were selected because (1) bull trout populations exist downstream of these sites, providing the possibility of within-drainage relocation; (2) the sites are isolated above natural barriers (e.g., waterfalls) to fish migration, thus preventing future invasions by lake trout and other nonnative species; and (3) that these sites offer physical and biological conditions that may be suitable for bull trout persistence. However, these three sites differ in numerous key attributes, ultimately resulting in different levels of risk as discussed more below. 


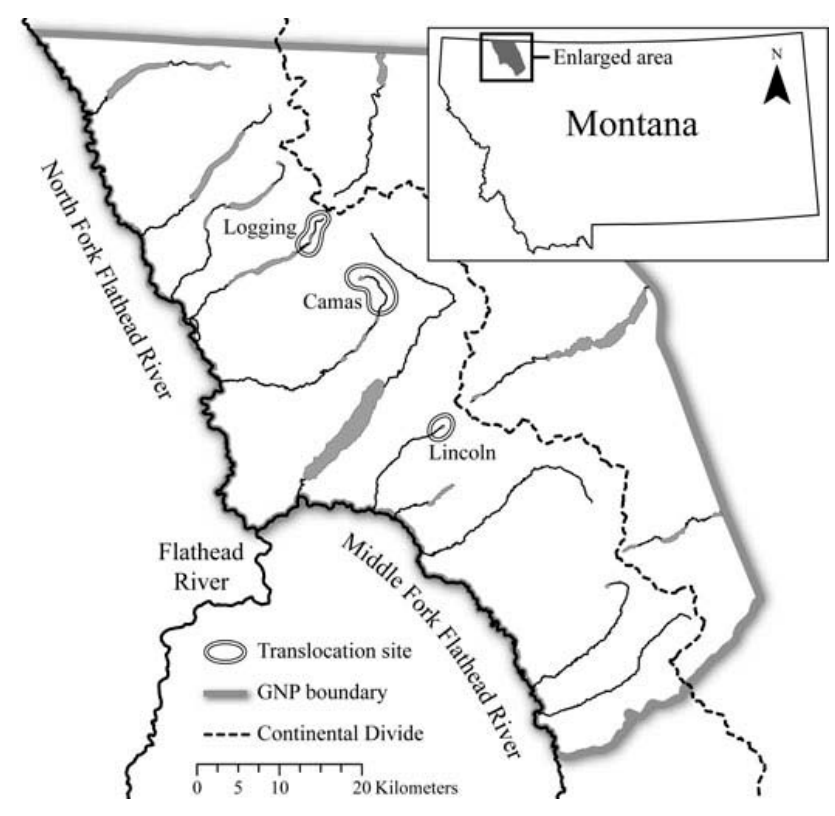

Figure C-2. Proposed sites of bull trout relocation on the west side of the Continental Divide in Glacier National Park, Montana. Relocation sites consisted of stream-and-lake networks within the Logging, Camas, and Lincoln Creek drainages. Figure from Galloway et al. (2016).

Managers in Glacier National Park are currently working to conserve the adaptive capacity of local bull trout stocks by capturing juvenile bull trout in the Logging Lake drainage and moving them upstream above an impassable waterfall to establish a population secure from invasive species and with sufficient genetic capacity to adapt to a changing climate over time. Chris Downs, fisheries biologist for Glacier National Park, provided the following account describing this effort. In 2014, managers captured and moved the last documented naturally produced year-class of bull trout (113 individuals) in the Logging Lake drainage. Due to the low abundance of wild juveniles remaining in the system and the limited diversity (genetic analysis suggested that they were represented by only 34 parents), managers subsequently initiated an effort to collect bull trout eggs from a "nearest neighbor" (Quartz Lake) and rear them in an isolation facility at Creston National Fish Hatchery for use in these efforts. They collected eggs in September 2016, and in June 2017 released approximately 750 age- 0 bull trout from 3 genetic families into tributary streams of Grace Lake, and an additional 200 age-1 bull trout in 2018. Conservation facility-reared juveniles are anticipated to be planted annually into Grace Lake through 2020. To date, managers in Glacier National Park are using genetics, marking of individual fish using OTC, fin clips, and PIT tags, recapture of fish using nonlethal trapnetting and electrofishing, and spawning surveys to document the success of the project. They have documented the annual survival of individuals from the wild fish transport group in 2014 and are continuing to monitor and evaluate the effort.

\section{Background}

Bull trout are members of the char subgroup of the family Salmonidae and are native to waters of northwestern North America. In the United States, bull trout range widely through the Columbia River and Snake River basins, extending east to headwater streams in Idaho and Montana (including the Saint Mary headwaters east of the continental divide), into Canada and southeast Alaska, and to 
the Puget Sound and Olympic Peninsula watersheds of western Washington and the Klamath River basin of south-central Oregon. Historically, bull trout also occurred in the Sacramento River basin in California (Hayes and Banish 2017).

Bull trout express both resident and migratory life history strategies. Resident forms of bull trout complete their entire life cycle in the tributary (or nearby) streams in which they spawn and rear. Migratory bull trout spawn in tributary streams, where juvenile fish rear for 1 to 4 years before migrating to either a lake (adfluvial form), river (fluvial form), or in certain coastal areas, to saltwater (anadromous). Resident and migratory forms may be found together, and either form may give rise to offspring exhibiting either resident or migratory behavior. The size and age of bull trout at maturity depends upon habitat capacity and subsequent life history strategy. Bull trout normally reach sexual maturity in 4 to 7 years; they frequently live for 10 years and occasionally for 20 years or more.

Of all native salmonids of the United States, bull trout have the most specific habitat requirements (Rieman and McIntyre 1995), which are often referred to as "the four Cs": Cold, Clean, Complex, and Connected habitat. These requirements include cold-water temperatures compared to other salmonids (often $<12^{\circ} \mathrm{C}$ ); the cleanest stream substrates; complex stream habitat including deep pools, overhanging banks and large woody debris; and connectivity between spawning and rearing areas and downstream habitats (Dunham and Rieman 1999).

Bull trout abundance and distribution declined significantly throughout the 20th century, leading to the species' listing in 1999 as 'threatened' under the US Endangered Species Act (ESA) of 1973. At the time of listing under the ESA, bull trout had been extirpated from an estimated $60 \%$ of their historical range, leaving populations localized and fragmented (USFWS 2015). Conservation actions have been implemented in many areas, and bull trout are currently considered generally stable rangewide, with some populations increasing, some decreasing, and some remaining static, but with essentially no known change in general distribution (USFWS 2015). The most recent short form status review completed in 2015 , as well as a more thorough review in 2008 , indicated that the threatened status is still appropriate (USFWS 2015).

Declines in bull trout numbers and distribution are due to threat factors that vary significantly based on location (USFWS 2015). Threats include increasing water temperatures, habitat loss and fragmentation, competition and hybridization with non-native fish species, and barriers to migration (Hayes and Banish 2017). Bull trout are extremely temperature-sensitive relative to other salmonids (Selong et al. 2001), and distributions during summer months are strongly tied to ambient stream temperatures (Dunham et al. 2003; Wenger et al. 2011). Bull trout exhibit complex life histories, and adults and subadults can seasonally demonstrate large upstream and downstream movements to access foraging and overwintering habitat (Swanberg 1997; Howell et al. 2010; Starcevich et al. 2012). Furthermore, strong relations exist between climate change, wildfires and stream temperature (Koontz et al. 2018), with critical implications for vulnerable bull trout populations (Isaak et al. 2010; Falke et al. 2015). Summer drought and a longer and more extensive fire season modifies riparian habitat deemed important for shading streams and maintaining low stream temperatures. In addition, post-fire floods and debris flows associated with high intensity fires can cause degradation of bull trout habitat, causing local extirpations of trout (Dunham et al. 2003). 
Climate change may also promote the spread and impact of nonnative trout on bull trout populations (Rieman et al. 2007; Ruesch et al. 2012; Eby et al. 2014). Brook trout (Salvelinus fontinalis) and lake trout (Salvelinus namaycush) have been identified as considerable threats to bull trout populations through predation (Martinez et al. 2009; Hansen et al. 2010; Fredenberg 2014), competition (Guy et al. 2011; Warnock and Rasmussen 2014), and nonintrogressive hybridization resulting in gametic wastage (Leary et al. 1993; Kanda et al. 2002; DeHaan et al. 2010). Recently, there has been growing concern regarding the effects of introduced brown trout (Salmo trutta) on bull trout populations in western Montana, USA, particularly in light of recent evidence suggesting expansion of brown trout into historical bull trout habitat in response to warming stream temperatures (Al-Chokhachy et al. 2016). In summary, although anthropogenic climate change was not considered a threat when bull trout were listed in 1999, it has become clear that direct and indirect effects of climate change on bull trout habitat may be substantial.

Efforts to recover bull trout throughout its range involve a combination of habitat restoration, population supplementation, suppression of nonnative species, relocation, and restoration of formerly extirpated populations. The USFWS' Recovery Plan for the Coterminous United States Population of Bull Trout establishes a strategic plan for recovery (USFWS 2015). The primary strategy for recovery of bull trout in the coterminous United States is to: (1) conserve bull trout so that they are geographically widespread across representative habitats and demographically stable in six recovery units; (2) effectively manage and ameliorate the primary threats in each of six recovery units such that bull trout are not likely to become endangered in the foreseeable future; (3) build upon the numerous and ongoing conservation actions implemented on behalf of bull trout since their listing in 1999, and improve our understanding of how various threat factors potentially affect the species; (4) use that information to work cooperatively with partners to design, fund, prioritize, and implement effective conservation actions in those areas that offer the greatest long-term benefit to sustain bull trout and where recovery can be achieved; and (5) apply adaptive management principles to implementing the bull trout recovery program to account for new information.

\section{Risk Assessment}

\section{$\underline{\text { Section I. Risk of No Managed Relocation Action }}$}

\section{I.A. Risk of No Action to the Target}

Bull trout will likely experience a range of exposures to climate change given their extensive geographic range (see above). Studies suggest that exposure will be greatest in the highest climate velocity parts of the stream network bull trout occupy, which is typically lower reaches in a stream network and lakes that may become increasingly stratified as temperatures increase (Isaak et al. 2015, 2016). In the Columbia River Basin, bull trout populations in low elevations and at their southern limit of their range were reported to be the most vulnerable (Wade et al. 2017). Isaak et al. (2010) estimated an 11-22\% loss of habitat length for bull trout from 1993 to 2006 in Boise River basin, Idaho resulting from the warming of summer stream temperatures, and Eby et al. (2014) showed that bull trout distributions in the East Fork Bitterroot River (Montana) have contracted as temperatures have increased over recent decades. Ruesch et al. (2012) evaluated future loss of the bull trout occupancy in the John Day River, Oregon, also in the southern part of their range, and 
predicted $80-100 \%$ loss of stream length and $93-100 \%$ loss of stream volume for bull trout according to a number of projected stream temperatures in 2100. Isaak et al. (2015) used large stream temperature and biological databases to show that thermal habitat in headwater mountain streams is highly resistant to temperature increases and that many populations of cold-water species (including bull trout) may be well buffered from climate change. This is in stark contrast to bull trout populations at low elevations and latitudes that are likely to be severely vulnerable to climate change related extirpation.

Natural and anthropogenic barriers to movement will play a large role in determining the sensitivity of bull trout populations and their adaptive capacity to track changes in climate (Rieman et al. 1997, 2007). Bull trout persistence into the future will depend on the lack of barriers to bull trout movement (e.g., dams, culverts) between currently occupied streams and those expected to remain thermally suitable (Isaak et al. 2015). Genetic variability in bull trout populations may also play a key role in reducing vulnerability to climate change, and thus is important when considering managed relocation (Mims et al. 2019). Kovach et al. (2015) reported that vulnerability related to climate change was negatively correlated with average genetic diversity in 24 watersheds in the Columbia River Basin. Those watersheds with lower average genetic diversity had the lowest habitat complexity, warmest stream temperatures, and the greatest frequency of winter flooding (Kovach et al. 2015). This study suggests that those places where genetic variability is depressed (a key component of adaptive capacity) are also the places where that variability is most needed to overcome habitat related climate vulnerability. This further highlights the vulnerabilities of these types of habitats.

The sensitivity of bull trout to changing water temperatures is heavily influenced by the interplay of climate change and non-native trout. It has been hypothesized that climate change may result in some species with lower temperature preferences, such as brook trout, retreating farther upstream to higher, cooler reaches as temperatures increase (Wenger et al., 2011) and local displacement or extirpations of bull trout due to increases in temperature and overlap with brook trout (Howell 2018). Similarly, Al-Chokhachy et al. (2016) showed the concurrent loss of bull trout and gain of nonnative brown trout distribution; however, it is unclear whether such changes are due to changes in climaterelated attributes (e.g., temperature) or exacerbated by interspecific interactions. In this study, expansion of brown trout appeared to be in part driven by regional air temperature and ensuing stream temperature warming during the 21 st century. Conversely, declines in bull trout appear to be at least partly thermally driven, as evidenced by multiple populations demonstrating significant declines in the absence of brown trout.

The vast majority of adfluvial bull trout populations in western Glacier National Park have dramatically declined in the last 25-30 years due to the invasion and establishment of nonnative lake trout (summarized in Galloway et al. 2016). Where they have been introduced, lake trout consistently displace native bull trout through predation and competition (Martinez et al. 2009). Prior to the recent invasion of nonnative lake trout, Glacier National Park contained one-third of the remaining natural lakes that supported threatened bull trout in the United States. West of the Continental Divide within Glacier National Park, lake trout have invaded 9 of the 12 lakes where bull trout are native 
(Fredenberg et al. 2007; D'Angelo and Muhlfeld 2013). In lakes for which data exist, bull trout populations have declined to the point of functional extirpation in less than 30 years post lake trout introduction (Fredenberg 2002; D’Angelo and Muhlfeld 2013; Downs et al. 2013).

There is moderate risk, with robust certainty and high agreement according to the published literature, of losing a significant number of bull trout populations within Glacier National Park.

\section{I.B. Risk of No Action to the Recipient Ecosystem}

The purpose of the managed relocation action is for the conservation of bull trout. Therefore, the risk of no action on the recipient ecosystem is not applicable.

Section II. Risks of Action Associated with the Target

II.A. Risk of No Action to the Relocated Individuals

Some data exists on recruitment success of bull trout associated with relocation events. For the Clackamas Bull Trout Reintroduction Program, Barry et al. (2014) report a 98\% survival rate from time of release to the start of spawning season, and also notes evidence of successful spawning.

There is low risk, with robust certainty and high agreement according to the published literature, of no survival or establishment of bull trout in the proposed relocation sites within Glacier National Park.

\section{II.B. Risk That the Target Source Population Cannot Withstand Diminished Numbers}

Relocations can result in risk to donor populations due to the number of individuals needed to create a viable population. Previous relocation assessments have recommended the use of bull trout donor populations with spawner abundances greater than 1,000 spawning adults/year to avoid risk to the donor population (Dunham et al. 2011). However, bull trout populations in Glacier National Park exist at much smaller geographic scales, and spawner abundances do not meet this criterion (Downs et al. 2013). Past research shows that self-sustaining populations with relative abundances generally greater than 0.2 fish/net-hour are often not threatened by competing or hybridizing species (Meeuwig and Guy 2007; Meeuwig et al. 2007). Galloway et al. (2016) reviewed previous studies to determine the relative abundance of bull trout populations downstream of the proposed sites and reported that the Camas site had relative abundances $>0.2$ fish/net-hour, whereas Lincoln and Logging Sites had relative abundances $<0.2$ fish/net-hour. Therefore, risk that the source population cannot withstand diminished numbers is both specific to the location and timing of the relocation event, and thus highly variable.

To evaluate genetic diversity, Galloway et al. (2016) created thresholds for distinguishing suitable donor populations based on heterozygosity estimates calculated for bull trout populations elsewhere in the Columbia River Basin (e.g., Kovach et al. 2015) and Glacier National Park (Meeuwig and Guy 2007; Meeuwig et al. 2010). Most of the connected populations of bull trout (i.e., populations not isolated by dispersal barriers) exhibit genetic heterozygosity estimates greater than or equal to 0.5 . Galloway et al. (2016) evaluated adaptive potential by referencing previous research that investigated the genetic heterozygosity of bull trout populations downstream of the proposed introduction sites (Meeuwig and Guy 2007, Meeuwig et al. 2010). The authors reported that observed genetic 
heterozygosity was $>0.50$ for Lincoln and Logging Sites, and $<0.50$ for Camas Site. Much like levels of relative abundance, population-level genetic variability was high, which translates into different levels of risk.

There is low to very high risk, with medium certainty and moderate agreement according to the published literature, that the source population cannot withstand diminished population size within Glacier National Park.

II.C. Risk That Removing the Target Will Negatively Impact a Key Function Role in the Source Ecosystem Assuming that a viable proportion of the original donor population remains after the relocation event, it is unlikely that any change in functional role in the donor ecosystem will occur.

There is low risk, with limited certainty and moderate agreement, that removing bull trout from the source populations will lead to a vacated functional role in the aquatic ecosystem. This is selfreported.

II.D. Risk of Causing Undesired Evolution in the Target

To the best of our knowledge, no published research has investigated whether a relocation event of bull trout will create some unwanted evolutionary trajectory in the relocated population. Risk is unknown.

Section III. Risks of Action to Non-targets in the Recipient Ecosystem

III.A. Risk of Target Transmitting Novel Disease or Associated Pest

Extensive disease screening procedures for fry, juveniles and adult bull trout have been established, including IHNV, IPNV, VHSV, OMV, ISAV, and M. cerebralis, as well as other treatable pathogens and parasites (Barry et al. 2014).

There is low risk, with robust certainty and high agreement according to the published literature, of bull trout introductions leading to the transmission of novel disease or associated pests.

III.B. Risk of Competitive Interaction Negatively Affecting the Distribution or Abundance of Non-targets

Bull trout will compete with other fish species in the recipient ecosystem. The local density of bull trout and the specific species present in the recipient ecosystem at the time of relocation will determine the magnitude of these competitive effects. Galloway et al. (2016) assessed the level and significance of this risk for three proposed sites in Glacier National Park and determined it to be low for Camas and Logging, and high for Lincoln, according to the presence of sensitive taxa collected during community-wide sampling, including boreal toads Bufo boreas boreas (Galloway 2014).

There is low to high risk, with robust certainty and high agreement according to the published literature, that competitive interactions by bull trout will affect the abundance or distribution of nontarget taxa in Glacier National Park. 
III.C. Risk of Consumptive Effects Reducing the Abundance or Distribution of Non-targets

Bull trout is an apex predator; therefore, it is likely that relocated individuals will predate upon invertebrates and vertebrates (fish, amphibians) in the recipient ecosystem. Relocation protocols recommend prohibiting introductions to locations where sensitive native species could be negatively impacted. Galloway et al. (2016) assessed the level and significant of this risk for three proposed sites in Glacier National Park and determined it to be low for Camas and Logging, and high for Lincoln, according to the presence of sensitive taxa collected during community-wide sampling (Galloway 2014).

There is low to high risk, with robust certainty and high agreement according to the published literature, that predatory interactions by bull trout will affect the abundance or distribution of nontarget taxa in Glacier National Park.

III.D. Risk of Driving Undesired Evolution in Non-targets

To the best of our knowledge, there is low risk, with low certainty and low agreement ${ }^{\mathbf{1 4}}$, of causing undesirable evolution in non-target species. This is self-reported.

Section IV. Risks of Action of Non-target, Higher Order Attributes of the Recipient Ecosystem

IV.A. Risk of Indirect and Negative Impacts on Ecosystem Structure

Bull trout is an apex predator; therefore it is likely that relocated individuals will predate upon and compete with invertebrates and vertebrates (fish, amphibians) in the recipient ecosystem. Depending on the local density of bull trout and the specific species present in the recipient ecosystem, the magnitude of food web effects may be small or large. If introduced to previously fishless lakes, reductions in larger copepods, cladocerans, and chaoborids are likely (Donald et al. 2001). Galloway et al. (2016) assessed the level and significance of this risk for three proposed sites in Glacier National Park and determined it to be low for Camas and Logging, and high for Lincoln, according to the presence of sensitive taxa collected during community-wide sampling (Galloway 2014).

There is low to high risk, with robust certainty and high agreement according to the published literature, that bull trout will cause negative impacts on food webs in Glacier National Park.

IV.B. Risk of Changing Ecosystem Function

To the best of our knowledge, there is low risk, with low certainty and low agreement, of changing ecosystem function. This is self-reported.

\footnotetext{
14 To reiterate, low agreement refers to a low level of agreement among data sources on the potential risk that is derived in this category.
} 
$\underline{\text { Section V. Risks Associated with Biological Invasion }}$

IV.A. Risk of Invasion Within the Intended Recipient Ecosystem

To the best of our knowledge, there is low risk, with low certainty and low agreement, of risk of invasion in the recipient locations in Glacier National Park. This is self-reported.

IV.B. Risk of Invasion Beyond the Recipient Ecosystem

Information on the complex migratory movements of fluvial, adfluvial, and anadromous life history forms has now increased our understanding of the extensive habitat use and connectivity requirements of the migratory life history form of bull trout (Muhlfeld and Marotz 2005; USFWS 2015). This information has affirmed that the use of migratory corridors is critical to the survival of bull trout. Moreover, metapopulation structure, genetic exchange, and recolonization of extirpated local populations are dependent on unrestricted movement of individuals (Dunham et al. 2011). Therefore, it is both expected and desired that bull trout move from the location of introduction. However, the risk of spread of relocated individuals from recipient location is likely to vary considerably. In the Clackamas River, no adults were found to disperse outside the relocation area over a 4-year period (Hudson et al. 2015). This information and results of other relocation events led Galloway et al. (2016) to report a low risk of spread of bull trout for three proposed sites in Glacier National Park.

There is low risk, with robust certainty and high agreement according to the published literature, that bull trout will invade beyond the introduction area in Glacier National Park.

\section{IV.C. Risk of Irreversibility of the Managed Relocation Action}

Evidence from previous attempts to physically remove non-native trout from lake and river ecosystems suggest that bull trout individuals can be removed should it become invasive. However, a vast literature documenting previous attempts to control and eradicate nonnative trout has shown that the success is very low and cost is very high; largely depending on distributional extent, local abundances, and the spatial and environmental context (e.g., Kulp and Moore 2000; Meyer et al. 2006; Peterson et al. 2008; Shepard et al. 2014).

There is moderate risk, with robust certainty and high agreement according to the published literature, that bull trout cannot be controlled should it become invasive.

IV.D. Other Risk

To the best of our knowledge and the information presented in Galloway et al. (2016), there are no other risks.

$\underline{\text { Section VI. Risks Associated with Socio-economic Values }}$

VI.A. Risk to a Culturally or Economically Important Species

In this case study, bull trout is likely to be the more societally valued species. Therefore, we are selfreporting that there is a low risk to other societally valued species, with moderate certainty and medium agreement. 


\section{VI.B. Risk to a Valued Ecosystem Service}

To the best of our knowledge, there is low risk, with low certainty and low agreement, of risk to a valued ecosystem service. This is self-reported.

\section{Conclusion}

Bull trout recovery throughout its range necessitates a combination of activities that include habitat restoration, population supplementation, suppression of nonnative species, relocation, and restoration of formerly extirpated populations (Figure C-3). Here, we completed a risk assessment of the managed relocation of bull trout making specific reference to relocation activities involving three populations into historically unoccupied habitats within their natal drainages in Glacier National Park, Montana. We found moderate risk of extinction of source populations due to rapid climateinduced warming of stream and lakes environments, and lower likelihood of fish movement to escape these changes because of natural and anthropogenic barriers (e.g., Hungry Horse Dam) to movement. In general, scientific evidence suggests relatively low risks of action associated with existing populations, although the ability of source populations to withstand diminished population size is uncertain. Risks of action associated with non-target impacts of bull trout relocation are highly variable as they relate to competitive or predator interactions with non-target organisms. Risks associated with bull trout relocation leading to invasive spread and impacts are generally low, although there is a moderate risk that bull trout populations cannot be controlled should they become invasive. Risks associated with socio-economic values were deemed low, but with moderate uncertainty.

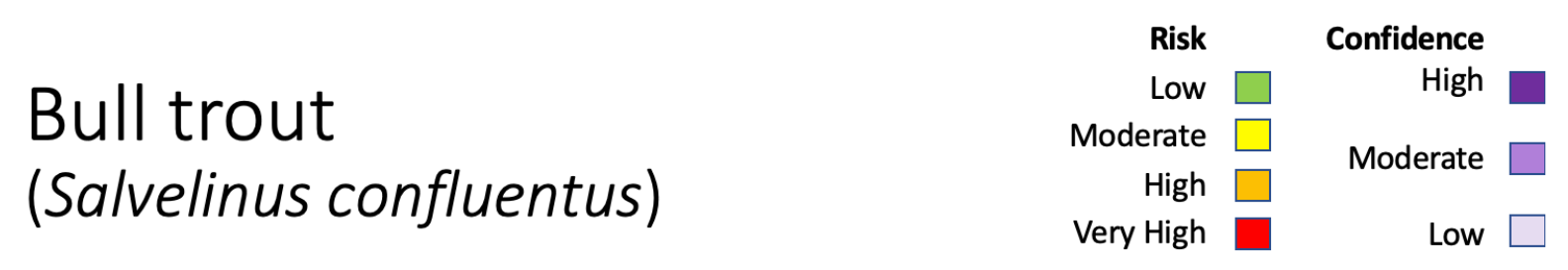

\begin{tabular}{|c|c|c|c|c|c|}
\hline Section & & & Risk Criteria & & \\
\hline Risk of: & A & B & $\mathrm{C}$ & D & $\mathrm{E}$ \\
\hline I. No action & $\begin{array}{l}\text { There is strong evidence of a } \\
\text { moderate risk of losing bull trout } \\
\text { populations within the park. }\end{array}$ & Not Applicable. & & & \\
\hline II. Action to the target & $\begin{array}{l}\text { Other introductions have } \\
\text { resulted in high survivorship. }\end{array}$ & \begin{tabular}{|l|} 
Low to very high risk that \\
remaining population could \\
withstand removal of individuals.
\end{tabular} & $\begin{array}{l}\text { Assuming a viable population } \\
\text { remains, it is unlikely to change } \\
\text { ecosystem function. }\end{array}$ & \begin{tabular}{|l|} 
No Information exists from which \\
to assess this risk.
\end{tabular} & No other risk assessed. \\
\hline III. Action to non-targets & $\begin{array}{l}\text { Extensive screening for disease } \\
\text { has a well-established } \\
\text { procedure. }\end{array}$ & $\begin{array}{l}\text { Low to high risk that bull trout } \\
\text { will impose competitive } \\
\text { interactions that impact other } \\
\text { species. }\end{array}$ & $\begin{array}{l}\text { Low to high risk that bull trout } \\
\text { will prey upon species and } \\
\text { impact other species } \\
\text { populations. }\end{array}$ & $\begin{array}{l}\text { There is very little evidence on } \\
\text { this aspect of risk. The expert } \\
\text { opinion of respondents is that } \\
\text { the risk is low. }\end{array}$ & No other risk assessed. \\
\hline $\begin{array}{l}\text { IV. Action to recipient } \\
\text { ecosystem }\end{array}$ & \begin{tabular}{|l|} 
As an apex predator, it is likely \\
that the bull trout will have \\
significant food web impacts if it \\
becomes abundant. The risk \\
varies from low to high.
\end{tabular} & $\begin{array}{l}\text { There is little evidence on this } \\
\text { aspect of risk. The expert opinion } \\
\text { of respondents is that the risk is } \\
\text { low. }\end{array}$ & No other risk assessed. & & \\
\hline V. Spread and invasion & \begin{tabular}{|l|} 
Based on expert opinion of \\
respondents.
\end{tabular} & $\begin{array}{l}\text { Low migratory potential of the } \\
\text { species. }\end{array}$ & $\begin{array}{l}\text { Previous evidence on removing } \\
\text { invasive fish suggests a moderate } \\
\text { risk. }\end{array}$ & No other risk assessed. & \\
\hline $\begin{array}{l}\text { VI. Adverse socio- } \\
\text { economic values }\end{array}$ & $\begin{array}{l}\begin{array}{l}\text { Bull trout is likely to be the more } \\
\text { socially valuable species than } \\
\text { things it may displace. Self- } \\
\text { reported. }\end{array} \\
\end{array}$ & $\begin{array}{l}\text { Respondents agree that this risk } \\
\text { is low. Self-reported. }\end{array}$ & No other risk assessed. & & \\
\hline
\end{tabular}

Figure C-3. Risk visualization table for the managed relocation of bull trout. 


\section{Literature Cited}

Al-Chokhachy, R., D. Schmetterling, C. Clancy, P. Saffel, R. Kovach, L. Nyce, B. Liermann, W. Fredenberg, and R. Pierce. 2016. Are brown trout replacing or displacing bull trout populations in a changing climate? Canadian Journal of Fisheries and Aquatic Sciences 73:1395-1404.

Barry, P. M., J. M. Hudson, J. D. Williamson, M. L. Koski, and S. P. Clements. 2014. Clackamas river bull trout reintroduction project, 2013 annual report. Oregon Department of Fish and Wildlife and U.S. Fish and Wildlife Service, $46 \mathrm{pp}$.

D’Angelo, V. S., and C. C. Muhlfeld. 2013. Factors influencing the distribution of native Bull Trout and Westslope Cutthroat Trout in streams of western Glacier National Park, Montana. Northwest Science 87:1-11.

DeHaan P. W., L. T. Schwabe, and W.R. Ardren. 2010. Spatial patterns of hybridization between bull, trout Salvelinus confluentus, and brook trout, Salvelinus fontinalis, in an Oregon stream network. Conservation Genetics 11:935-949.

Donald, D. B., R. D. Vinebrooke, R. S. Anderson, J. Syrgiannis, and M.D. Graham. 2001. Recovery of zooplankton assemblages in mountain lakes from the effects of introduced sport fish. Canadian Journal of Fisheries and Aquatic Sciences 58:1822-1830.

Downs, C.C., N. Muhn, and B. McKeon. 2013. Glacier National Park fisheries inventory and monitoring report, 2013. National Park Service, Glacier National Park, West Glacier, Montana.

Downs, C. C., C. R. Fredenberg, V. S. D’Angelo, C. C. Muhlfeld, and B. McKeon. 2015. Glacier National Park fisheries monitoring and management report, 2014. National Park Service. West Glacier, Montana.

Dunham, J. B., M. K. Young, R. E. Gresswell, and B.E. Rieman. 2003. Effects of fire on fish populations: landscape perspectives on persistence of native fishes and nonnative fish invasions. Forest Ecology and Management 178:183-196.

Dunham, J. B., K. Gallo, D. Shively, C. Allen, and B. Goehring. 2011. Assessing the feasibility of native fish reintroductions: a framework applied to threatened bull trout. North American Journal of Fisheries Management 31:106-115.

Dunham, J. B., and B. E. Rieman. 1999. Metapopulation structure of bull trout: influences of physical, biotic, and geometrical landscape characteristics. Ecological Applications 9:642-655.

Eby, L. A., O. Helmy, L. M. Holsinger, and M.K. Young. 2014. Evidence of climate-induced range contractions in bull trout Salvelinus confluentus in a Rocky Mountain watershed, U.S.A. PLoS ONE 9(6):e98812.

Falke, J. A., R. L. Flitcroft, J. B. Dunham, K. M. McNyset, P. F. Hessburg, and G. H. Reeves. 2015. Climate change and vulnerability of bull trout (Salvelinus confluentus) in a fire-prone landscape. Canadian Journal of Fisheries and Aquatic Sciences 72:304-318. 
Fredenberg, W. A. 2002. Further evidence that Lake Trout displace Bull Trout in mountain lakes. Intermountain Journal of Sciences 8:143-152.

Fredenberg, W. A., M. H. Meeuwig, and C. S. Guy. 2007. Action plan to conserve bull trout in Glacier National Park, Montana. U.S. Fish and Wildlife Service, Kalispell, Montana.

Fredenberg, C. 2014. Efficacy of suppressing non-native lake trout in an isolated backcountry lake in Glacier National Park. Master's thesis, Montana State University, Bozeman, Montana.

Galloway, B. T. 2014. Feasibility assessment for translocation of imperiled bull trout populations in Glacier National Park, Montana. Thesis, Montana State University, Bozeman, Montana.

Galloway, B. T., C. C. Muhlfeld, C. S. Guy, C.C. Downs, and W. A. Fredenberg. 2016. A framework for assessing the feasibility of native fish conservation translocations: applications to threatened bull trout. North American Journal of Fisheries Management 36:754-768.

Guy, C. S., T. E. McMahon, W. A. Fredenberg, C. J. Smith, D. W. Garfield, and B. S. Cox. 2011. Diet overlap of top-level predators in recent sympatry: bull trout and nonnative lake trout. Journal of Fish and Wildlife Management 2:183-189.

Glacier National Park (GNP), National Parks Conservation Association, USFWS, Flathead Valley Chapter of Trout Unlimited, University of Montana/National Park Service Rocky Mountain Cooperative Ecosystems Study Unit. 2003. The case for restoring bull trout in Glacier National Park ... and a framework to do it. National Park Service, West Glacier, Montana.

Hansen, M. J., D. Schill, J. Fredericks, and A. Dux. 2010. Salmonid predator-prey dynamics in Lake Pend Oreille, Idaho, U.S.A. Hydrobiologia, 650:85-100.

Hayes, M. F., and N.P. Banish. 2017. Translocation and reintroduction of native fishes: a review of bull trout Salvelinus confluentus with applications for future reintroductions. Endangered Species Research 34:191-209.

Howell, P. J., J. B. Dunham, and P. M. Sankovich. 2010. Relationships between water temperatures and upstream migration, cold water refuge use, and spawning of adult bull trout from the Lostine River, Oregon, U.S.A. Ecology of Freshwater Fishes 19:96-106.

Howell, P. J. 2018. Changes in native bull trout and non-native brook trout distributions in the upper Powder River basin after 20 years, relationships to water temperature and implications of climate change. Ecology of Freshwater Fish 27:710-719.

Hudson, J. M., R. Koch, J. Johnson, J. Harris, M. L. Koski, B. Galloway, and J. D. Williamson. 2015. Clackamas River bull trout reintroduction project, 2014 annual report. Oregon Department of Fish and Wildlife and U.S. Fish and Wildlife Service, 33 pp. 
Isaak, D. J., C. H. Luce, B. E. Rieman, D. E. Nagel, B. E. Peterson, D. L. Horan, S. Parkes, and G. L. Chandler. 2010. Effects of climate change and wildfire on stream temperatures and salmonid thermal habitat in a mountain river network. Ecological Applications 20:1350-1371.

Isaak, D. J., M. K. Young, D. Nagel, D. Horan, and M. C. Groce. 2015. The coldwater climate shield: Delineating refugia to preserve salmonid fishes through the 21 st century. Global Change Biology 21:2540-2533.

Isaak, D. J., M. K. Young, C. Luce, S. W. Hostetler, S. J. Wenger, E. E. Peterson, J. M. Ver Hoef, M. C. Groce, D. L. Horan, and D. E. Nagel. 2016. Slow climate velocities of mountain streams portend their role as refugia for cold-water biodiversity. Proceedings of the National Academy of Sciences, USA 113:4374-4379.

Kanda, N., R. F. Leary, and F. W. Allendorf. 2002. Evidence of introgressive hybridization between bull trout and brook trout. Transactions of the American Fisheries Society 131:772-782.

Koontz, E. D., E. A. Steel, and J. D. Olden. 2018. Stream thermal responses to wildfire in the Pacific Northwest. Freshwater Science 37:731-746.

Kovach, R. P., C. C. Muhlfeld, A. A. Wade, B. K. Hand, D. C. Whited, P. W. DeHaan, R. AlChokhachy, and G. Luikart. 2015. Genetic diversity is related to climatic variation and vulnerability in threatened bull trout. Global Change Biology 21:2510-2524.

Kulp, M. A., and S. E. Moore. 2000. Multiple electrofishing removals for eliminating rainbow trout in a small southern Appalachian stream. North American Journal of Fisheries Management 20:259-266.

Leary, R .F., F. W. Allendorf, and S. H. Forbes. 1993. Conservation genetics of bull trout in the Columbia and Klamath River drainages. Conservation Biology 7:856-685.

Martinez, P. J., P. E. Bigelow, M. A. Deleray, W. A. Fredenberg, B. S. Hansen, N. J. Horner, S. K. Lehr, R. W. Schneidervin, S. A. Tolentino, and A. E. Viola. 2009. Western lake trout woes. Fisheries 34:424-442.

Meeuwig, M. H., and C. S. Guy. 2007. Evaluation and action plan for protection of 15 threatened adfluvial populations of bull trout in Glacier National Park, Montana. U.S. Fish and Wildlife Service, Final Scientific Report, Kalispell, Montana.

Meeuwig, M. H., C. S. Guy, and W. A. Fredenberg. 2007. Research summary for action plan to conserve Bull Trout in Glacier National Park, Montana. U.S. Fish and Wildlife Service, Kalispell, Montana.

Meeuwig, M. H., C. S. Guy, S. T. Kalinowski, and W. A. Fredenberg. 2010. Landscape influences on genetic differentiation among bull trout populations in a stream-lake network. Molecular Ecology 19:3620-3633. 
Meyer, K. A., J. A. Lamansky, and D. J. Schill. 2006. Evaluation of an unsuccessful brook trout electrofishing removal project in a Small Rocky Mountain Stream. North American Journal of Fisheries Management 26:849-860.

Mims, M. C., C. Day, J. J. Burkhart, M. Fuller, J. Hinkle, A. Bearlin, J. Dunham, P. W. DeHaan, Z. A. Holden, and E. Landguth. 2019. Simulating demography, genetics, and spatially-explicit processes to inform reintroduction of a threatened char. Ecosphere 10:e02589.

Muhlfeld, C. C., and B. Marotz. 2005. Seasonal movement and habitat use by subadult bull trout in the upper Flathead River System, Montana. North American Journal of Fisheries Management 25:797-810.

Peterson, D. P., K. D. Fausch, J. Watmough, and R. A. Cunjak. 2008. When eradication is not an option: Modeling strategies for electrofishing suppression of nonnative brook trout to foster persistence of sympatric native cutthroat trout in small streams. North American Journal of Fisheries Management 28:1847-1867.

Rieman, B. E., D. Isaak, S. Adams, D. Horan, D. Nagel, C. Luce, and D. Myers. 2007. Anticipated climate warming effects on Bull Trout habitats and populations across the interior Columbia River Basin. Transactions of the American Fisheries Society 136:1552-1565.

Rieman, B. E., D. C. Lee, and R. F. Thurow. 1997. Distribution, status, and likely future trends of Bull Trout within the Columbia River and Klamath basins. North American Journal of Fisheries Management 17:1111-1125.

Rieman, B. E., and J. D. McIntyre. 1993. Demographic and habitat requirements for conservation of bull trout. General Technical Report INT-302. U.S. Forest Service, Intermountain Research Station, Boise, Idaho.

Rieman, B.E., and J. D. McIntyre. 1995. Occurrence of Bull Trout in naturally fragmented habitat patches of varied size. Transactions of the American Fisheries Society 124:285-296.

Ruesch, A. S., C. E. Torgersen, J. J. Lawler, J. D. Olden, E. E. Peterson, C. J. Volk, and D. J. Lawrence. 2012. Projected climate-induced habitat loss for salmonids based on a network model of stream temperature. Conservation Biology 5:873-882.

Selong, J. H., V. McMahon, A. V. Zale, and F. T. Barrows. 2001. Effect of temperature on growth and survival of Bull Trout, with application of an improved method for determining thermal tolerance in fishes. Transactions of the American Fisheries Society 130:1026-1037.

Shepard, B. B., L. M. Nelson, M. L. Taper, and V. Z. Alexander. 2014. Factors influencing successful eradication of non-native brook trout from four small Rocky Mountain streams using electrofishing. North American Journal of Fisheries Management 34:988-997. 
Starcevich, S. J., P. J. Howell, S. E. Jacobs, and P. M. Sankovich. 2012. Seasonal movement and distribution of fluvial adult bull trout in selected watersheds in the mid-Columbia River and Snake River basins. Plos ONE 7(5):e37257.

Swanberg, T. R. 1997. Movements of and habitat use by fluvial bull trout in the Blackfoot River, Montana. Transactions of the American Fisheries Society 126:735-746.

U.S. Fish and Wildlife Service (USFWS). 1999. Endangered and threatened wildlife and plants; determination of threatened status for bull trout in the conterminous United States. Federal Register 64:58910-58933.

U.S. Fish and Wildlife Service (USFWS). 2005. Endangered and threatened wildlife and plants; designation of critical habitat for the bull trout. Federal Register 70:56212-56311.

U.S. Fish and Wildlife Service (USFWS). 2015. Recovery plan for the coterminous United States population of bull trout (Salvelinus confluentus). U.S. Fish and Wildlife Service, Portland, OR

Wade, A. A., B. K. Hand, R. P. Kovach, G. Luikart, D. C. Whited, and C. C. Muhlfeld. 2017. Accounting for adaptive capacity and uncertainty in assessments of species' climate-change vulnerability. Conservation Biology 31:136-149.

Warnock, W. G., and J. B. Rasmussen. 2014. Comparing competitive ability and associated metabolic traits between a resident and migratory population of bull trout against a non-native species. Environmental Biology of Fishes 97:415-423.

Wenger, S. J., D. J. Isaak, C. H. Luce, H. M. Neville, K. D. Fausch, J. B. Dunham, D. C. Dauwalter, M. K. Young, M. M. Elsner, B. E. Rieman, A. F. Hamlet, and J.E. Williams. 2011. Flow regime, temperature, and biotic interactions drive differential declines of trout species under climate change. Proceedings of the National Academy of Sciences, USA 108:14175-14180. 


\section{Case Study: Risk Assessment of the Managed Relocation of the Karner Blue butterfly (Plebejus melissa samuelis)}

Sarah Skikne and Jessica J. Hellmann (University of Minnesota)

\section{Hypothetical Action}

We completed a risk assessment of managed relocation of the Karner Blue Butterfly (Plebejus melissa samuelis, KBB). For this case study, we assessed a hypothetical set of actions consisting of the following steps: (1) collect KBBs from populations in two nearby source sites - Otto Township and White River - in Huron-Manistee National Forest in west-central lower-peninsula Michigan, (2) propagate KBBs from these collected individuals in captivity for approximately one year, and (3) release captive-bred KBBs at Sleeping Bear Dunes National Lakeshore, MI (SLBE), approximately $150 \mathrm{~km}$ north of the source sites (Figure C-4). These steps are assessed on the assumption that managed relocation is a useful and desirable strategy for preserving the KBB into the future, that the source population for the managed relocation is healthy, and that the recipient site is ecologically and climatically suitable in the near-term.

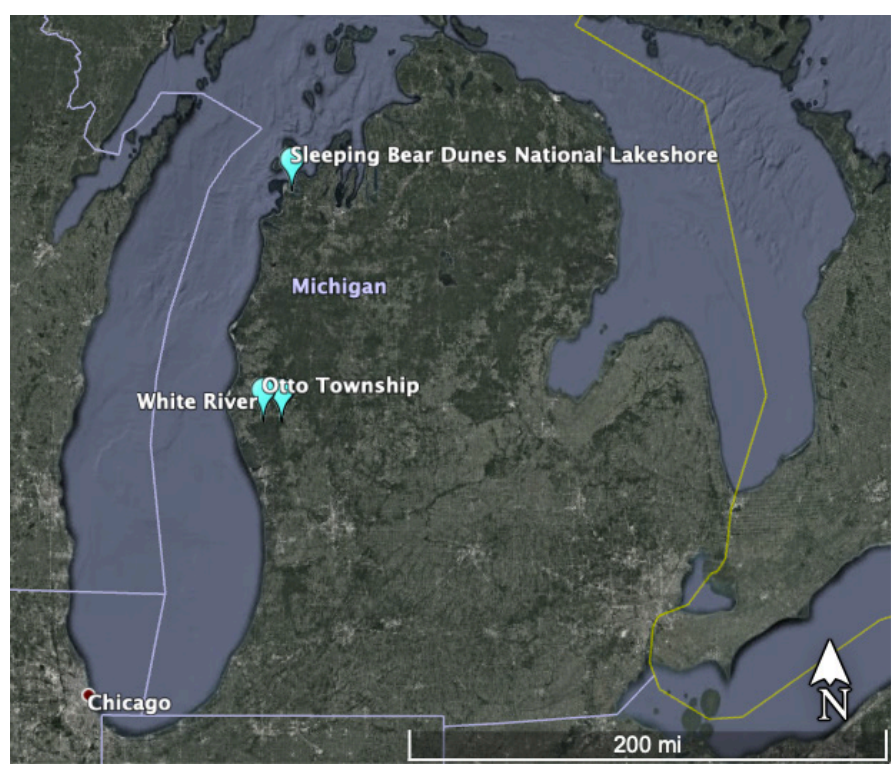

Figure C-4. Map of Michigan's lower peninsula showing source sites (Otto Township and White River, both in Huron-Manistee National Forest) and recipient site (Sleeping Bear Dunes National Lakeshore) assessed in risk assessment of hypothetical managed relocation of Karner Blue Butterfly Plebejus melissa samuelis.

\section{Background}

The KBB is a small, bivoltine butterfly (Figure C-5) that relies on a single host plant, lupine (Lupinus perennis), as well as nectar plants. The subspecies was historically found in savanna/barrens ecosystems across 12 states from Minnesota to Maine, as well as Ontario, Canada. KBB distributions and numbers have been reduced via development, fragmentation, and succession of savanna/barrens ecosystems, leading to federal listing in 1992 (USFWS 2003). We chose the KBB because the subspecies provides an example managed relocation for an invertebrate, is federally listed and wellstudied, and is considered vulnerable to climate change (described in section I below). 


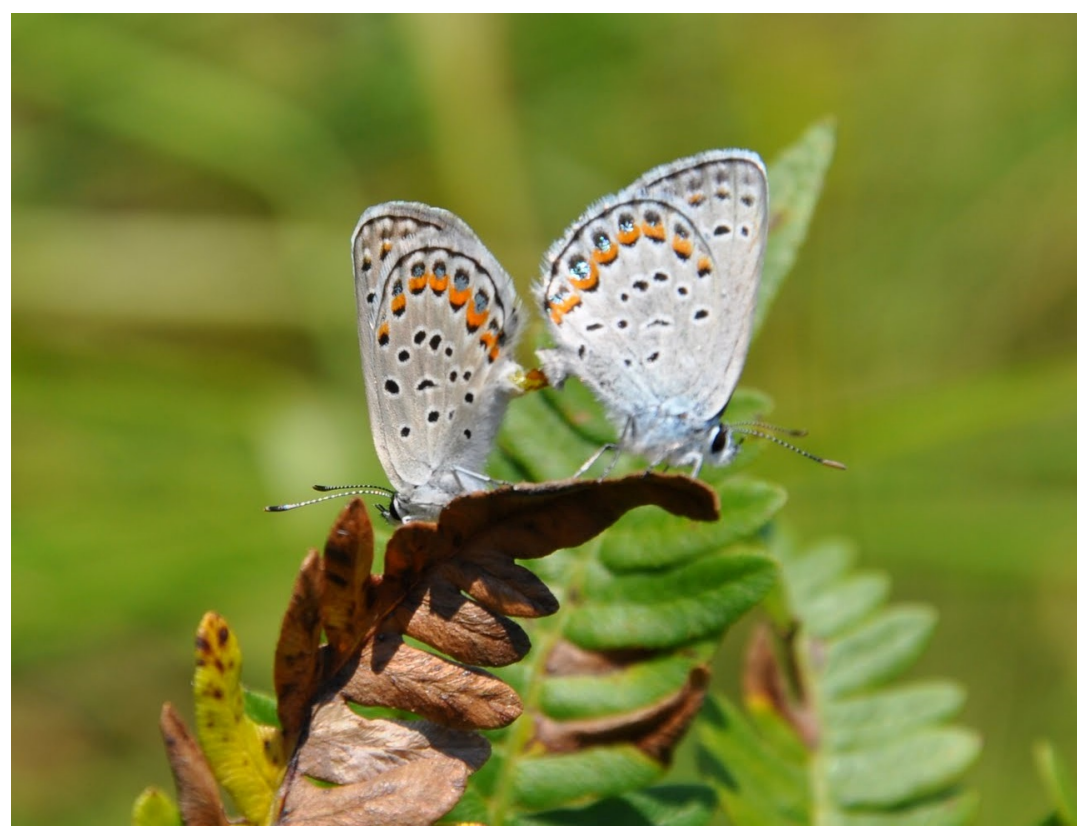

Figure C-5. Karner blue butterflies (Plebejus Melissa samuelis) mating. Photo credit: G. Wu.

We chose the source and recipient sites for the case study because they are generally aligned northto-south (i.e., in the general direction of moving climate envelopes, Fig. 1), and the source sites have robust populations (C. Hoving, pers. comm.). Moreover, the recipient site includes areas of sandy soil and is proximate to the lake/dunes, which is comparable to (some) KBB habit associated with drier soils and oak savanna, e.g., recently extirpated population at Indiana Dunes National Park. We have not conducted a formal assessment of recipient site suitability because this is a hypothetical case study, but an actual managed relocation assessment process would need further, more detailed analysis of the recipient area (here SLBE) and potential recipient subsites (the specific location within SLBE where the actual release would occur). Such assessments would include macro- and micro-climate suitability over varying time periods and their match with the source site climate, habitat availability, and communities of interacting species (e.g., nectar species, ants).

Although SLBE is within the larger range of lupine, lupine is not known in SLBE or the counties which encompass it (Reznicek et al. 2011). Lupine would therefore need to be introduced prior to managed relocation of the KBB. Here we assume there are sufficient areas with appropriate microclimates and substrates for such an endeavor, and that lupine would be planted and actively managed within pre-defined boundaries. In the following, we do not consider lupine to be a "target species" in sections I and II, since it is not being moved for the sake of its conservation, but we do consider it a "target species" in sections III, IV and V given the potential risks associated with its introduction to SLBE. Questions about where to source lupine for planting at SLBE would need to be addressed with additional studies and risk assessment. For example, experiments might include how KBB perform on lupine from neighboring sites vs. KBB source sites, as well as how lupine from varying sources perform when planted at SLBE. 
Finally, we note that such a managed relocation of the KBB might be the first of a larger sequence of managed relocations needed to keep pace with climate change. For example, a population established at SLBE might become a source population for a second managed relocation to a more northern site, such as Pictured Rocks National Lakeshore.

\section{Risk Assessment}

\section{Section I. Risks of No Managed Relocation Action}

\section{I.A. Risk of No Action to the Target}

The extinction risk for this particular pair of source populations has not been studied, but is plausible since the KBB is considered "highly vulnerable" to climate change in Michigan (Hoving et al., 2013) and will increase over time as the subspecies' northward-moving climate envelope ultimately leaves Otto Township and White River behind (Hällfors et al. 2016). Below, we describe the adaptive capacity, sensitivity, exposure and overall vulnerability to climate change for the subspecies as a whole, and where available include information on the specific Michigan populations of interest.

The KBB's adaptive capacity - its ability to tolerate change through natural range shifts, altered behavior, or evolution - is likely to be low. Unaided range shifts are unlikely, since KBB habitat is highly fragmented (US Fish and Wildlife Service, 2003) and individuals disperse only short distances (Knutson and Kwilosz, 1999). This is illustrated by preliminary data suggesting low gene flow between nearby ( $\sim 1 \mathrm{~km}$ apart) populations with only minor barriers to movement at Indiana Dunes National Park (Grundel, 2013). Adaptation "in place" via behavioral changes is also unlikely given the subspecies' reliance on a single host plant species. Finally, isolation and small population sizes may limit the standing genetic variation needed for adaptive evolution in the face of changing conditions; for example, preliminary data from Indiana Dunes National Park showed low withinpopulation diversity for isolated small populations (Grundel, 2013).

Lab studies show the subspecies' considerable sensitivity to warming conditions at several life stages (Bristow, 2017). Moreover, Dirig (1994) anticipated sensitivity to climate change due to the alignment of KBB's southern distribution limit with the snow pack line, although there are other potential explanations for this distribution. The source populations in Michigan have historically experienced low temperature seasonality due to the lake's buffering effects (Schuurman \& Hoving et al., unpublished report), and so may be more sensitive to climatic extremes because they have not faced them before. Finally, the distribution of KBB's "eastern" population (which includes the source populations) is most explained by mean temperature of the warmest quarter (Hällfors et al., 2016), further suggesting sensitivity to temperature changes.

The KBB may also be sensitive to indirect climate impacts. Abiotic impacts on other butterfly species have been shown to be mediated by host plants (e.g., Hellmann, 2002). For the KBB, indirect impacts could include changes in lupine and the nectar plant and ant communities on which the subspecies depends. Lupine and nectar plants may be vulnerable to climate-driven changes in nonnative plant species, soil moisture, and disease, all with potential downstream impacts on the KBB. Even though lupine is adapted to dry conditions associated with sandy soils, it has been shown to be sensitive to changes in precipitation (Schneider et al., 2014) and the juvenile stage is sensitive 
to drought conditions (Halpern, 2003). On the other hand, lupine may also benefit from increasing $\mathrm{CO}_{2}$ concentrations (Lee et al., 2003).

KBB populations in Michigan experienced up to $\sim 1-^{\circ} \mathrm{C}$ increase in mean annual temperature and $\sim 150$-mm increase in mean annual precipitation between the 40-year normal (1960-2000) and 20042013 (Schuurman \& Hoving et al., unpublished report). In the future, such changes are expected to intensify. In the future, the source populations are projected to experience relatively low levels of warming and precipitation changes compared to other parts of the KBB range, but relatively large decreases in snowfall and days with snow pack, and relatively large shifts in the date of the last spring freeze (Schuurman \& Hoving et al., unpublished report). Moreover, climate envelope modeling of the KBB eastern population (which includes the source sites) shows low overlap between the subspecies' climatic niche and its current distribution by 2030s, and almost no overlap by the 2070s (Hällfors et al., 2016).

The combination of low adaptive capacity and high sensitivity have apparently led to local extinctions of KBBs in sites with high climate exposure. Early spring warming and severe drought apparently led to the final extinction of a declining population at the (formerly) warmest and southernmost KBB site at Indiana Dunes National Park (Patterson et al., in review). This was despite extensive habitat and microclimate management (Patterson et al., in review; R. Grundel, pers. comm.) and a large population at the time of listing (US Fish and Wildlife Service, 2003). Such climate change-driven extinction of isolated populations have been documented in other butterfly species (McLaughlin et al., 2002).

Given the above robust evidence with high agreement ${ }^{15}$, primarily from published data on the target $^{16}$, we have high confidence that the risk of extinction of the source population is medium.

\section{Section II. Risks of Action Associated with the Target}

\section{II.A. Risk of Action to the Relocated Individuals}

Here, we first address the risk during collection and captive-rearing, and second the risk post-release. Well-documented and -developed methods exist for collecting and propagating KBBs, individual survival rates in captivity are relatively high, and efforts to breed captive colonies have been shown to succeed (Webb, 2010). Population restorations of historically occupied sites have occurred in three states, and population augmentations have occurred in two (Webb, 2010). Moreover, many KBB restoration attempts have been successful (U.S. Fish and Wildlife Service, 2012), contributing significantly to KBB recovery within its historical range. The individuals subject to a managed relocation would not face any different risks than these previous actions up until the time of release.

\footnotetext{
${ }^{15}$ Here and below, we deemed the level of agreement as "high" if all described evidence supported the same conclusion, and "medium" if there was any contrasting evidence or speculation about contrasting outcomes. There were no areas of risk which we deemed to have "low" agreement.

${ }^{16}$ We relied on numerous kinds of available sources, and some evidence carries more weight than others. However, here and in the following, we opted to list the source type that was used the most often. Where source types were used in equal measure, we list the source type as "other".
} 
The primary issue is whether individuals will survive and establish a self-sustaining population postrelease. Given that KBBs have been successfully restored at empty sites within the KBB's historical range (Webb, 2010), the specific question is how a beyond-historical-range recipient site might differ from a within-range site, and if this might cause additional risk for KBBs. For this case study, we assume that SLBE and recipient subsites within have suitable microclimates, shade heterogeneity, nectar species, etc. for KBBs. We also assume that native lupine can and will be established prior to release in appropriate subsites within SLBE, which is plausible given that lupine has been successfully propagated (Webb, 2010) and established in advance of KBB restorations in the past (R. Grundel, pers. comm.). We discuss the risk of inbreeding depression limiting establishment below in section II.D.

One subcomponent of post-release risk would arise if KBB populations are locally adapted to the source sites and not sufficiently adapted to the recipient site to survive and establish. Successful KBB restorations have had longer geographic distances between source and recipient site than is proposed here, suggesting that such relocation distances are not risky per se (this case would constitute a relocation of $\sim 150 \mathrm{~km}$; KBBs restored in Michigan and Ohio were sourced from $\sim 200 \mathrm{~km}$ away, in Allegan State Game Area, Michigan, and restorations in Concord, New Hampshire were sourced from $\sim 190 \mathrm{~km}$ away, from Albany, New York). Moreover, the source sites are near Lake Michigan (Fig. 1) and therefore likely to possess other habitat characteristics in common with SLBE. However, while differentiation in KBB climatic niches has been studied at much larger scales (Hällfors et al., 2016), it is unknown how locally adapted KBB populations are to climate, nectar plants, ant communities, etc., nor whether such adaptation might limit KBB survival and establishment at the recipient site. There is some evidence that local adaptation to lupine is not a limitation to relocation, as evidenced by the observation that larvae from out-of-state capture sites successfully grew and metamorphosed on native lupine in restored $\mathrm{OH}$ and $\mathrm{NH}$ populations (US Fish and Wildlife Service, 2003). In other butterflies, however, local adaptation to host plant species or population has been observed (e.g., Pelini et al., 2010). This phenomenon could affect the fitness of introduced individuals, even if plant preferences are not pronounced.

Although the evidence for pre-release risks is robust with high agreement, the evidence for postrelease risk is only medium-strength with medium agreement; we use those latter levels for this area of risk overall. Therefore, we rate the above as medium-strength evidence with medium agreement, primarily from published data on the target, and therefore have medium confidence that risk in this area is medium.

\section{II.B. Risk That the Target Source Population Cannot Withstand Diminished Numbers}

The source sites were chosen because they have relatively robust populations (C. Hoving, pers. comm.), so we do not anticipate that removing KBBs will directly threaten the source population. Moreover, the risk to the source population would be the same as is normally undertaken for restorations and augmentations. In addition, taking from two populations limits the impact on any single population, and captive propagation limits the number of individuals that need to be removed from the source populations in the first place. In general, individuals can generate a large number of offspring within the first few generations of collection; for example, $\sim 15$ individuals were captured to 
establish a captive population that produced $\sim 1,000$ adults over three years (R. Grundel, pers. comm.). However, the risk of adverse impacts on the source population cannot be ruled out and must be assessed. For this case study, we assume that managers would monitor the source populations before and after removal of KBBs, in order to evaluate the impact of removal so that plans can be modified should adverse impacts occur (US Fish and Wildlife Service, 2003). We also assume that adaptive management and monitoring practices would ensure that continued removals are justified (or not) based on the success (or failure) of the collected/propagated/released populations.

Given the above medium-strength evidence with medium agreement, primarily from other sources (in this case a mixture of experts and self-reporting), we have medium confidence that risk in this area is low.

II.C. Risk That Removing Individuals of the Target Will Negatively Impact a Key Function in the Source Ecosystem

This relocation would not remove all KBBs from the source site populations and would seek to avoid any significant impact to the source populations. This effort would further limit the number of KBBs removed from source populations by using captive propagation. Moreover, the KBB plays no known unique roles in ecosystems in which they occur.

We rate the above as medium-strength evidence with high agreement, from self-reporting, and therefore have medium confidence that risk in this area is low.

\section{II.D. Risk of Causing Undesired Evolution in the Target}

Captive butterfly populations can experience inbreeding, morphological changes, and other problems (summarized in Crone et al., 2007), and captive KBBs can have low oviposition rates once released (Pickens and Root, 2007). However, captive propagation is necessary to minimize numbers taken from the wild (see above) and negative impacts can be mitigated by limiting time in captivity (in this case study we suggest limiting to one year) and following best practices outlined in Webb (2010), e.g., collecting from more than one site to increase genetic diversity. The risk of problems induced by captivity would be the same risk as is taken for restoration and augmentation programs using captive populations and may negatively impact how well the released population can sustain itself but would not negatively impact any other factors at the recipient site.

Once released, we do not know how likely it is that the population would become genetically distinct from the source populations, be subject to substantially distinct selective forces, or result in an unwanted evolutionary trajectory. We do not think the risk would be greater than for any other relocation to a site chosen for its suitability for the taxon but nevertheless beyond its historical range.

The evidence for problems with captivity is medium-strength, while the evidence for problems postrelease is limited; we use those latter level for this area of risk overall. We therefore rate the above as limited-strength evidence with medium agreement, from primarily self-reporting, and have low confidence that risk in this area is medium. 
II.E. Other

None.

Section III. Risks of Action to Non-targets in the Recipient Ecosystem

III.A. Risk of Target Transmitting Novel Disease or Associated Pest

The main recognized concern in this area of risk is Wolbachia, an endosymbiotic bacteria that could potentially be spread via KBB relocations; however Wolbachia has not been detected in KBB populations east of Lake Michigan (Nice et al., 2009). KBBs do not have any other known pests or diseases in the wild. In addition, best practices will be used to prevent, monitor for, and contain any outbreaks during captive KBB propagation (Webb 2010), further decreasing this area of risk. Finally, the closely related Melissa blue Plebejus melissa melissa is not known in Michigan (NatureServe, 2019); the lack of closely related species at the recipient site decreases the chances that local species would be susceptible to any KBB-transmitted pests or diseases.

We assume best practices would be followed to minimize the risk of transmitting lupine-associated pests or diseases to other plant species at SLBE. Because the site is within the broader lupine distribution and lupine exists in adjacent counties (Reznicek et al., 2011), we do not think this poses a large risk.

The above is robust evidence for KBB-associated pests and diseases and medium-strength evidence for lupine-associated pests and diseases; we use the latter for this overall area of risk. We therefore deem the above as medium-strength evidence with high agreement, primarily based on published data on the target, and have medium confidence that risk in this area is low.

III.B. Risk of Competitive Interaction Negatively Affecting the Distribution or Abundance of Non-targets

KBBs are nectar plant generalists and therefore unlikely to monopolize any one species at the recipient site. Nevertheless, it is plausible that they would compete for nectar plants or tending ants with other insects.

Introducing lupine will create competition for space and possibly water with other plant species. The extent to which it will cause a shift in the plant community will depend on lupine's density and ability to spread; Fuller (2008) suggests that tens of thousands of lupine are required to create a viable KBB population, so we anticipate that this this risk is high. Further study would be needed to determine the necessary number and size of planting areas; if the total area affected is high relative to available habitat at SLBE, this would further support high risk in this area.

The plant community at SLBE includes the federally-threatened dune thistle Cirsium pitcher. Lupine and dune thistle tend to occupy different microtopographic and microclimatic niches (R. Grundel, pers. comm.), and potential interactions could be further mitigated by ensuring that lupine are planted in areas without dune thistle. Additional studies would be needed and considered when choosing subsites for planting within SLBE.

We deem the above as medium-strength evidence with medium agreement, primarily based on self-reporting, and have medium confidence that risk in this area is high. 
III.C. Risk of Consumptive Effects Reducing the Abundance or Distribution of Non-targets

KBB's sole host plant would be added for the purpose of the managed relocation (i.e., nothing else relies on it currently at the recipient site).

We deem this as robust evidence with high agreement, primarily based on self-reporting, and therefore have high confidence that risk level in this area is low.

III.D. Risk of Driving Undesired Evolution in Non-targets

Managed relocation of KBBs is unlikely to create strong selective forces on nectar sources or ant communities, since it is a generalist in both regards. While the KBB could theoretically affect the relative success of one ant or nectar species relative to another (by changing its abundance, competitors, etc.), such an impact is purely speculative. There are no taxa within the recipient ecosystem with which KBBs would be capable of breeding or hybridizing (Plebejus melissa melissa is not known in Michigan, NatureServe, 2019).

It is not known how introduction of lupine might impact the evolution of other species at the recipient site (e.g., via competition, as above, or via changes to nitrogen availability or disturbance described below).

We deem the above as limited-strength evidence with medium agreement, primarily based on self-reporting, and therefore have low confidence that risk level in this area is medium.

Section IV. Risks of Action to Non-target, Higher Order Attributes of the Recipient Ecosystem

IV.A. Risk of Indirect and Negative Impacts on Ecosystem Structure

The KBB is unlikely to create indirect impacts on the food web. The KBB's sole host plant would be added for the purpose of the managed relocation (i.e., nothing else relies on it currently at the recipient site). KBBs are nectar plant generalists, and therefore unlikely to monopolize any one species and create significant indirect impacts on competitors for nectar. They are unlikely to cause changes in predator species because they would comprise a relatively small biomass in the system; however, historically KBBs demonstrated the capacity to become dense and abundant under the right conditions (R. Grundel, pers. comm.), so this risk cannot be ruled out.

Lupine is probably more likely to impact the food web, especially given the high number of lupine needed to create a viable KBB population (Fuller, 2008). Lupine could provide a new food source for an herbivore and thereby reduce herbivory on a different plant species, with cascading and difficultto-predict impacts on the food web.

We deem the above as medium strength evidence with medium agreement, primarily based on self-reporting, and therefore have medium confidence that risk in this area is medium.

\section{IV.B. Risk of Changing Ecosystem Function}

There is very low risk that the KBB itself could alter ecosystem functions at SLBE. However, introducing lupine could change ecosystem function. Fuller (2008) suggest a viable KBB population requires tens or hundreds of thousands of plants; establishing any plant in such numbers could alter 
ecosystem function, for example via soil / sand hydrology. In addition, lupine fixes nitrogen, and maintaining lupine may require adding ongoing disturbance such as fire (Kwilosz and Knutson, 1999; Pickens and Root, 2009) to SLBE. These factors could alter ecosystem function at the recipient site, and such changes may have ripple effects on other species. For example, nitrogen fixation by other, native lupine species ${ }^{17}$ can facilitate invasions of weedy species into sandy habitats (e.g., Maron and Connors, 1996). As above, additional studies would be needed to fully assess this risk and the potential for mitigation.

We deem the above as medium strength evidence with high agreement, primarily based on other sources (in this case a mixture of published data on the target and self-reporting), and therefore have medium confidence that risk in this area is high.

IV.C. Other

None.

$\underline{\text { Section V. Risks Associated with Biological Invasion }}$

V.A. Risk of Invasion Within the Intended Recipient Ecosystem

The KBB does not have traits associated with invasion (e.g., it is a host plant specialist with narrow environmental tolerances), there are no examples of such outcomes from prior KBB relocations, and there are relatively few pest butterfly species overall. However, historically KBBs have demonstrated the capacity to become dense and abundant under the right conditions (R. Grundel, pers. comm.), so this risk cannot be ruled out.

There are other species of lupine that are invasive (e.g., Pickart et al., 1998; Valtonen et al., 2006). We think invasion of lupine into pitcher's thistle areas is unlikely given their differences in preferred microclimates (as above). SLBE is within the lupine native range but currently unoccupied by lupine (lupine is in adjacent counties, but not the counties encompassing SLBE, Reznicek et al., 2011). Determining what limits lupine in SLBE counties would help inform the risk of invasion within SLBE (as well as how difficult it will be to establish there). For example, if studies showed that lupine is limited by substrate, then we assume this limitation could be overcome for the sake establishment, but that the likelihood of invasion beyond planted areas might be low.

We rate the above as medium-strength evidence with medium agreement, from primarily selfreporting, and therefore have medium confidence that the risk in this area is medium.

\section{V.B. Risk of Invasion Beyond the Recipient Ecosystem}

Captively-bred, restored KBB populations have colonized nearby ( $\sim 1 \mathrm{~km}$ away), previously unoccupied areas in Indiana and Ohio (U.S. Fish and Wildlife Service, 2012). However, such a

\footnotetext{
${ }^{17}$ Karner blue butterflies use a single native lupine species as nectar sources and egg-laying sites. However, there are other native species in the same genus that may have particular ecosystem impacts on the native host plant. These species are collectively referred to, throughout, as 'other native lupines.'
} 
colonization to nearby sites around SLBE would be unlikely because it would require lupine spreading first, and in any case may not be seen as a negative outcome.

The counties encompassing SLBE are bordered by Lake Michigan on one side and counties with lupine on the other, so spread of lupine beyond SLBE and further into the SLBE counties would not necessarily constitute a problem. As above, we note that there are other invasive species of lupine, and that understanding of the limitations on lupine in SLBE counties would inform the risk of invasion beyond SLBE.

We rate the above as medium-strength evidence with medium agreement, from primarily selfreporting, and therefore have medium confidence that risk in this area is medium.

\section{V.C. Risk of Irreversibility of the Managed Relocation Action}

Active management would facilitate keeping lupine from spreading beyond pre-defined boundaries. In addition, assuming for this case study that additional disturbance at SLBE is required to maintain lupine, then removal of disturbance would allow for control of lupine and subsequently KBB populations. For example, if fire is used to maintain lupine habitat, fire cessation could be used to cause lupine (and thus the KBB) to decline.

We rate the above as medium-strength evidence with high agreement, from primarily selfreporting, and therefore have medium confidence that the risk in this area is low.

V.D. Other

The risk that lupine could promote other plant invasions via nitrogen fixation is captured above in section III.B.2 ("Risk of changing ecosystem function").

Section VI. Risks Associated with Socio-economic Values

\section{VI.A. Risk to a Culturally or Economically Important Species}

The economically and culturally important species at SLBE and nearby natural areas are the plants and animals that attract wildlife viewing and tourism, as well as species of conservation concern (e.g., Piping plover Charadrius melodus, dune's thistle). For this case study, we assume that should lupine spread beyond park boundaries, risks to other species (e.g., crop species) are low. Therefore, the risk here is a synthesis of those risks described in sections III and IV above. Because we did not think taking an "average" or "maximum" value represented the various aspects of risks described in these previous sections, we opted to take "medium" values across the board.

Therefore, we rate this as medium-strength evidence with medium agreement, from primarily self-reporting, and therefore have medium confidence that risk in this area is medium.

\section{VI.B. Risk to a Valued Ecosystem Service}

Ecosystem services at SLBE are created by wildlife viewing and recreation at SLBE; therefore, again the risk here reflects those described above, and we opted to take "medium" values across the board. In addition, we note that these services could be disrupted if areas need to be closed for fire management if needed to maintain lupine - which we deem an additional "medium" risk. 
Therefore, we rate this as medium-strength evidence with medium agreement, from primarily self-reporting, and therefore have medium confidence that risk in this area is medium.

We also speculate that managed relocation of the KBB has the potential to enhance these services by allowing visitors the opportunity to view a rare butterfly. We speculate that the public would support such an action and that it could potentially increase tourism to the park by a small amount, thereby widening the number of people benefiting from the services at SLBE. However, these benefits would depend on the success of the managed relocation and potential risks either being deemed acceptable or not coming to pass. Finally, we speculate that such an action could provide a valuable point of interaction for dialogue with the public about endangered species and climate change impacts and adaptation.

VI.C. Other risk

None.

\section{Conclusion}

This case study underlines the importance of considering species interactions when assessing managed relocation (Figure C-6). Multi-species managed relocation proposals may be especially common because ecological specialization - for example on a host plant - may limit unaided range shifts (Hellmann et al., 2012), making specialist species (and the species they depend upon) likely candidates for managed relocation. We initially suspected that managed relocation of the KBB would provide an example of a relatively "benign" action, since KBB itself has been successfully relocated before and is unlikely to create large changes at the recipient site. However, the assessment process has revealed some areas of high risk, primarily due to the associated managed relocation of lupine. This is despite the fact that we have only completed a preliminary assessment of risks from lupine, opting to focus on KBB for the sake of the case study. An actual assessment process would require more thorough focus on lupine, perhaps resulting in additional components of risk we have not covered here. In the near-term, one way to reduce the ecological risk of a KBB managed relocation would be to choose a recipient site where lupine is already present. However, in the long term, both species will likely need to shift ranges. 


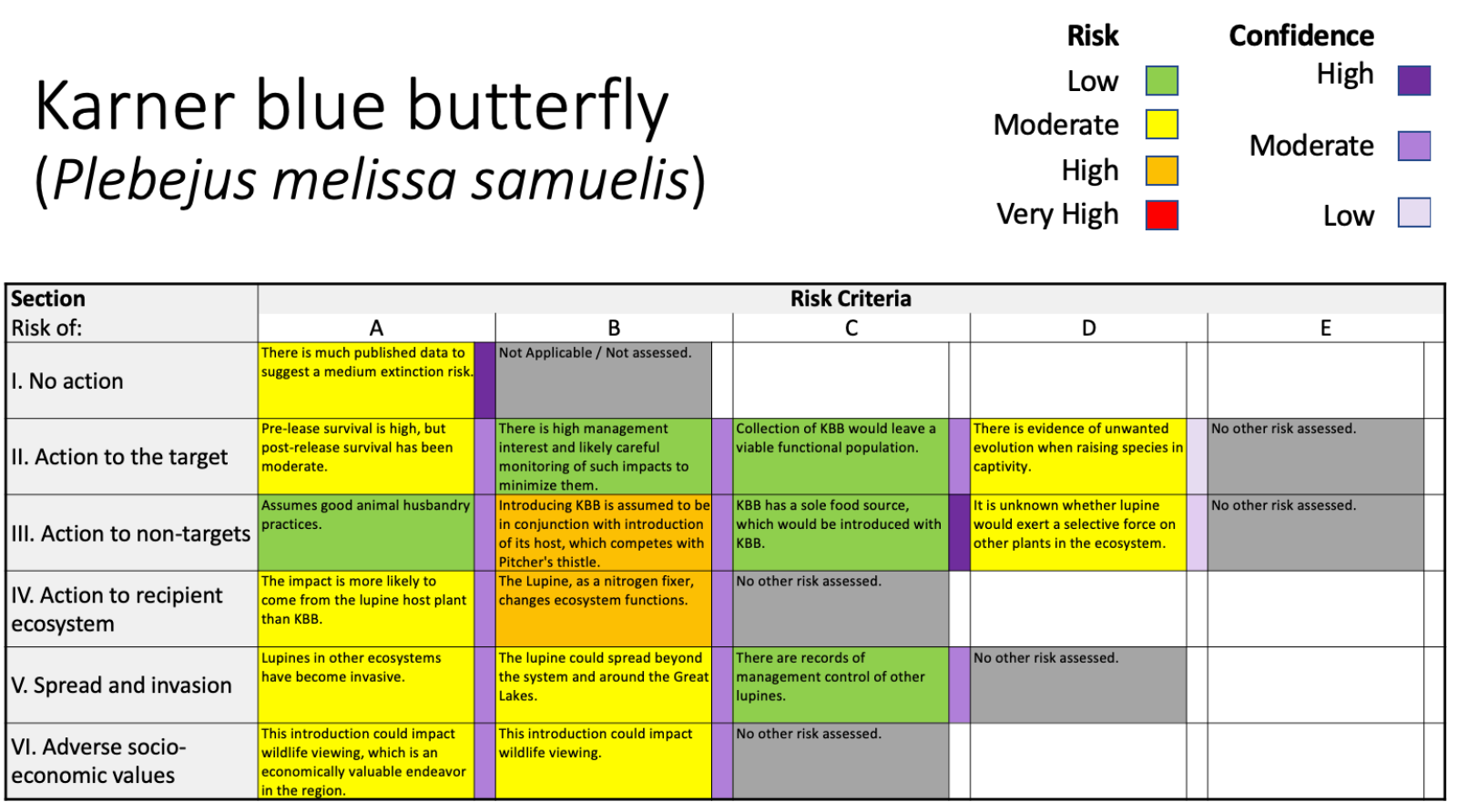

Figure C-6. Risk visualization table of the managed relocation of Karner blue butterfly (Plebejus melissa samuelis).

\section{Literature Cited}

Bristow, L. V. 2017. Effects of warming on the endangered Karner blue butterfly: An exploration of the sensitivity of life history stages and traits. MS Thesis. University of Notre Dame, Indiana.

Crone, E. E., D. Pickering, C. B. Schultz. 2007. Can captive rearing promote recovery of endangered butterflies? An assessment in the face of uncertainty. Biological Conservation 9:1-10. https://doi.org/10.1016/j.biocon.2007.06.007

Dirig, R. 1994. Historical notes on wild lupine and the Karner Blue Butterfly at the Albany Pine Bush, New York in D. A. Andow, R. J. Baker, C. Lane, editors, Karner Blue Butterfly: A Symbol of a Vanishing Landscape. University of Minnesota Minnesota Agricultural Experiment Station, St. Paul, Minnesota.

Fuller, S. G. 2008. Population dynamics of the endangered Karner blue butterfly (Lycaeides melissa samuelis Nabokov). PhD Disseration. State University of New York, Syracuse, NY.

Grundel, R. 2013. USFWS Endangered Species Permit TE10887A Annual Report. Available at: https://www.fws.gov/midwest/endangered/permits/index.html.

Hällfors, M. H., J. Liao, J. D. K. Dzurisin, R. Grundel, M. Hyvärinen, K. Towle, G. C.Wu, and J. J. Hellmann. 2016. Addressing potential local adaptation in species distribution models: implications for conservation under climate change. Ecological Applications 26:1154-1169.

Halpern, S. L. 2003. Evaluating the potential for adaptation to climate change in Lupinus perennis. $\mathrm{PhD}$ Dissertation. University of Minnesota. 
Hellmann, J. J. 2002. The effect of an environmental change on mobile butterfly larvae and the nutritional quality of their hosts. Journal of Animal Ecology 71:925-936.

Hellmann, J. J., K. M. Prior, and S. L. Pelini. 2012. The influence of species interactions on geographic range change under climate change. Annals of the New York Academy of Sciences 1249:18-28. https://doi.org/10.1111/j.1749-6632.2011.06410.x

Hoving, C. L., Y. M. Lee, P. J. Badra, and B. J. Klatt. 2013. Changing climate, changing wildlife: a vulnerability assessment of 400 species of greatest conservation need and game species in Michigan. Wildlife Division Report No. 3564:1-82.

Knutson, R.L., and J. R. Kwilosz. 1999. Movement patterns and population characteristics of the Karner Blue Butterfly (Lycaeides melissa samuelis) at Indiana Dunes National Lakeshore. Natural Areas Journal 19:109-120.

Kwilosz, J. R., and R. L. Knutson,. 1999. Prescribed fire management of Karner blue butterfly habitat at Indiana Dunes National Lakeshore. Natural Areas Journal 19:98-108.

Lee, T. D., M. G. Tjoelker, R. B. Reich, and M. P. Russelle. 2003. Contrasting growth response of an N2-fixing and non-fixing forb to elevated CO2: Dependence on soil N supply. Plant and Soil 255:475-486.

Maron, J. L., and P. G. Connors. 1996. A native nitrogen-fixing shrub facilitates weed invasion. Oecologia 105:302-312.

McLaughlin, J. F., J. J. Hellmann, C. L. Boggs, and P. R. Ehrlich. 2002. Climate change hastens population extinctions. Proceedings of the National Acadamy of Science USA 99:6070-6074.

NatureServe,.2019. NatureServe Explorer: An online encyclopedia of life. Version 7.1. [WWW Document]. Arlington, Virginia. Available at: http://explorer.natureserve.org (accessed 27 March 2019).

Nice, C. C., Z. Gompert, M. L. Forister, and J. A. Fordyce. 2009. An unseen foe in arthropod conservation efforts: The case of Wolbachia infections in the Karner blue butterfly. Biological Conservation 142:3137-3146.

Patterson, T. A., R. Grundel, J. D. K. Dzurisin, R. L. Knutson, R.L., and J. J. Hellmann. 2020. Evidence of an extreme weather-induced phenological mismatch and a local extirpation of the endangered Karner blue butterfly. Conservation Science and Practice 2.

Pelini, S. L., J. A. Keppel, A. E. Kelley, and J. J. Hellmann. 2010. Adaptation to host plants may prevent rapid insect responses to climate change. Global Change Biology 16:2923-2929. https://doi.org/10.1111/j.1365-2486.2010.02177.x 
Pickart, A. J., L. M. Miller, and T. E. Duebendorfer. 1998. Yellow bush lupine invasion in northern California coastal dunes I. Ecological impacts and manual restoration techniques. Restoration Ecology 6:59-68. https://doi.org/10.1046/j.1526-100x.1998.00618.x

Pickens, B. A., and K. V. Root. 2007. An assessment of ant abundance in oak savanna, the evaluation of two butterfly reintroduction techniques, and recommendations for the Karner Blue Butterly in Ohio. A Report to the Ohio Department of Natural Resources, U. S. Fish and Wildlife Service, and the Nature Conservancy (OH Chapter).

Pickens, B. A., and K. V. Root. 2009. Behavior as a tool for assessing a managed landscape : a case study of the Karner blue butterfly. Landscape Ecology 24:243-251. https://doi.org/10.1007/s10980-008-9302-z

Reznicek, A. A., E. G. Voss, and B. S. Walters. 2011. Michigan Flora Online. University of Michigan. Available at: https://www.michiganflora.net/species.aspx?id=1327 (accessed 27 June 2019).

Schneider, A. C., T. D. Lee, M. A. Kreiser, and G. T. Nelson. 2014. Comparative and interactive effects of reduced precipitation frequency and volume on the growth and function of two perennial grassland species. International Journal of Plant Sciences 175:702-712.

U.S. Fish and Wildlife Service (USFWS). 2003. Final recovery plan for the Karner blue butterfly (Lycaeides melissa samuelis). Fort Snelling, Minnesota. Available at: https://ecos.fws.gov/docs/recovery_plan/030919.pdf.

U.S. Fish and Wildlife Service (USFWS). 2012. Karner blue butterfly 5-year review: summary and evaluation. New Franken, Wisconsin. Available at: https://ecos.fws.gov/ecp0/profile/speciesProfile?spcode=I00F.

Valtonen, A., J. Jantunen, and K. Saarinen. 2006. Flora and lepidoptera fauna adversely affected by invasive Lupinus polyphyllus along road verges. Biological Conservation 133:389-396. https://doi.org/10.1016/j.biocon.2006.06.015

Webb, L. 2010. Propagation handbook for the Karner blue butterfly, Lycaeides melissa samuelis. Concord, New Hampshire. 


\section{Case Study: Risk Assessment of the Managed Relocation of Giant Sequoia (Sequoiadendron giganteum)}

Aviv Karasov-Olson and Mark W. Schwartz (University of California, Davis)

\section{Hypothetical Action}

We completed a risk assessment of the managed relocation of giant sequoia (Sequoiadendron giganteum) from existing groves to higher elevations within Sequoia and Kings Canyon National Parks. We envision a management proposal to collect seed from trees in existing groves within the parks, raise the seeds to seedlings in national park facilities within the park, and plant the seedlings in meadows that are higher, cooler, and wetter than existing groves (Figure C-7). These meadows would be chosen based on a climate model of the capacity for the trees to survive for the longest possible period of time given climate change. The project would entail the loss of existing natural meadow habitat to make sequoia groves and may require additional follow-up management actions such as prescribed fire to ensure continued recruitment and growth.

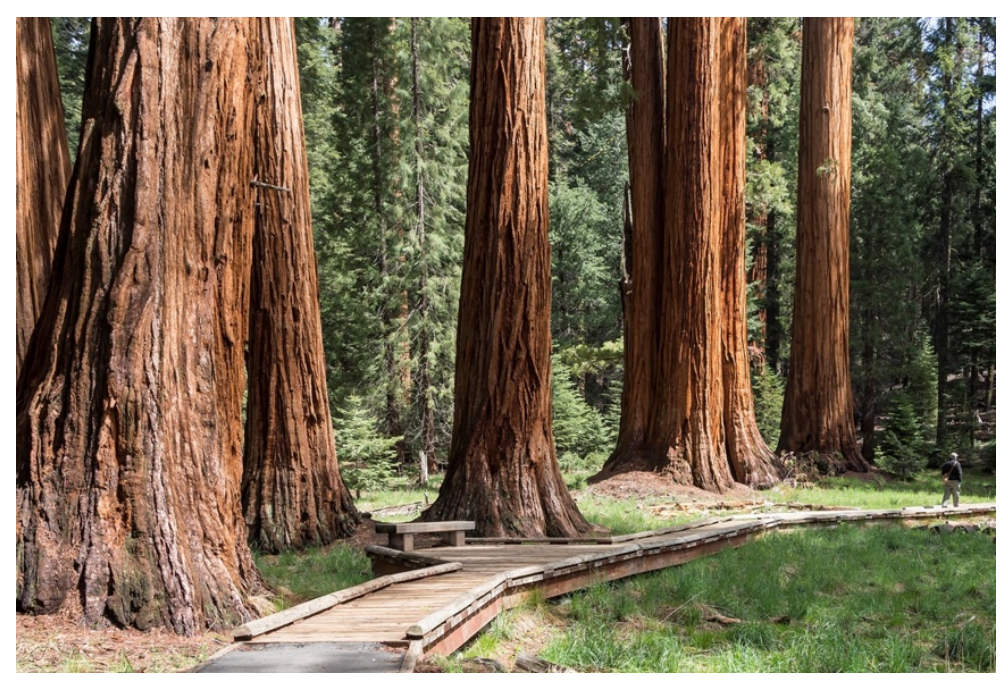

Figure C-7. Giant sequoias along a trail in Sequoia and Kings Canyon National Parks. Photo credit: NPS

\section{Background}

There are $\sim 75$ giant sequoia groves distributed along the western slope of the Sierra Nevada within a narrow elevational band between $1400 \mathrm{~m}$ to $2150 \mathrm{~m}$. They prefer well-drained sandy soils and grow best in more mesic environments (Weatherspoon 1990), which is why current, high elevation meadows are the ideal recipient ecosystems for the managed relocation of this species. Seeds are often wind-dispersed and may also be indirectly dispersed through caching by Douglas squirrels (Tamiasciurus douglasii; Weatherspoon 1990). Giant sequoias are also shade intolerant throughout their lives; fire serves to create gaps in the canopy that foster sequoia establishment and growth (Weatherspoon 1990, Aune 1994). 


\section{Risk Assessment}

$\underline{\text { Section I. Risk of No Managed Relocation Action }}$

\section{I.A. Risk of No Action to the Target}

Giant sequoia appears to be moderately at risk of extirpation of natural groves primarily due to predicted low recruitment in the face of climate change, rather than adult tree mortality (York et al. 2013b). Therefore, extinction risk is not imminent and natural groves are likely to persist in place for some time. An increase in droughts and reduced snowpack are likely the changes that will most negatively affect giant sequoia (York et al. 2013b). Under future climate models, snowpack is predicted to shrink in size and snow melt may also come earlier in the spring reducing water availability during dry summers. This poses a problem because giant sequoia are reliant on snow melt to recharge soil moisture, which is a key habitat requirement for growth (Rundel 1972).

During the recent (2012-2016) severe drought in California, giant sequoia experienced higher rates of foliage dieback, though adult tree mortality was very low ( $<1 \%$; Stephenson et al. 2018).

Additionally, foliage dieback was greatest at lower elevations (Stephenson et al. 2018), supporting a managed relocation action to higher elevations where overall survival and health may be greater. Increasing severity of droughts may magnify the impacts of previously non-lethal factors such as cambial damage from severe fires, high abundances of Phloeosinus bark beetles, and low baseline canopy water content (Nydick et al. 2018, Paz-Kagan et al. 2018).

While giant sequoias often employ many strategies, such as drought-induced foliage dieback, the adaptive capacity of this species is low. Genetic diversity is low overall, and lower in northern groves compared to southern groves (Dodd and DeSilva 2016). This is particularly troubling as northern groves may serve as a source for natural range shifts to higher latitudes and elevations in response to climate change (Dodd and DeSilva 2016).

Together these appear to result in a moderate risk of loss of natural populations with relatively high confidence based on published data on the target species for which there is high agreement and robust evidence. Extinction risk of the entire species, however, is quite low. There are numerous known botanical garden collections and individuals introduced in hundreds of locations around the world. Although the wild population has a limited number of groves, the gene pool is wellrepresented in terms of numbers and center of origin in horticultural populations.

\section{I.B. Risk of No Action to the Recipient Ecosystem}

The purpose of the managed relocation action is for the conservation of giant sequoia. Therefore, the risk of no action on the recipient ecosystem is not applicable.

\section{$\underline{\text { Section II. Risks of Action Associated with the Target }}$}

\section{II.A. Risk of Action to the Relocated Individuals}

The most vulnerable period for giant sequoia survival is during the first year, when long, dry summers can limit seedling establishment (York et al. 2013a). However, following establishment, sequoia survival is around 90\% during the first few decades (York et al. 2013a). Given adequate disturbance (ideally fire), seedling survival during the first year can still be high ( $75 \%$; York et al. 
2006, 2011). One potential limitation to establishment is that soils at higher elevations may not be well-suited for giant sequoia (Stephenson 1996), though it is not clear to what degree this would inhibit survival or growth of closely managed relocated individuals.

There is also ample anecdotal evidence of these trees being planted in various climates around the world. Planting these upslope, in current meadow environments, should be eminently feasible and successful, particularly with horticultural attention to early establishment.

If the managed relocation action is well-timed and accompanied by appropriate management actions, the risk of no survival of the target is low with relatively high confidence based on published data on the target species which provides high agreement among robust evidence.

\section{II.B. Risk That the Target Source Population Cannot Withstand Diminished Numbers}

Seed production of giant sequoia is high (Weatherspoon 1990). As a long-lived tree, harvesting seed for transplantation should have no long-term impact on the established populations and their capacity for recruitment. Individual trees can produce as many as 300,000 seeds per year (York et al 2013b). Collecting a moderate amount of seed (e.g., 1000's) will not substantively impact the seed pool. The project would not remove established individuals.

The managed relocation action poses low risk to the source population with high confidence based on high agreement with moderate evidence. This risk score is based on published natural history data on the target and a self-reported understanding of the transplantation process.

II.C. Risk That Removing Individuals of the Target Will Negatively Impact a Key Function in the Source Ecosystem

As above, harvesting seed for transplantation should have no long-term impact on the established populations and their capacity for recruitment and established mature trees would not be removed from a wild source population. Therefore, the risk to the source ecosystem is low with high confidence (despite low evidence strength) based on self-reported understanding of the biology of giant sequoia.

\section{II.D. Risk of Causing Undesired Evolution in the Target}

Overall genetic diversity of giant sequoias is low relative to other trees (Fins and Libby 1982). Some critical traits differ between natural sequoia groves. For example, the flushing dates between southern and northern groves is different (Fins and Libby 1982). The proposed movement, however, is highly local, and the relocated population can be large and contain a solid sampling of standing genetic variation. The recipient location also has similar background environment and selective forces to the source ecosystem.

Additionally, giant sequoias have been planted in many locations and environments around the globe without any dramatic, apparent evolutionary changes to the populations (N. Stephenson, personal communication, unpublished reports form Sierra Pacific Industries).

The risk of undesired evolution in the relocated population is low, though confidence is moderate given moderate agreement and limited evidence strength of published data. 
Section III. Risks of Action to Non-targets within the Recipient Ecosystem

III.A. Risk of Target Transmitting Novel Disease or Associated Pest

Giant sequoias are susceptible to Annosus root rot (Heterobasidion annosum) and Armillarea root rot (Armillaria mellea), both of which can be transmitted between neighboring tree root systems (Stephenson 1996, York et al. 2013b). Infections from the former occur more frequently when spread from white fir (Abies concolor), rather than giant sequoia acting as the primary source of the infection (York et al. 2013). Armillarea root rot, on the other hand, can be spread to other species such as black oak (Quercus kelloggii), which are preferred hosts (York et al. 2013b). Phloesinus bark beetles have also been detected on giant sequoia with few mortality events (Stephenson et al. 2018).

While giant sequoias are known to be associated with these pests and pathogens, the transplanted seeds can be collected from on-site, grown on-site in species-specific facilities, and cleared of infestation prior to introduction in the release site. The process is highly unlikely to introduce any horticultural disease agents or pests.

Therefore, risk of the target transmitting a novel disease or pest to other species within the recipient ecosystem is low with high confidence based on moderate agreement among robust published data on the target.

\section{III.B. Risk of Competitive Interaction Negatively Affecting the Distribution or Abundance of Non-targets}

This managed relocation action would require the conversion of select high elevation meadows into sequoia forest ecosystems, essentially destroying the meadows. So, by design this action would severely negatively impact all non-target, meadow species in the introduction site.

Should the relocated individuals interact with surrounding forests at high elevations, there may also be negative competitive interactions. Giant sequoias generally perform better, growing larger and taller, when grown sympatrically with other conifers (ponderosa pine, Pinus ponderosa; Jeffrey pine, P. jeffreyi; sugar pine, P. lambertiana; Douglas-fir, Pseudotsuga menziesii; white fir, Abies concolor; and red fir, A. magnifica; Kitzmiller and Lunak 2012). As seedlings, they may experience greater competition for light, as evidenced by improved growth following shrub removal (Heald and Barrett 1999). As mature trees, there may be greater competition for soil moisture (York et al. 2011), though this evidence primarily focused on intra-specific competition. Giant sequoias also require periodic disturbance, either in the form of low severity fire or shrub removal, for growth (York et al. 2006, 2015). Although these disturbances do not constitute direct competition, they may decrease the abundance of non-target species within the release site.

The meadows are also conservation targets. Planning can help identify meadows most likely to be lost to climate change, or of least conservation value, to minimize these negative impacts. The overall impact on meadow ecosystems may be low based on a plan to create a dozen or so new groves and there being hundreds of meadows within the system.

There is high risk of competitive interactions negatively impacting non-target species, particularly meadow species, with moderate confidence based on moderate agreement. This risk score is selfreported and based on some moderate published data on the species. 
III.C. Risk of Consumptive Effects Reducing the Abundance or Distribution of Non-targets

This risk is not applicable to giant sequoia because it is a vascular plant.

III.D. Risk of Driving Undesired Evolution in Non-targets

Giant sequoias are more likely to directly affect the growth and abundance of non-target species. Anecdotally, there is no evidence of evolutionary changes in non-target species in location where giant sequoias have been previously transplanted.

There is a low risk that relocated sequoia will drive undesirable evolution in non-targets. Confidence in this assessment is low as it is mostly self-reported and there is little published data on the topic.

Section IV. Risks of Action to Non-target, Higher Order Attributes of the Recipient Ecosystem

IV.A. Risk of Indirect and Negative Impacts on Ecosystem Structure

Changing a meadow to a forest would change the ecosystem and its food web entirely.

The risk of indirect and negative impacts on the food web is, therefore, very high, with high confidence. The evidence is self-reported; however it is likely that published information could be garnered to show that when planting trees in a meadow, the meadow is converted to a forested ecosystem.

\section{IV.B. Risk of Changing Ecosystem Function}

As a result of the introduction of giant sequoia, the ecosystem function of the release site would fundamentally change because the ecosystem would be converted from a meadow to a forest.

Other potential changes may result from transplanting giant sequoia. Giant sequoias change the microclimate of areas surrounding individual trees by reducing summertime surface temperature, near-ground air temperature, and surrounding $\mathrm{CO}_{2}$ concentrations (Eckmann et al. 2018). Giant sequoias can also alter small wind movements (Eckmann et al. 2018). As described above, SEGI requires ample soil moisture for growth (Weatherspoon 1990). The establishment of a large, relocated population of giant sequoia may also cause changes to ecosystem function with respect to carbon storage and water flows.

Additionally, the reliance of giant sequoia on fire (Weatherspoon 1990) could lead to changes in ecosystem function. The National Park Service has historically used prescribed fires to manage native groves (Demetry 1998, van Wagtendonk 2007). Young SEGI stands in particular would benefit from low-severity prescribed fires (York et al. 2013a). Increasing the frequency or changing the intensity of fires within the recipient ecosystem may cause longer lasting changes to ecosystem function.

The risk of changing ecosystem function is very high with high confidence, based on high agreement and moderate published data on the target. 
$\underline{\text { Section V. Risks Associated with Biological Invasion }}$

\section{V.A. Risk of Invasion Within the Intended Recipient Ecosystem}

Giant sequoias grow very slowly and recruit minimally. They can produce 300,000 seeds per year, though most do not germinate (York et al. 2013b). Seeds can disperse short distances of up to $400 \mathrm{~m}$ (York et al. 2013b). Giant sequoias have relatively narrow environmental requirements including greater than $50 \%$ of precipitation falling as snow (York et al. 2013b) and greater soil water-holding capacity (Lutz et al. 2010).

Therefore, there is a low risk of invasion within the recipient location, with high confidence, based on high agreement among modest published data on the target.

\section{V.B. Risk of Invasion Beyond the Recipient Ecosystem}

As mentioned above, giant sequoias have a relatively low dispersal ability (York et al. 2013b). Also, grove establishment appears to be a very limiting constraint. Under the scenario of moving sequoia upslope, there is limited area surrounding the recipient location for the species to invade. In fact, the managed relocation action would be taken because of a limited capacity for this species to naturally expand its range upslope.

Additionally, there are not reported cases of the species spreading and becoming invasive; more than a century of study of sequoia groves in the Sierra's has resulted in no observations of spontaneous grove establishment.

Therefore, there is a low risk of invasion beyond the recipient location, with high confidence, based on high agreement among modest published data on the target and the conditions of this managed relocation action.

\section{V.C. Risk That the Target Cannot Be Controlled Should It Become Invasive}

Giant sequoia seeds are well-adapted for wind dispersal (Weatherspoon 1990), which presents the primary challenge in controlling the relocated population. However, the relatively slow growth rate of the species would make it easy to control. A decision to reverse the process could easily be accomplished successfully.

With high confidence, based on high agreement, and despite limited evidence, there is low risk that the target could not be controlled should it become invasive.

\section{$\underline{\text { Section VI. Risks Associated with Socio-economic Values }}$}

\section{VI.A. Risk to a Culturally or Economically Important Species}

In this case study, the target species is likely to be the more societally-valued species. Therefore, we are self-reporting that there is a low risk to a societally-valued species, with moderate confidence due to high agreement and limited evidence.

\section{VI.B. Risk to a Valued Ecosystem Service}

The destruction of meadows for the creation of new sequoia groves might result in a stronger social risk where meadows are more highly valued (e.g., Zumwalt Meadows offers popular hiking within 
Sequoia and Kings Canyon National Parks). It is less clear how meadows contribute to the overall hydrological system and how changing from a meadow to a forest would alter the sub-surface water balance. Sequoias would transpire more water out of the system, but also shade the system more than meadows. Also, the sub-surface hydrology is complex in the Sierra Nevada. Although meadows, as a whole, are thought to contribute substantially to ecosystem water recharge and maintenance of stream flows, it is less clear how changing a small suite of meadows would affect the overall hydrology of the ecosystem

Therefore, we are self-reporting that there is a low risk to a valued ecosystem service, though the confidence is low and dependent on perceived value of meadow ecosystems.

\section{Conclusion}

We considered this case study in an effort to understand the risks associated with moving a large, slow-growing vascular plant that greatly impacts its associated ecosystems (Figure C-8). The risk posed to giant sequoias and their source ecosystem are low, particularly if the relocated individuals are young seedlings, initially grown in a species-specific facility. Therefore, the implementation strategies greatly impact the risk of action associated with the target. While risk to specific nontargets within the recipient ecosystem are low to moderate, managed relocation of giant sequoia poses a much greater risk to the recipient ecosystem overall. This action would likely require moving giant sequoia into current alpine meadows and would result in a conversion of the whole recipient ecosystem. There was moderate confidence in this ecosystem-level risk due to limited available evidence that studied this potential result; this conclusion was based on some assumptions of ecosystem change and could be enhanced by future research. The decision to relocate giant sequoia upslope would therefore depend on weighing the benefits and necessity of relocating giant sequoia against the consequential loss of one to several alpine meadows. 


\section{Giant sequoia (Sequoiadendron giganteum)

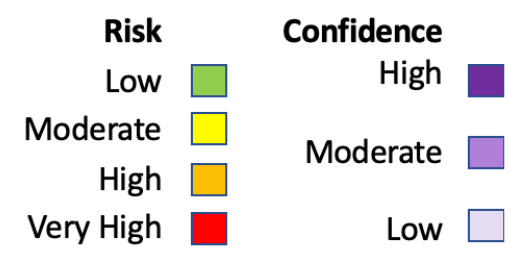

\begin{tabular}{|c|c|c|c|c|c|}
\hline Section & & & Risk Criteria & & \\
\hline Risk of: & A & B & C & D & $\mathrm{E}$ \\
\hline I. No action & Numerous collections. & Not assessed. & & & \\
\hline II. Action to the target & $\begin{array}{l}\text { Establishment of } \\
\text { plantings well- } \\
\text { documented. }\end{array}$ & $\begin{array}{l}\text { Numerous sources } \\
\text { available. }\end{array}$ & $\begin{array}{l}\text { Collection will not harm } \\
\text { ecosystem. }\end{array}$ & $\begin{array}{l}\text { Highly local proposed } \\
\text { movement. }\end{array}$ & No other assessed. \\
\hline III. Action to non-targets & $\begin{array}{l}\text { Within-park seed } \\
\text { collection limits disease } \\
\text { potential. }\end{array}$ & $\begin{array}{l}\text { Grove establishment will } \\
\text { destroy meadow } \\
\text { species. }\end{array}$ & $\begin{array}{l}\text { Allelopathy not } \\
\text { assessed. }\end{array}$ & $\begin{array}{l}\text { Non-target species } \\
\text { evolution not seen as a } \\
\text { problem. }\end{array}$ & No other assessed. \\
\hline $\begin{array}{l}\text { IV. Action to recipient } \\
\text { ecosystem }\end{array}$ & $\begin{array}{l}\text { Ecosystem conversion is } \\
\text { intended. }\end{array}$ & $\begin{array}{l}\text { Sequoia is landscape } \\
\text { species. }\end{array}$ & No other assessed. & & \\
\hline V. Spread and invasion & $\begin{array}{l}\text { Species grows slowly } \\
\text { and spreads little. }\end{array}$ & $\begin{array}{l}\text { De novo grove } \\
\text { establishment has not } \\
\text { happened. }\end{array}$ & $\begin{array}{l}\text { Reversibility: Species } \\
\text { could be removed } \\
\text { through timber harvest. }\end{array}$ & No other assessed. & \\
\hline $\begin{array}{l}\text { VI. Adverse socio- } \\
\text { economic values }\end{array}$ & $\begin{array}{l}\text { Many meadows contain } \\
\text { locally restricted species } \\
\text { of significance. }\end{array}$ & $\begin{array}{l}\text { Destroys meadows, } \\
\text { though sequoia may be } \\
\text { more societally-valued. }\end{array}$ & No other assessed. & & \\
\hline
\end{tabular}

Figure C-8. Visualization of overall risk of managed relocation of giant sequoia.

\section{Literature Cited}

Aune, P. S. 1994. Proceedings of the symposium on giant sequoias: their place in the ecosystem and society; June 23-25; Visalia, CA. Page General Technical Report PSW-GTR-151. Albany, California.

Demetry, A. 1998. A natural disturbance model for the restoration of giant forest village, Sequoia National Park. Pages 142-159 in W.R. Keammerer and E.F. Redente, editors. Proceedings of High Altitude Revegetation Workshop, No. 13 Colorado Water Resources Research Institute, Information Series No. 89, Fort Collins, Colorado

Dodd, R. S., and R. DeSilva. 2016. Long-term demographic decline and late glacial divergence in a Californian paleoendemic: Sequoiadendron giganteum (giant sequoia). Ecology and Evolution $6: 3342-3355$.

Eckmann, T., A. Morach, M. Hamilton, J. Walker, L. Simpson, S. Lower, A. Mcnamee, A. Haripriyan, D. Castillo, S. Grandy, and A. Kessi. 2018. Measuring and modeling microclimate impacts of Sequoiadendron giganteum. Sustainable Cities and Society 38:509-525.

Fins, L., and W. L. Libby. 1982. Population variation in Sequoiadendron: seed seedling studies, vegetative propagation, and isozyme variation. Silvae Genetica 31:102-110.

Heald, R. C., and T. M. Barrett. 1999. Effects of planting density on early growth of giant sequoia (Sequoiadendron giganteum). Western Journal of Applied Forestry 14:65-72. 
Kitzmiller, J. H., and G. Lunak. 2012. Growth of giant sequoia compared to ponderosa pine and other mixed-conifers in California plantations. Western Journal of Applied Forestry 27:196-204.

Lutz, J. A., J. W. van Wagtendonk, and J. F. Franklin. 2010. Climatic water deficit, tree species ranges, and climate change in Yosemite National Park. Journal of Biogeography 37:936-950.

Nydick, K. R., N. L. Stephenson, A. R. Ambrose, G. P. Asner, W. L. Baxter, A. J. Das, T. Dawson, R. E. Martin, and T. Paz-Kagan. 2018. Leaf to landscape responses of giant sequoia to hotter drought: an introduction and synthesis for the special section. Forest Ecology and Management 419-420:249-256.

Paz-Kagan, T., N. R. Vaughn, R. E. Martin, P. G. Brodrick, N. L. Stephenson, A. J. Das, K. R. Nydick, and G. P. Asner. 2018. Landscape-scale variation in canopy water content of giant sequoias during drought. Forest Ecology and Management 419-420:291-304.

Rundel, P. W. 1972. Habitat restriction in giant sequoia: the environmental control of grove boundaries. The American Midland Naturalist 87:81-99.

Stephenson, N. L. 1996. Ecology and management of giant sequoia groves. in Sierra Nevada Ecosystem Project: final report to Congress, volume II. Assessments and scientific basis for management options. Centers for Water and Wildland Resources Report no. 37. Davis, California.

Stephenson, N. L., A. J. Das, N. J. Ampersee, K. G. Cahill, A. C. Caprio, J. E. Sanders, and A. P. Williams. 2018. Patterns and correlates of giant sequoia foliage dieback during California's 2012 - 2016 hotter drought. Forest Ecology and Management 419-420:268-278.

van Wagtendonk, J. W. 2007. The history and evolution of wildland fire use. Fire Ecology 3:3-17.

Weatherspoon, C. P. 1990. Sequoiadendron giganteum (Lindl.) Buchholz giant sequoia. Silvics of North America 1:552-562.

York, R. A., J. J. Battles, A. K. Eschtruth, and F. G. Schurr. 2011. Giant sequoia (Sequoiadendron giganteum) regeneration in experimental canopy gaps. Restoration Ecology 19:14-23.

York, R. A., R. C. Heald, and J. J. Battles. 2006. Release potential of giant sequoia following heavy suppression: 20-year results. Forest Ecology and Management 234:136-142.

York, R. A., J. M. Louen, and A. C. Thomson. 2015. Growth response of massive Sequoiadendron giganteum trees to mechanical treatments. Forest Science 61:959-965.

York, R. A., K. L. O’Hara, and J. J. Battles. 2013a. Density effects on giant sequoia (Sequoiadendron giganteum) growth through 22 years: implications for restoration and plantation management. Western Journal of Applied Forestry 28:30-36.

York, R. A., N. L. Stephenson, M. Meyer, S. Hanna, T. Moody, A. C. Caprio, and J. J. Battles. 2013b. A natural resource condition assessment for Sequoia and Kings Canyon National Parks: 
Appendix 11 - Giant sequoia. Natural Resource Report NPS/SEKI/NRR-2013/665.11. Fort Collins, CO. 


\section{Case Study: Risk Assessment of the Managed Relocation of Pitcher's Thistle (Cirsium pitcheri)}

Mark W. Schwartz and Aviv Karasov-Olson (University of California, Davis)

\section{Hypothetical Action}

As a hypothetical case study, we address the notion of moving Pitcher's thistle (Cirsium pitcheri (Eaton) Torrey \& Gray, Asteraceae), a federally endangered narrow endemic with declining populations, from its current locations to a vaguely defined region northward (Figure C-9). Given that the current distribution appears to reach the northern edge of the Great Lakes lakeshore in the US, and we are uncertain of the distribution of dunes north of its current distribution, this managed relocation project may include the augmentation of current populations through managed gene flow, moving individuals from more southerly populations into more northerly populations. We treat that as a secondary and less likely action. This latter would adjust (mostly downward) some of our assessment of risk.

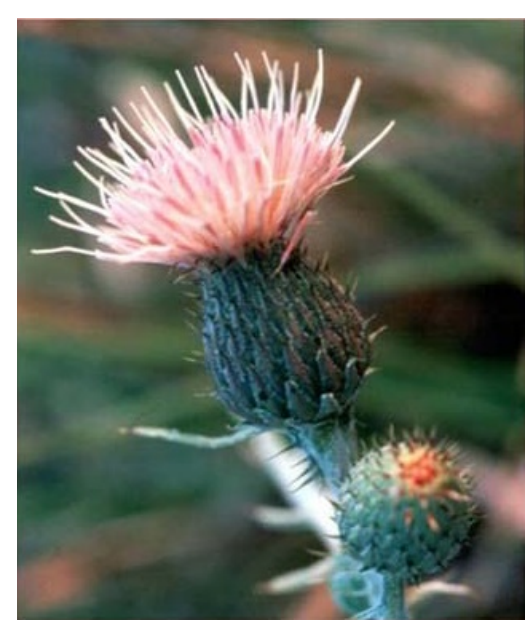

Figure C-9. Pitcher's thistle. Photo credit: USFWS

We do not envision any particular proposed scenario for managed relocation, and none has been proposed to our knowledge. The proposition of a climatic rescue for the species is made complex by the fact that the distribution extends into Canada with about 30 populations north of the US border (Phillips and Maun, 1996, D'Ulisse and Maun 1996, Chen and Maun 1998, Promaine 1999, Nantel et al. 2018). A managed relocation proposal for Pitcher's thistle may need to be by international agreement.

\section{Background}

We chose Pitcher's thistle (Cirsium pitcheri), an endangered and narrowly restricted endemic, as a second plant case study. We selected this species based on four criteria. First, it is a federally restricted species that appears to be declining and may be at risk to climate change driven extirpations (Bowles et al. 1993, Halsey et al. 2016, Nantel et al. 2018). Second, it has historical occurrences on National Park Service lands (Indiana Dunes National Park) (NPS 2015) and a potential managed relocation site within a National Park (Apostle Islands National Lakeshore). 
Third, it is a species with a sharply contrasting life history from the other plant case study chosen (giant sequoia (Sequoiadendron giganteum)). Finally, there is a rich literature on the threats to the species persistence.

Pitcher's thistle is a short-lived monocarpic herbaceous species (Bowles et al. 1993, USFWS 2002, NPS 2015). Pitcher's thistle is endemic to dune environments along the shores of three of the Great Lakes (Superior, Michigan, Huron; USFWS 2002). At present there appear to be no extant populations within Indiana Dunes National Park (NPS 2015). A series of population viability studies have concluded that although individual populations are declining and each have a relatively short projected lifespan, the collected metapopulation appears stable over the next 100 years given unchanged climate (Bell et al. 2013, Jolls et al. 2015, Halsey et al. 2016, Nantel et al. 2018). The species appears vulnerable to increases in temperature (Staehlin and Fant 2015) and restoration efforts have noted a sensitivity to low precipitation in new population establishment (Halsey et al. 2017a). Primary non-climatic threats to existing populations appear to be invasive species that colonize these dune habitats (Emery et al. 2013, Girdler et al. 2016, Girdler and Radke 2006, LeichtYoung and Pavlovic 2012, Rand et al. 2015), intensive herbivory by white-tailed deer (Phillips and Maun 1996), as well as a biocontrol agent released to attack non-native thistles, which has spread onto Pitcher's thistle (Hakes and Meunier 2018, Havens et al. 2012, Louda et al. 2005a,b). This weevil reduces plant fitness in Pitcher's thistle (Havens et al. 2012).

The risk assessment for Pitcher's thistle is greatly informed by research attention to this species and its ecology. First, a series of genetic studies have characterized the within and between population variation in Pitcher's thistle (Fant et al. 2013, 2014, Gauthier et al. 2010). Second, several studies have investigated the ecology of this species and its population dynamics (e.g., Marshall 2014, Perumal and Maun 2006, Rudgers et al. 2015, Stanforth et al. 1997). This risk assessment is also substantively informed by a series of experiments restoring Pitcher's thistle into historic locations (Rowland and Maun 2001, Chen and Maun 1999, Halsey et al. 2017a, b, Halsey et al. 2015, Hamze and Jolls 2000). Thus, much is known about the potential for management to establish new populations.

\section{Risk Assessment}

\section{$\underline{\text { Section I. Risk of No Managed Relocation Action }}$}

\section{I.A. Risk of Action to the Target}

Based on a substantial literature on the viability of the species, we assessed that there is a moderate chance of loss of the species in the future. At present there appear to be no individuals within Indiana Dunes National Park, our focal location for managed relocation. The most recent and most sophisticated population models use 17 years of data to show a high probability of persistence in Canada over the next century, albeit without considering climate change (Nantel et al. 2018). However, this same study found a high probability of local extirpation or a $50 \%$ decline in several smaller populations (Nantel et al. 2018). A spatially explicit, individually based model (Halsey et al. 2017b) shows most populations at risk of declining, again not considering the impacts of changing climates. This team also shows the importance of metapopulations in increasing potential species persistence (Halsey et al. 2016), and that restorations can improve metapopulation viability (Halsey 
et al. 2015). This same improvement may be provided by managed relocations as well. Very little is known specifically about climate change impacts, but end of century temperatures are associated with poorer population performance (Staehlin and Fant 2015). Growth performance of experimental populations under end-of-century conditions was correlated with the genetic diversity of the parent populations, indicating the importance of maintaining high genetic diversity in the relocated individuals (Staehlin and Fant 2015).

In addition, there are significant non-climatic threats to the existing populations in the form of habitat displacement by invasive plants, as well as reduced fitness as a consequence of infestation by an introduced biocontrol weevil that has spread onto this species. Marshall (2014) reports increasing numbers of dunes that are stabilizing through greater vegetation cover. Pitcher's thistle requires open active dune formation for seed germination and establishment (Marshal 2014, Chen and Muan 1999).

Together these appear to result in a moderate risk of loss of populations with relatively high confidence based on high agreement among robust published data on the target species.

\section{I.B. Risk of No Action to the Recipient Ecosystem}

The purpose of the managed relocation action is for the conservation of Pitcher's thistle. Therefore, the risk of no action on the recipient ecosystem is not applicable.

Section II. Risks of Action Associated with the Target

II.A. Risk of Action to the Relocated Individuals

There are numerous experiments and previous restoration efforts that provide information about how Pitcher's thistle may respond to the managed relocation action. Transplant size of Pitcher's thistle is related to fecundity (Bell et al. 2013) and may affect survivorship and population establishment (Davies et al. 1999 in Halsey et al. 2017). Higher root-crown diameter in plantings, using plants rather than seed, increased probability of survival upon initial restoration, and ultimate plant sizes were greater on flatter slopes (Halsey et al. 2017). Over the course of ten years, Halsey et al. (2017) observed $48 \%$ survival during the first year following planting. Establishment and survival of C. pitcheri is decreased by the presence of invasive species, Centaurea stoebe subsp. micranthos (Rand et al. 2015), and there is some evidence of feeding by an invasive agricultural pest, Diabrotica undecimpunctata howardi (Marshall 2013). Removal of invasive plants can also increase pollinator visitation to $C$. pitcheri (Baskett et al. 2011). Additionally, there have been several studies examining the proper conditions under which to plant C. pitcheri (e.g., Perumal and Maun 2006). Exclusion of herbivorous insects can dramatically decrease juvenile mortality and increase seed production (Bevill et al. 1999). Other actions can be taken to improve seed germination and seedling emergence of planting using seeds. This includes planting seed at depths between 2 and $6 \mathrm{~cm}$ below the dune surface (Chen and Maun 1999) and artificially scarifying the seeds if natural scarification through freezing and thawing of the surrounding substrate is not possible at the time of planting (Chen and Maun 1998). In established populations, the juvenile phase appears to have the highest mortality rates (D'Ulisse and Maun 1996). Finally, perched dunes may be preferred over lakeshore dunes as they are associated with higher seedling emergence, establishment, and juvenile survivorship (Rand et al. 2015). Perched dunes are those dunes set back from the lakeshore, established under previous, 
higher lake levels. Set back from the lake shore these perched dunes have older soils, support different vegetation, experience different disturbance regimes, and different hydrologic regimes.

Based on extensive previous experience with transplanting this species into restoration sites within the historical range, with high confidence (based on high agreement and robust evidence), we judged the risk to the relocated individuals to be low based on published data on the target species.

\section{II.B. Risk That the Target Source Population Cannot Withstand Diminishes Numbers}

Given extensive attempts at population restoration, we presume that there is a current working knowledge on using seed from wild individuals without diminishing the capacity of that population to persist. However, one study (Halsey et al 2017a) suggests that it may take a massive amount of seed, perhaps 250,000 seeds, to reliably establish a new population. Plants in the wild take four to eight years to flower and set seed (Gijmans et al. 2020). Up to 150 seeds are produced per seed head with larger plants achieving up to 10 seed heads (Gijmans et al. 2020). An invasive weevil attacks seed heads and reduces fecundity (Gijmans et al. 2020). A low fraction of seeds germinates and establish. The consequence is that it is quite likely that small collections used to initiate an offsite nursey would be required to produce a sufficient amount of seed for managed relocation.

As such, we judged the risk to source populations to be moderate based on published data on the target. However, we only assess this risk with moderate confidence due to limited evidence.

II.C. Risk That Removing Individuals of the Target Will Negatively Impact a Key Function in the Source Ecosystem

Since we envision moving greenhouse-produced juveniles or seeds, and not adults, we self-report that the risk to the source population to be low with high confidence based on some published data as well.

\section{II.D. Risk of Causing Undesired Evolution in the Target}

C. pitcheri has relatively low genetic diversity and high levels of inbreeding (Gauthier et al. 2009, Fant et al. 2014), though northern populations tend to have higher genetic diversity (Fant et al. 2014). C. pitcheri exhibits higher levels of inbreeding than $C$. hillii, a congeneric species with similar habitat requirements found in a similar geographic area (Freeland et al. 2010). Fant et al. (2014) compared genetic diversity of native source populations to that of restored populations. While using nearby local sources for the restorations led to higher genetic diversity than those that did not, restored populations had higher levels of inbreeding (Fant et al. 2014). Managed relocation efforts should therefore focus on supporting establishment and successful flowering, as well as considering the number and genetic diversity of relocated individuals. Additionally, there are several extensive studies of the population genetics of the species, making it possible to sample from the broadest range of genetic variation in the species.

Therefore, we envision a moderate risk to the relocated population through unwanted evolutionary trajectories or carrying unwanted traits to a newly established population. We assess this risk with moderate confidence based on moderate agreement among modest published data. 
$\underline{\text { Section III. Risks of Action to Non-targets within the Recipient Ecosystem }}$

III.A. Risk of Target Transmitting Novel Disease or Associated Pest

There is evidence of an invasive biocontrol weevil pest, Rhinocyllus conicus, ovipositing and developing on Pitcher's thistle (Louda et al. 2005). However, this poses little to no threat to nontargets in the recipient ecosystem, especially when planting Pitcher's thistle seeds. There is no mention of other diseases in the literature available about this species.

Therefore, we judge this risk to be low with moderate confidence based on high agreement among limited available published data on the target.

III.B. Risk of Competitive Interaction Negatively Affecting the Distribution or Abundance of Non-targets

Pitcher's thistle, at a fine geographic scale, rarely co-occurs with two other species of concern (Stellaria longipes and Tanacetum bipinnatum) found in the same Great Lakes dune ecosystems (Marshall 2014). However, because these non-targets naturally occur in lower densities (Marshall 2014), therefore it would be easier to establish Pitcher's thistle well away from other potential sensitive species. Thus, there is little evidence that the relocation of Pitcher's thistle would negatively impact the distribution of these species. Additionally, the large interplant distance in these dunes reduces direct competition.

Therefore, we judge the risk to be low with high confidence based on high agreement among modest published data on the target and other native dune species.

III.C. Risk of Consumptive Effects Reducing the Abundance or Distribution of Non-targets

This risk is not applicable to Pitcher's thistle because it is a vascular plant.

III.D. Risk of Driving Undesired Evolution in Non-targets

Hybridization with nearby common bull thistle (C. vulgare) may be a possibility, though there is no evidence of this happening in areas where they currently occur sympatrically (USFWS 2002).

Therefore, we self-report the risk to be low with moderate confidence based on high agreement and limited evidence.

Overall, we judged risks to non-targets within the recipient ecosystem to be uniformly low given that this is a plant of stressful, mostly open ecosystems where interplant distance is typically large, and plants respond to disturbance.

Section IV. Risks of Action to Non-target, Higher Order Attributes of the Recipient Ecosystem IV.A. Risk of Indirect and Negative Impacts on Ecosystem Structure

A network analysis of a dune plant-pollinator ecosystem revealed that $C$. pitcheri received the most insect visits of all plants in the community, despite their relatively lower abundance (Jolls et al. 2019). Conclusions from this study posed this high pollinator visitation as a benefit to Pitcher's thistle rather than something that results in a negative impact on the ecosystem. 
Therefore, we judge that the risk of indirect and negative impacts on the food web to be low with moderate confidence based on high agreement among limited published data on the target.

\section{IV.B. Risk of Changed Ecosystem Function}

Less is specifically known about how the introduction of this species may impact the recipient ecosystem function. It is possible that Pitcher's thistle may contribute to dune stabilization. However, because the populations, even under the best of circumstances, tend to remain low density and the recipient ecosystem will be a naturally occurring lacustrine dune, we self-report there to be a relatively low risk of strongly adversely impacting recipient ecosystems with moderate confidence.

\section{IV.C. Other Risk}

Exclusion of herbivorous insects can significantly decrease juvenile mortality and increase seed production of Pitcher's thistle (Bevill et al. 1999). If efforts are undertaken by managers to control herbivores during the initial stages of managed relocation, this may negatively affect native insects. However, this impact is likely to be short lived during initial relocation and narrowly focused around planting sites.

Therefore, we judge this additional risk to be low, with high confidence based on modest published data on the target.

Section V. Risks Associated with Biological Invasion

\section{V.A. Risk of Invasion Within the Intended Recipient Ecosystem}

The life history of Cirsium pitcheri, and thistles generally, is as a ruderal. They disperse well, they colonize new habitats. However, despite its role as a colonizer, Pitcher's thistle is often described as a vulnerable species with respect to more invasive species (e.g., Celastrus orbiculatus, oriental bittersweet; Leicht-Young and Pavlovic 2012).

Therefore, we judge the risk of invasion within the recipient location to be moderate with moderate confidence based on modest agreement among limited published data on the target.

\section{V.B. Risk of Invasion Beyond the Recipient Ecosystem}

Experimental dune restorations, restored by planting a common native dune grass, Ammophila breviligulata, did not appear to be colonized by Pitcher's thistle from neighboring dunes (Emery and Rudgers 2010). This may indicate a limited ability of Pitcher's thistle to greatly expand its range through colonization. Although this particular species seems to be relatively poor at population establishment, metapopulation dynamics are cited as essential for the long-term persistence of existing populations. As such, we rate the risk of these species spreading into unknown and unpredictable locations to be moderate. We do not see this species as having a high risk of invading non-dune habitats, and there are not many dune habitats within plausible dispersal distance that aren't part of the shores of these Great Lakes, so we do not judge this risk to be high. Since we have relatively little specific knowledge of the distribution of dunes outside our vaguely defined target area of "dunes toward the northern end of the Great Lakes", we judge our confidence to be moderate. 
Combined, these lead us to judge the risk of invasion beyond the recipient location as low with high confidence based on published data on the target.

\section{V.C. Risk of Irreversibility of the Managed Relocation Action}

Seed banks are very difficult to eliminate. Therefore, if Pitcher's thistle became invasive, we selfreport that the risk that the action cannot be reversed as high with high confidence, also based on modest evidence published on proxy species.

\section{$\underline{\text { Section VI. Risks Associated with Socio-economic Values }}$}

The species has garnered significant research attention, but it is a plant of little obvious social consequence that occupies an ecosystem of little socio-cultural significance, with little evidence that it would degrade ecosystem function.

Therefore, we self-report the risk to societally valued species and ecosystem services to be low with moderate confidence.

\section{Conclusion}

The main risk factors appear to be the potential for the species to spread outside planted areas (Figure $\mathrm{C}-10)$. The species functions as a meta-population with an expectation that local populations come and go. Successful managed relocation would need to assure that this meta-population dynamic is possible and functioning. The dynamic, however, also makes this species harder to remove if it overly succeeds for some reason. This is because the species has light, wind dispersed seeds where individuals are capable of high levels of reproduction. And because the species maintains a longlived seed bank.

The bulk of the evidence from this well-studied species, however, is that populations are hard to get established, and hard to maintain, and that the species generally loses out to other native and nonnative invasive competitors. As a consequence, the risk that the species harms other ecosystems seems moderately low. Since there has been active restoration on-going, we presume that the plant husbandry required to protect extant populations is well enough understood that a managed relocation action would not elevate risk to existing populations.

One source of risk not fully evaluated here is the risk that moving the species could provide a stepping stone for the biocontrol weevil, which infests Pitcher's thistle, to spread to other native thistle species; that moving the species northward provides a habitat bridge for the weevil to other native thistles. We recommend investigating whether that is possible prior to any action. 


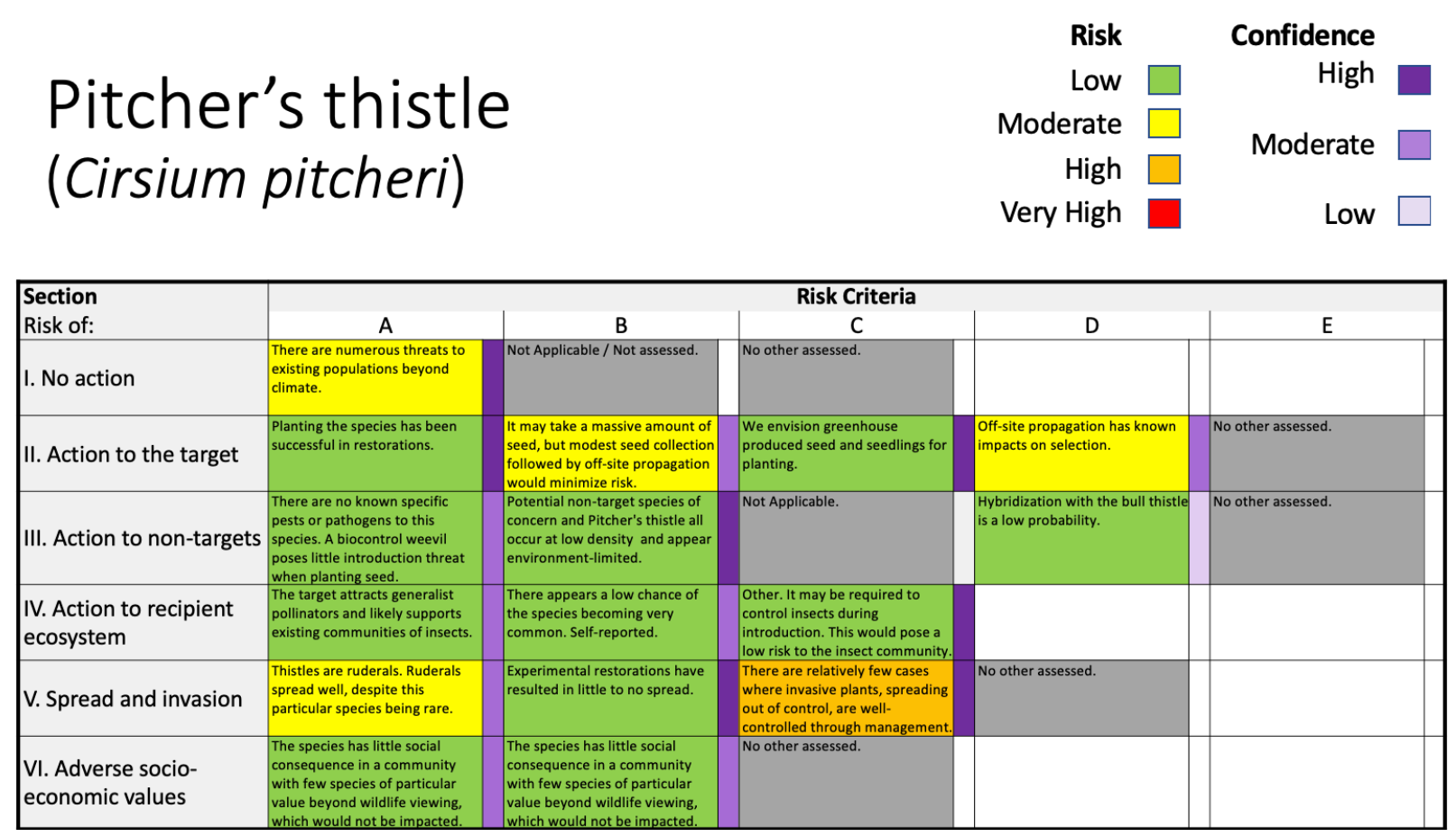

Figure C-10. Risk visualization table of the managed relocation of Pitcher's thistle.

\section{Literature Cited}

Baskett, C. A., S. M. Emery, and J. A. Rudgers. 2011. Pollinator visits to threatened species are restored following invasive plant removal. International Journal of Plant Sciences 172(3):411422.

Bell, T. J., K. I. Powell, and M. L. Bowles. 2013. Viability model choice affects projection accuracy and reintroduction decisions. Journal of Wildlife Management 77:1104-1113.

Bevill, R. L., S. M. Louda, and L. M. Stanforth. 1999. Protection from natural enemies in managing rare plant species. Conservation Biology 13(6):1323-1331.

Bowles, M., R. Flakne, K. McEachern, and N. Pavlovic. 1993. Recovery planning and reintroduction of the federally threatened pitcher's thistle (Cirsium pitcheri) in Illinois. Natural Areas Journal 13:164-176.

Chen, H., and M. A. Maun. 1998. Population ecology of Cirsium pitcheri on Lake Huron sand dunes. III. Mechanisms of seed dormancy. Canadian Journal of Botany-Revue Canadienne De Botanique 76:575-586.

Chen, H., and M. A. Maun. 1999. Effects of sand burial depth on seed germination and seedling emergence of Cirsium pitcheri. Plant Ecology 140:53-60.

D'Ulisse, A., and M. A. Maun. 1996. Population ecology of Cirsium pitcheri on Lake Huron sand dunes. 2. Survivorship of plants. Canadian Journal of Botany-Revue Canadienne De Botanique 74:1701-1707. 
Emery, S. M., P. J. Doran, J. T. Legge, M. Kleitch, and S. Howard. 2013. Aboveground and belowground impacts following removal of the invasive species baby's breath (Gypsophila paniculata) on Lake Michigan sand dunes. Restoration Ecology 21:506-514.

Emery, S. M., and J. A. Rudgers. 2010. Ecological assessment of dune restorations in the Great Lakes region. Restoration Ecology 18(S1):184-194.

Fant, J. B., K. Havens, J. M. Keller, A. Radosavljevic, and E. D. Yates. 2014. The influence of contemporary and historic landscape features on the genetic structure of the sand dune endemic, Cirsium pitcheri (Asteraceae). Heredity 112:519-530.

Fant, J. B., A. Kramer, E. Sirkin, and K. Havens. 2013. Genetics of reintroduced populations of the narrowly endemic thistle, Cirsium pitcheri (Asteraceae). Botany 91:301-308.

Freeland, J. R., J. Gillespie, C. Ciotir, and M. E. Dorken. 2010. Conservation of Hill's thistle (Cirsium hillii). Botany 88:1073-1080.

Gauthier, M., E. Crowe, L. Hawke, N. Emery, P. Wilson, and J. Freeland. 2010. Conservation genetics of Pitcher's thistle (Cirsium pitcheri), an endangered Great Lakes endemic. Botany $88: 250-257$.

Gijmans, F., K. Havens, and P. Vitt. 2020. Effect of capitulum position and weevil infestation on seed production of threatened monocarpic perennial, Cirsium pitcheri. Global Ecology and Conservation 22:e00945.

Girdler, E. B., M. P. Davis, and Z. M. Smith. 2016. Dynamics of an invasion: The spatial interactions of invasive Centaurea stoebe with native Cirsium pitcheri and Tanacetum huronense in a dune environment. American Midland Naturalist 176:20-35.

Girdler, E. B., and T. A. Radtke. 2006. Conservation implications of individual scale spatial pattern in the threatened dune thistle, Cirsium pitcheri. American Midland Naturalist 156:213-228.

Hakes, A. S., and Z. D. Meunier. 2018. Nonhost neighborhood increases biocontrol weevil damage to the nontarget, federally threatened Pitcher's thistle (Cirsium pitcheri). Global Ecology and Conservation 13: e00376.

Halsey, S. J., T. J. Bell, and M. Bowles. 2017a. Initial transplant size and microsite influence transplant survivorship and growth of a threatened dune thistle. Ecological Restoration 35:52-59.

Halsey, S. J., S. Cinel, J. Wilson, T. J. Bell, and M. Bowles. 2017b. Predicting population viability of a monocarpic perennial dune thistle using individual-based models. Ecological Modelling 359:363-371.

Halsey, S. J., T. J. Bell, K. McEachern, and N. B. Pavlovic. 2015. Comparison of reintroduction and enhancement effects on metapopulation viability. Restoration Ecology 23:375-384. 
Halsey, S. J., T. J. Bell, K. McEachern, and N. B. Pavlovic. 2016. Population-specific life histories contribute to metapopulation viability. Ecosphere 7(11):e01536.

Hamze, S. I., and C. L. Jolls. 2000. Germination ecology of a federally threatened endemic thistle, Cirsium pitcheri, of the Great Lakes. American Midland Naturalist 143:141-153.

Havens, K., C. L. Jolls, J. E. Marik, P. Vitt, A. K. McEachern, and D. Kind. 2012. Effects of a nonnative biocontrol weevil, Larinus planus, and other emerging threats on populations of the federally threatened Pitcher's thistle, Cirsium pitcheri. Biological Conservation 155:202-211.

Jolls, C. L., J. N. Inkster, B. G. Scholtens, P. Vitt, and K. Havens. 2019. An endemic plant and the plant-insect visitor network of a dune ecosystem. Global Ecology and Conservation 18:e00603.

Jolls, C. L., J. E. Marik, S. I. Hamze, and K. Havens. 2015. Population viability analysis and the effects of light availability and litter on populations of Cirsium pitcheri, a rare, monocarpic perennial of Great Lakes shorelines. Biological Conservation 187:82-90.

Leicht-Young, S. A., and N. B. Pavlovic. 2012. Encroachment of oriental bittersweet into Pitcher's thistle habitat. Natural Areas Journal 32:171-176.

Louda, S. M., T. A. Rand, A. E. Arnett, A. S. McClay, K. Shea, and A. K. McEachern. 2005a. Evaluation-of ecological risk to populations of a threatened plant from an invasive biocontrol insect. Ecological Applications 15:234-249.

Louda, S. M., T. A. Rand, F. L. Russell, and A. E. Arnett. 2005b. Assessment of ecological risks in weed biocontrol: Input from retrospective ecological analyses. Biological Control 35:253-264.

Marshall, J. M. 2013. Occurrence of Diabrotica undecimpunctata howardi barber (Coleoptera: Chrysomelidae) feeding on Cirsium pitcher flowers. The Great Lakes Entomologist 46(2):138141.

Marshall, J. M. 2014. Influence of topography, bare sand, and soil pH on the occurrence and distribution of plant species in a lacustrine dune ecosystem. Journal of the Torrey Botanical Society 141:29-38.

National Park Service (NPS). 2015. Pitcher's thistle: threatened dune dweller. National Park Service. Great Lakes Research and Education Center, Indiana Dunes National Lakeshore.

Nantel, P. L., J. Jones, and C. Drake. 2018. Viability of multiple populations across the range of a species at risk: The case of Pitcher's thistle, Cirsium pitcheri, in Canada. Global Ecology and Conservation 16:e00445.

Perumal, V. J., and M. A. Maun. 2006. Ecophysiological response of dune species to experimental burial under field and controlled conditions. Plant Ecology 184:89-104. 
Phillips, T., and M. A. Maun. 1996. Population ecology of Cirsium pitcheri on Lake Huron sand dunes .1. Impact of white-tailed deer. Canadian Journal of Botany-Revue Canadienne De Botanique 74:1439-1444.

Promaine, A. 1999. Threatened species monitoring: Results of a 17-year survey of Pitcher's Thistle, Cirsium pitcheri, in Pukaskwa National Park, Ontario. Canadian Field-Naturalist 113:296-298.

Rand, T. A., S. M. Louda, K. M. Bradley, and K. K. Crider. 2015. Effects of invasive knapweed (Centaurea stoebe subsp micranthos) on a threatened native thistle (Cirsium pitcheri) vary with environment and life stage. Botany 93:543-558.

Rowland, J., and M. A. Maun. 2001. Restoration ecology of an endangered plant species: Establishment of new populations of Cirsium pitcheri. Restoration Ecology 9:60-70.

Rudgers, J. A., L. Bell-Dereske, K. M. Crawford, and S. M. Emery. 2015. Fungal symbiont effects on dune plant diversity depend on precipitation. Journal of Ecology 103:219-230.

Staehlin, B. M., and J. B. Fant. 2015. Climate change impacts on seedling establishment for a threatened endemic thistle, Cirsium pitcheri. American Midland Naturalist 173:47-60.

Stanforth, L. M., S. M. Louda, and R. L. Bevill. 1997. Insect herbivory on juveniles of a threatened plant, Cirsium pitcheri, in relation to plant size, density and distribution. Ecoscience 4:57-66.

U.S. Fish and Wildlife Service (USFWS). 2002. Recovery plan for the Pitcher's Thistle (Cirsium pitcheri). Prepared by the Pitcher's thistle recovery team for the US Fish and Wildlife Service. 
The Department of the Interior protects and manages the nation's natural resources and cultural heritage; provides scientific and other information about those resources; and honors its special responsibilities to American Indians, Alaska Natives, and affiliated Island Communities.

NPS 999/175141, March 2021 
Parional Park Service

U.S. Department of the Interior

Natural Resource Stewardship and Science

1201 Oakridge Drive, Suite 150

Fort Collins, CO 80525 